\title{
Renormalization for Golden Circles *
}

\author{
Andreas Stirnemann \\ Department of Mathematics, University of Exeter, Laver Building, North Park Road, \\ Exeter EX4 4QE, UK
}

Received February 18, 1992; in revised form August 10, 1992

\begin{abstract}
We prove that twist maps of the cylinder that are attracted by any fixed point of MacKay's renormalization operator have a transitive invariant golden circle, provided the fixed point satisfies a few simple, purely topological conditions. These conditions can be verified by finite-precision arithmetics; they are fulfilled for the simple fixed point and seem to be fulfilled for the critical fixed point. Taking existence and hyperbolicity of the critical fixed point for granted, we conclude that the standard map has a critical invariant golden circle; the induced map on the circle is topologically conjugate to a rigid rotation; we can show that the conjugator is Hölder continuous; moreover, it is not differentiable on a dense set of points.
\end{abstract}

\section{Contents}

0. Introduction . . . . . . . . . . . . . . . . . . . . . . . . . . . . . . . 369

1. Domain Pairs . . . . . . . . . . . . . . . . . . . . . . . . . . . . 380

2. Fibonacci Strings . . . . . . . . . . . . . . . . . . . . . . . . . . . . . 384

3. Approximate Invariant Sets . . . . . . . . . . . . . . . . . . . . . . . . . 387

4. Asymptotic Self-Similarity . . . . . . . . . . . . . . . . . . . . . . . . . 389

5. The Necklace . . . . . . . . . . . . . . . . . . . . . . . . . . . . . . 396

6. The Golden Curve . . . . . . . . . . . . . . . . . . . . . . . . . . . . . 400

7. Ordering the Patches . . . . . . . . . . . . . . . . . . . . . . . 407

8. Appendix . . . . . . . . . . . . . . . . . . . . . . . . . . . . . . . . . . 422

\section{Introduction}

0.1. Statement of Results. Let us consider one-parameter-families of symmetric areapreserving twist maps of the cylinder. A well known example is the standard family, given by

$$
x^{\prime}=x+y^{\prime}, \quad y^{\prime}=y-\frac{\kappa}{2 \pi} \sin 2 \pi x .
$$

\footnotetext{
* This paper is part of a PhD thesis that is in preparation under the supervision of Oscar E. Lanford III at the ETH. I thank Oscar Lanford for having asked me precisely the right questions
} 

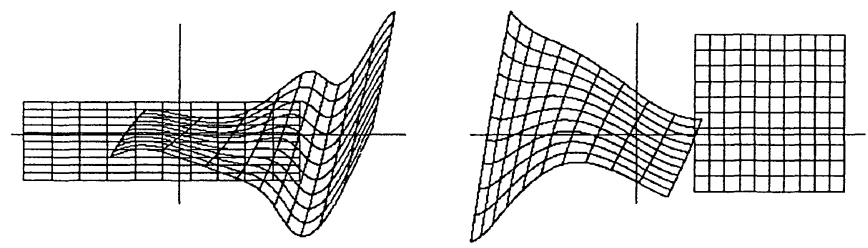

Fig. 1. The action of the critical fixed point

We focus on the question of whether a homotopically non-trivial invariant circle with the golden rotation number exists. For the standard family, there seems to be a critical value $\kappa_{c} \approx 0.9716$ such that below this value there exists a smooth golden circle, while above this value there is none. Our main interest is to find a critical circle, i.e., an invariant curve at precisely the critical parameter value. MacKay has related this problem to the existence of a so-called critical fixed point of a renormalization operator for periodic twist maps (see [2,3]). Numerical evidence strongly suggests that such a fixed point indeed exists; a proof, however, is still lacking. Our aim is to show that, if the critical fixed point exists and satisfies a few simple assumptions, then any map attracted by it has a transitive invariant golden circle. These assumptions are purely topological and can be verified by finite-precision arithmetics. They appear to be fulfilled for the critical fixed point, in the sense that they are satisfied by an approximate fixed point of degree 28 .

If we take existence and hyperbolicity of the critical fixed point for granted, it follows from our theory that any map on its stable manifold has a transitive invariant golden circle. Since the linearization of the operator seems to have exactly one essential expanding eigenvalue at the critical fixed point, the stable manifold has codimension one, and it follows that a generic one-parameter family will intersect it. The critical value mentioned above is interpreted as giving the point of intersection of the standard family with the stable manifold of the critical fixed point. We conclude from our theory that at this parameter value, the standard family has a transitive invariant golden circle.

There is a so-called simple fixed point of the renormalization operator (see [2, Sect.4.3]), given by a linear shear, that seems to be related to the existence of a smooth golden curve. For this fixed point, everything can be calculated explicitly, and it turns out that it satisfies our assumptions too. It follows that any map attracted by the simple fixed point has a transitive invariant golden circle. The argument presented here does not give smoothness of the invariant circle or of the conjugator to the rigid rotation; it only implies that the conjugator is Lipschitz continuous.

We now state the assumptions that make our theory working. Let $D_{U}$ and $D_{T}$ be connected open bounded subsets of the plane. A pair $(U, T)$ of homeomorphisms, defined on $D_{U}$ and $D_{T}$ respectively, is called self-similar if it satisfies the fixed point equation:

$$
U=B T B^{-1}, \quad T=B U T B^{-1} .
$$

Here, $B$ is assumed to be a linear-diagonal map. A self-similar pair will sometimes be called a fixed point. Both the critical and the simple fixed point of MacKay's operator are in this sense self-similar.

Figure 1 shows the action of the critical fixed point on its domains. To the left, we see the sets $D_{T}$ and $T D_{T}$, to the right the sets $U D_{U}$ and $D_{U}$. Roughly speaking, $T$ shifts to the right, and $U$ to the left. 


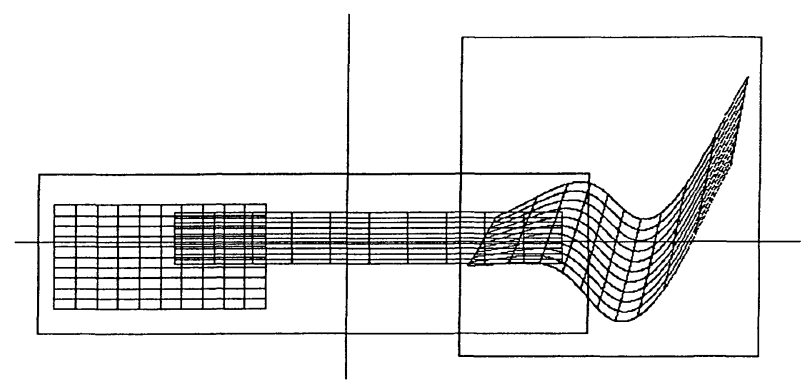

Fig. 2. The domain extension property

A pair of maps $\left(U_{0}, T_{0}\right)$, defined on the domains $D_{U}$ and $D_{T}$ respectively, is called asymptotically self-similar if there exists a sequence of pairs $\left(U_{k}, T_{k}\right)$ (defined on the same domains) and a sequence of linear-diagonal rescalings $B_{k+1}$ such that

$$
\left(U_{k+1}, T_{k+1}\right)=B_{k+1}\left(T_{k}, U_{k} T_{k}\right) B_{k+1}^{-1}, \quad k \geq 0 ;
$$

the maps $U_{k}$ and $T_{k}$ are required to converge uniformly on their domains, and the diagonal elements of $B_{k+1}$ are required to converge. Of course, the limit pair $(U, T)$ is self-similar in the above sense.

Let $F$ be a homeomorphism of the cylinder. Its lift, which will be denoted by the same letter, is a periodic map of the plane; we implement periodicity by requiring that $F$ commute with the backward rotation $R:(x, y) \mapsto(x-1, y)$. The map $F$ is called asymptotically self-similar if the pair $(R, F)$ is asymptotically self-similar.

We now state the basic assumptions on the fixed point $(U, T)$. These assumptions, of course, will not hold for arbitrary domains on which $U$ and $T$ are well defined. But it turns out that we can find special domains, for the critical fixed point as well as for the simple fixed point, such that the assumptions are fulfilled simultaneously. There are six of them. The first three are enough to guarantee existence of a golden circle, but they do not give transitivity.

A1. The diagonal elements of $B$ are of absolute value strictly greater than one.

(This assumption implies that $B^{-1}$ is a contraction. For the critical fixed point, the diagonal elements of $B$ are $\alpha \approx-1.415$ and $\beta \approx-3.067$. For the simple fixed point, we have $\alpha=-\frac{1}{\omega}$ and $\beta=-\frac{1}{\omega^{2}}$, where $\omega=0.618 \ldots$ is the golden number.)

A2. The fixed point has the so called domain extension property (see Sect. 1):

$$
B^{-1} \overline{D_{U}} \subset D_{T}, \quad B^{-1} \overline{D_{T}} \subset D_{T}, \quad T B^{-1} \overline{D_{T}} \subset D_{U} .
$$

(These assumptions play an independent role in renormalization theory: if they hold for complex domains, then the linearization of the renormalization operator (on an appropriate space of analytic pairs) at a fixed point is a compact operator (see [2, Sect. 2.3.8]).)

We illustrate these conditions for the critical fixed point (see Fig. 2). The big rectangle is the set $D_{T}$, the square shaped domain is $D_{U}$. These are said to belong to the zeroth generation. The small patches are (from left to right) $B^{-1} D_{U}, B^{-1} D_{T}$, and $T B^{-1} D_{T}$. (Observe that the rescaling $B$ is a negative matrix.) These are said to belong to the first generation. The conditions imply that the first generation has to be contained in the zeroth one; we see that this is indeed the case for the critical fixed point and the particular domains chosen. 
The third group of assumptions is needed for precluding gaps in the invariant set: A3.

$$
D_{U} \cap D_{T} \neq \emptyset, \quad U D_{T} \cap D_{T} \neq \emptyset, \quad U B^{-1} D_{T} \cap B^{-1} D_{U} \neq \emptyset .
$$

These will be called connectedness assumptions (see Sect. 1). We illustrate them for the critical fixed point (see Fig. 3). The left picture in the first row shows the set $U D_{T}$ and $D_{T}$; the right one shows the sets $D_{T}$ and $D_{U}$. The picture in the second row shows the sets $U B^{-1} D_{T}$ and $B^{-1} D_{U}$.

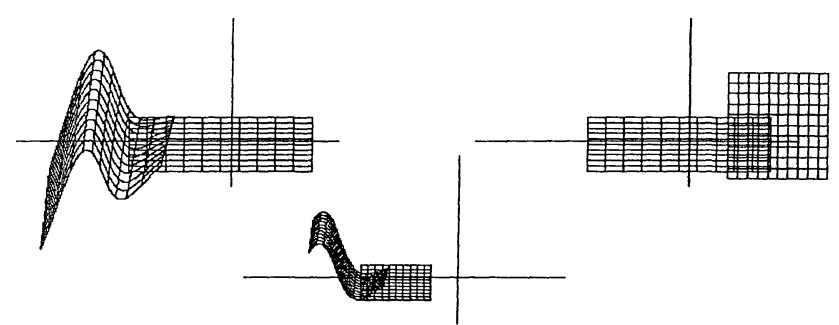

Fig. 3. The connectedness assumptions

Under the assumptions stated so far, we can prove the following result:

Theorem 5.17. If a homeomorphism of the cylinder is attracted by a fixed point that satisfies the assumptions $\mathrm{A} 1, \mathrm{~A} 2$, and $\mathrm{A} 3$, then it has a compact, connected, separating invariant set.

Here, the term separating means that the complement of the invariant set on the cylinder has exactly two unbounded components. If the homeomorphism is an areapreserving, orientation-preserving, end-preserving $C^{1}$ twist diffeomorphism, then by Birhoff's theorem (see $[1,4]$ ), the invariant set is the graph of a Lipschitz function (see Sect.6). This gives the following result:

Theorem 6.9. Let $F$ be an area-preserving, orientation-preserving, end-preserving $C^{1}$ twist diffeomorphism of the cylinder. If $F$ is attracted by any fixed point that satisfies the assumptions $\mathrm{A} 1, \mathrm{~A} 2$, and $\mathrm{A} 3$, then $F$ has an invariant golden circle that is the graph of a Lipschitz function.

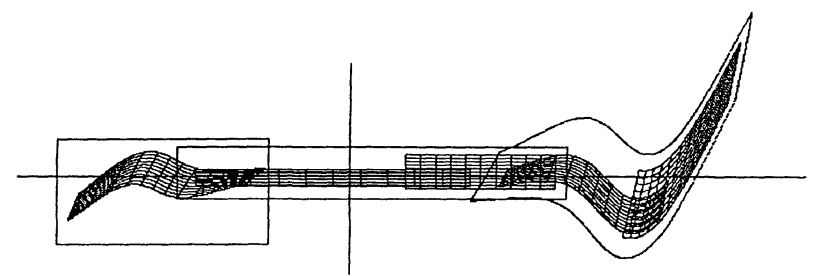

Fig. 4. The first and the second generation

In particular, since the critical fixed point satisfies the assumptions $\mathrm{A} 1, \mathrm{~A} 2$, and $\mathrm{A} 3$, and since the standard family intersects its stable manifold, the standard map has an invariant golden circle at the critical parameter value.

For the idea of the proof, look at Fig. 4. The picture shows the first and the second generation (see Sects. 1 and 7). Observe that the second generation is contained in the 
first one and that its five patches overlap. (These are, from left to right: $U T B^{-2} D_{T}$, $B^{-2} D_{T}, B^{-2} D_{U}, T B^{-2} D_{T}$, and $T B^{-2} D_{U}$.) Moreover, the second generation looks like a thick curve. We shall show that the union of the patches of one generation converges to an invariant curve, with increasing generation.

As mentioned above, these assumptions are not sufficient for transitivity. Transitivity follows if we impose the fourth basic assumptions (see Sect.7):

A4. The map $T B^{-2}$ is a uniform contraction on $D_{U} \cup D_{T}$.

The assumptions of the fifth group will be called disjointness assumptions (see Sect. 7):

A5.

$$
\begin{aligned}
U \overline{D_{T}} \cap \overline{D_{U}} & =\emptyset, \\
B^{-1} \overline{D_{T}} \cap U B^{-1} \overline{D_{T}} & =\emptyset, \\
B^{-1} \overline{D_{T}} \cap U B^{-1} \overline{D_{U}} & =\emptyset, \\
B^{-1} \overline{D_{U}} \cap U B^{-1} \overline{D_{U}} & =\emptyset .
\end{aligned}
$$

We illustrate them for the critical fixed point (see Fig. 5). The picture in the first row shows the sets $U D_{T}$ and $D_{U}$. On the left picture of the second row, we se sets $U B^{-1} D_{U}$ and $B^{-1} D_{U}$. The middle picture shows $U B^{-1} D_{U}$ and $B^{-1} D_{T}$, and the right one shows $U B^{-1} D_{T}$ and $B^{-1} D_{T}$.

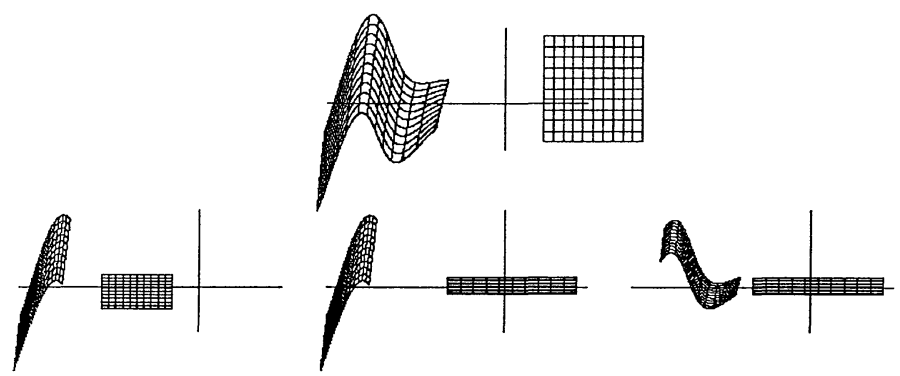

Fig. 5. The disjointness assumptions

Using the disjointness assumptions, we can dispense with the Birkhoff theory in the construction of an invariant curve. This approach, moreover, yields a much more powerful result, namely:

Theorem 7.34. If a homeomorphism of the cylinder is attracted by a fixed point that satisfies the assumptions $\mathrm{A} 1-\mathrm{A} 5$, then it has an invariant golden circle, and the induced mapping on the curve is continuously conjugate to a rigid rotation. In particular, the invariant curve is topologically transitive. Moreover, the conjugator is Hölder continuous. For maps attracted by the critical fixed point, the conjugator is not differentiable on a dense set of points.

The last statement of Theorem 7.34 is implied by the following result:

Lemma 7.32. Under the conditions $\mathrm{A} 1-\mathrm{A} 5$, the number

$$
\mu=\frac{\log |a|}{\log \frac{1}{\omega}}
$$


is an upper bound for the Hölder exponent of the conjugator at the origin. Here, $\alpha$ is the smaller (in absolute value) of the diagonal elements of the rescaling $B$.

For maps attracted by the critical fixed point, $\mu$ is strictly smaller than one, its numerical value being equal to 0.712 . It follows that the conjugator of the critical golden curve is not differentiable at the origin, and, therefore, that it is not differentiable on the orbit of the origin, which is dense. (For maps attracted by the simple fixed point, $\mu$ is equal to one.)

We can show that the Hölder exponent of the conjugator is, in fact, equal to $\mu$; for this, however, we need an additional assumption:

\section{A6. The map $T B^{-1}$ is a uniform contraction on $D_{U} \cup D_{T}$.}

This assumption is stronger than the fourth one. A direct calculation shows that it is satisfied for the simple fixed point. In the case of the critical fixed point, it looks as if it was satisfied too, but the numerical evidence is not conclusive.

Lemma 7.33. Under the conditions $\mathrm{A} 1-\mathrm{A} 6$, the Hölder continuity holds with the number $\mu$ from Lemma 7.32 as exponent.

By Lemma 7.32, this Hölder exponent is optimal.

For maps attracted by the simple fixed point, this lemma implies that the conjugator is Lipschitz continuous.

Now that we have stated all the assumptions, let us indicate how this theory applies to one-parameter families of twist maps and to the critical fixed point. For this purpose, we need a precise definition of the renormalization operator.

0.2. The Three-Step Operator. We shall be dealing only with twist maps of the cylinder, respectively with periodic twist maps (of period one) of the plane. Intuitively speaking, since the rescaling, i.e., conjugation by $B$, destroys this periodicity, we have to renormalize the periodicity too; this leads to the consideration of pairs of maps, the idea being that the initial pair is $(R, F)$, where $R$ is the rigid rotation by -1 , and where $F$ is the periodic twist map. Pairs arising like this from periodic twist maps obviously commute exactly.

We shall be dealing only with symmetric (or reversible) twist maps. Symmetry (or reversibility) means the following. Let $S$ be the reflection across the $y$-axis: $S(x, y)=(-x, y)$. A homeomorphism $F$ of the plane will be called "symmetric" if conjugation by $S$ reverses it:

$$
S F S=F^{-1} \text {. }
$$

Notice that there are (parameter-dependent) coordinate-changes that make the maps of the standard family symmetric in this sense (see [2, Sect. 1.1.4]). The line $\{y=0\}$ will be called the symmetry axis. Symmetry is important in this business for several reasons:

- Symmetry makes it easy to find periodic orbits numerically (see [2, Sect. 1.2.3.1]). (Periodic orbits of certain types are essential for determining the critical parameter value in a given one-parameter family, see [2, Sect.4.4.1].)

- Symmetry is crucial in the very definition of the renormalization operator (see below).

- Restricting to symmetric maps eliminates many unstable directions from the fixed point problem for the renormalization operator.

- The particular symmetry chosen in the renormalization of a one-parameter family of commuting pairs fundamentally affects the result of the iteration process. For instance, 
if we choose the involution $S R$ instead of $S$ in the standard family (at the critical parameter value), we observe convergence to a three-cycle instead of convergence to a fixed point (see [2, Sect. 4.4.1]).

- Symmetric area-preserving maps automatically have zero Calabi invariant (zero flux) (see [2, Sect.4.2.4]); restricting to zero flux is useful, because maps with nonzero flux cannot have invariant curves that go around the cylinder.

On an appropriate space of pairs of symmetric area-preserving maps, MacKay's renormalization operator for the golden number acts as follows (see [2, Sect.4.1.1]):

$$
\tilde{U}=B T B^{-1}, \quad \tilde{T}=B T U B^{-1} .
$$

(Notice that for reasons that will become apparent in Sect.2, we chose a different composition order in the second equation than MacKay; namely $U T$ instead of $T U$.)

This operator has a disadvantage: it breaks the symmetry, unless $U$ and $T$ commute. For the composition $T U$ of two symmetric maps $U$ and $T$ is symmetric if and only if their multiplicative commutator $C=U^{-1} T^{-1} U T$ is equal to the identity:

$$
S T U S T U=S T S S U S T U=T^{-1} U^{-1} T U=C^{-1} .
$$

Since exact commutativity is a very restrictive condition, this disadvantage is a grave one. Although it is not crucial for our purposes, a possible solution of this problem may be of interest. The idea is to iterate the operator three times and to rearrange the composition order such that it becomes palindromical. Since the critical fixed point is commutative, this makes sense. Let us, therefore, consider the following operator:

$$
\tilde{U}=B_{3} T U T B_{3}^{-1}, \quad \tilde{T}=B_{3} T U T U T B_{3}^{-1}, \quad S=B_{3} S B_{3}^{-1} .
$$

Here, the rescaling $B_{3}$ is a product of three single-step rescalings. The third equation expresses the fact that the rescaling $B_{3}$ preserves the symmetry.

We shall call this operator the three-step operator and denote it by $N$. The threestep operator has the following properties:

- $N$ preserves symmetry.

- $N$ preserves area-preservation.

- $N$ preserves commutativity.

- A commuting fixed point of $N$ is a fixed point or a three-cycle for MacKay's operator.

0.3. The Normalization Conditions. In order to have a well defined renormalization operator, we need a prescription for calculating the rescaling $B_{3}$ for a given pair $(U, T)$. We shall do this by giving two normalization conditions that determine the rescaling $B_{3}$ by the requirement that the pair $B_{3}(T U T, T U T U T) B_{3}^{-1}$ be normalized. A possible choice is the following: a pair $(U, T)$ is called normalized if the map $T$ has a fixed point at a prescribed location on the symmetry axis, e.g. at $(0,-1)$, and if $U$ maps this point by one unit to the left: $\pi_{1} U(0,-1)=-1$. (Here, $\pi_{1}$ is the projection onto the first coordinate direction.) It can be shown that the set of normalizable pairs is open in an appropriate space of symmetric pairs. (A fixed point of a symmetric map $T$ on the symmetry axis can be interpreted as the point of intersection of a particular curve with the symmetry axis. Namely as the point where the symmetry line of the involution $S T$ intersects the symmetry axis. (For the notation of symmetry lines, see [2, Sect. 1.1.4].) For symmetric twist maps, this intersection can be shown to be transversal. Numerical evidence suggests that the operator can be iterated many times in a neighborhood of the pair $(R, F)$, where $F$ is the standard map at the critical parameter value. 
0.4. The Critical Fixed Point. It is well known that MacKay's operator has a critical fixed point that is area-preserving and commuting. (These latter claims follow from the fact that there are area-preserving commuting pairs attracted by the critical fixed point.) (Note that there may be non-trivial fixed points different from MacKay's; in this paper, the term critical fixed point always refers to the one found by MacKay.)

Furthermore, it is well known that, besides the critical fixed point, MacKay's operator has a critical three-cycle (see [2, Sect. 4.4.1]). We expect, therefore, that the three-step operator has (at least) four non-trivial fixed points, three of which correspond to the critical three-cycle. This is indeed the case. The non-trivial fixed point of the three-step operator that is a fixed point for MacKay's operator too will be called the dominant fixed point. Let us denote it by $(U, T)$. Then we have

$$
U=B_{3} T U T B_{3}^{-1}, \quad T=B_{3} T U T U T B_{3}^{-1}, \quad S=B_{3} S B_{3}^{-1},
$$

where $B_{3}$ is the third power of the critical single-step rescaling $B$. We show that existence of additional (so-called subdominant) fixed points can be deduced from this equation; these correspond to the critical three-cycle of MacKay's operator. Consider, for instance, the involution $S U$. Since $U$ and $T$ commute, $S U$ is a symmetry for both $U$ and $T$. Using the first and the third of the above equations, we write

$$
\begin{aligned}
S U & =S B_{3} T U T B_{3}^{-1}=B_{3} S T U T B_{3}^{-1}=B_{3} T^{-1} S U T B_{3}^{-1} \\
& =B_{3} T^{-1} S U\left(B_{3} T^{-1}\right)^{-1} .
\end{aligned}
$$

Since $T$ (and therefore $T^{-1}$ as well) commutes with $U$, we may rewrite the fixed point equation as follows:

$$
\begin{aligned}
U & =B_{3} T^{-1} \operatorname{TUT}\left(B_{3} T^{-1}\right)^{-1}, \\
T & =B_{3} T^{-1} \operatorname{TUTUT}\left(B_{3} T^{-1}\right)^{-1}, \\
S U & =B_{3} T^{-1} S U\left(B_{3} T^{-1}\right)^{-1} .
\end{aligned}
$$

This fixed point equation corresponds to an operator that has the same temporal part (i.e., that involves the same compositions) as the old one, but that preserves the symmetry $S U$ instead of the standard symmetry. It is this operator, therefore, that governs the critical behavior observed for instance in the standard family with the involution $S R$. If we choose coordinates such that $B_{3} T^{-1}$ is linear-diagonal, we obtain one of the three universal pairs forming the three-cycle, and we see that this one is just a coordinate transform of the dominant fixed point, a fact conjectured by MacKay (see [2, Sect.4.4.2, p. 4.4.42]).

The fixed point of the rescaling $B_{3} T^{-1}$ is the point of intersection of the golden curve with the symmetry line of the involution $S U$, because the map $B_{3} T^{-1}$ has the golden curve as well as this symmetry line as invariant curves. The eigenvalues of the linearization $D\left(B_{3} T^{-1}\right)$ at the fixed point are the so-called subdominant scaling factors $\alpha_{s} \approx-4.85$ and $\beta_{s} \approx-16.86$. It is a numerical fact that they are different from the third powers of the dominant single-step scaling factors $\alpha$ and $\beta$. But there is a simple relation between the dominant and the subdominant scaling factors, which can be derived as follows:

$$
\alpha_{s} \beta_{s}=\operatorname{det}\left(D\left(B_{3} T^{-1}\right)\right)=\operatorname{det}\left(B_{3}\right) \operatorname{det}\left(D T^{-1}\right)=\operatorname{det}\left(B_{3}\right)=\operatorname{det}(B)^{3}=(\alpha \beta)^{3} .
$$

(Here, the Jacobian is to be taken at the fixed point of $B_{3} T^{-1}$.) This identity explains a well known experimental fact (see [5]). 
0.5. The Spectrum at the Dominant Fixed Point. We shall concentrate on the dominant fixed point, because the subdominant ones add nothing essentially new. Let us discuss the spectrum of the linearization of the three-step operator at this fixed point. Numerical evidence suggests that there is exactly one essential expanding eigenvalue $\delta^{3} \approx 1.628^{3}$; this one plays the role of Feigenbaum's constant. Furthermore, two parts of the spectrum can be analyzed explicitly: the so-called coordinate-change spectrum and the non-commuting spectrum see [2, Sects. 2.3 .4 and 4.2.4). (The coordinate-change eigenvalues belong to perturbations generated by infinitesimal coordinate-changes. The non-commuting eigenvalues belong to perturbations breaking commutativity.)

It turns out that the coordinate-change spectrum of $D N$ in the space of symmetric perturbations looks as follows:

$$
\alpha_{3}^{-2 k} \beta_{3}^{-l}, \quad \alpha_{3}^{-2 m} \beta_{3}^{1-n}, \quad(k, l, m, n) \in \mathbb{N}_{0}^{4}, \quad(k, l) \neq(0,0), \quad(m, n) \neq(0,1),
$$

where $\alpha_{3}=\alpha^{3}$ and $\beta_{3}=\beta^{3}$. (This is shown in the author's thesis [6]; the calculation is the same as the corresponding one in [2].)

The only expanding values are $\beta_{3}$ (corresponding to a shift along the symmetry axis) and $\beta_{3} / \alpha_{3}^{2}$, corresponding to a perturbation $\Gamma(x, y)=\left(0, x^{2}\right)$, which generates a quadratic shear. For the dominant fixed point (with scaling factors $\alpha_{3} \approx-2.838$ and $\beta_{3} \approx-28.65$ ), the absolute value of $\beta_{3} / \alpha_{3}^{2}$ happens to be smaller than one.

It turns out that the non-commuting spectrum looks as follows:

$$
-\alpha_{s}^{-2 k} \beta_{s}^{-l}, \quad-\alpha_{s}^{-2 m} \beta_{s}^{1-n}, \quad(k, l, m, n) \in\left(\mathbb{N}_{0}\right)^{4} .
$$

Here, $\alpha_{s}$ and $\beta_{s}$ are the subdominant scaling factors introduced above. (This is shown in the author's thesis [6]; the calculation is similar in spirit to the corresponding one in [2]).

We see that the non-commuting spectrum, apart from the two eigenvalues equal to -1 , is the negative of the coordinate-change spectrum, with the subdominant scaling factors replacing the dominant ones. (Compare this with MacKay's operator, where the non-commuting spectrum is the exact negative of the coordinate-change spectrum (see [2, Sect. 4.2.4]).)

We are going to work with pairs that stem from symmetric periodic twist maps. Then the non-commuting part of the spectrum does not turn up. But the coordinatechange eigenvalues certainly do, and we have seen that there are two expanding ones. In general, these will make the iteration process divergent. We can cope with this problem in two ways. Either we work with quadratic rescalings, obtained by composing a linear-diagonal rescaling with a quadratic symmetry-preserving shear of the form

$$
(x, y) \mapsto\left(x,-c+y+Q x^{2}\right) .
$$

(For the details, see the next paragraph. In order to determine the two additional coefficients $c$ and $Q$, we need two more normalization conditions.) Then we argue that the whole theory to be developed in the next sections works just as well with quadratic rescalings. (In particular, it is not necessary for our theory that different rescalings commute.) Or we can argue as follows: the presence of two unstable coordinate-change eigenvalues implies that the stable manifold of the fixed point has codimension 3; therefore, generic three-parameter families can be expected to intersect it. Now we simply make the standard family a three-parameter family, by conjugating it with the above quadratic shears. Numerical experience suggests that there is a quadratic shear such that the standard family, conjugated by it, intersects 
the stable manifold of the dominant fixed point. (The coordinates given by this shear are called scaling coordinates by MacKay, see [2, Sect. 2.3.5]; scaling coordinates are coordinates in which the linear-diagonal renormalization converges; we shall clarify this concept in the next paragraph.)

One last remark: for combinatorial reasons, the three-step operator is not convenient for our theory. But it is easy to see that a twist map that is asymptotically self-similar with respect to the three-step operator and the dominant fixed point is asymptotically self-similar with respect to the single-step operator too. Therefore, it is asymptotically self-similar in the sense stated at the beginning of this section.

Summing up: Nothing prevents us from applying our theory to generic oneparameter families of symmetric twist maps on the cylinder, and to the critical fixed point.

0.6. Scaling Coordinates. In the last paragraph, we showed by an abstract argument that there are coordinates, given by a quadratic shear, such that, at the critical value, the linear-diagonal renormalization yields convergence to the critical fixed point. Here, we are going to construct these coordinates explicitly. I suggest that this paragraph be skipped on first reading.

For simplicity, we consider the single-step operator:

$$
\tilde{U}=B T B^{-1}, \quad \tilde{T}=B U T B^{-1} .
$$

As mentioned before, we have to use quadratic rescalings in order to make the renormalization process at the critical value convergent. Let us write $B$ in the following form:

$$
x^{\prime}=\alpha x, \quad y^{\prime}=\beta\left(-c+y+Q x^{2}\right) .
$$

(The number $c$ can be thought of as (an approximation to) the $y$-coordinate of the point of intersection of the golden curve with the symmetry line $\{x=0\}$.)

The rescaling $B$ is obtained by composing a quadratic, symmetry-preserving shear

$$
\Gamma:(x, y) \mapsto\left(x,-c+y+Q x^{2}\right)
$$

with a linear-diagonal scale change

$$
\Delta:(x, y) \mapsto(\alpha x, \beta y) ;
$$

i.e., we have

$$
B=\Delta \Gamma .
$$

Note that conjugation by $B$ preserves symmetry. Moreover, it preserves areapreservation, the Jacobian of $B$ being constant.

The rescaling $B$ can be defined by enforcing certain normalization conditions. (Of course, we need four of them.) Their precise nature is not important for our present purposes.

The stable manifold at the critical fixed point of the operator thus defined has codimension 1 (in the space of commuting pairs). Let $\left(U_{0}, T_{0}\right)$ be a commuting pair on this stable manifold. We shall use the notation

$$
F_{j}=T_{0}^{q_{\jmath}} U_{0}^{p_{\imath}}
$$

where $\left(p_{j}, q_{j}\right)$ denotes the $j^{\text {th }}$ Fibonacci pair; the sequence of Fibonacci pairs, starting with $j=-1$, looks as follows:

$$
(1,0),(0,1),(1,1),(1,2),(2,3),(3,5), \ldots
$$


Iterating the operator $j$ times, with starting point $\left(U_{0}, T_{0}\right)$, generates the pair

$$
\left(U_{j}, T_{j}\right)=B_{j} \ldots B_{1}\left(F_{j-1}, F_{j}\right) B_{1}^{-1} \ldots B_{j}^{-1} .
$$

Since, by assumption, $\left(U_{0}, T_{0}\right)$ lies on the stable manifold of the fixed point, the pairs $\left(U_{j}, T_{j}\right)$ converge. Moreover, the rescalings $B_{j}$ converge (in the sense that the sequences $\alpha_{j}, \beta_{j}, c_{j}$, and $Q_{j}$ converge).

The limits $\alpha_{\infty}=\lim \alpha_{j}$ and $\beta_{\infty}=\lim \beta_{j}$ are simply the scaling factors at the critical fixed point, which we denoted by $\alpha$ and $\beta$ in Sect.0.1. (The limits of the sequences $c_{j}$ and $Q_{j}$ depend on the normalization conditions; they are not important here.)

We shall need the following numerical facts:

$$
\left|\frac{1}{\beta_{\infty}}\right|<1, \quad\left|\frac{\alpha_{\infty}^{2}}{\beta_{\infty}}\right|<1 .
$$

(Remember that we have $\alpha_{\infty} \approx-1.415$ and $\beta_{\infty} \approx-3.067$.)

In the same way as before, we write $B_{j}=\Delta_{j} \Gamma_{j}$. In this notation, we have

$$
\left(U_{j}, T_{j}\right)=\Delta_{\jmath} \Gamma_{j} \ldots \Delta_{1} \Gamma_{1}\left(F_{\jmath-1}, F_{j}\right) \Gamma_{1}^{-1} \Delta_{1}^{-1} \ldots \Gamma_{j}^{-1} \Delta_{j}^{-1} .
$$

Now we make two simple observations. First: any two of the above quadratic shears commute: $\Gamma_{j} \Gamma_{k}=\Gamma_{k} \Gamma_{j}$. Moreover, the composition is a shear of the same kind, given by $c=c_{j}+c_{k}$ and $Q=Q_{j}+Q_{k}$. Therefore, the quadratic shears can be identified with $\mathbb{R}^{2}$, composition corresponding to vector addition.

Second: commuting a quadratic shear $\Gamma$ through a linear-diagonal scale change $\Delta$ acts on $\Gamma$ as follows:

$$
\begin{gathered}
\Gamma \Delta=\Delta \tilde{\Gamma} \Rightarrow \tilde{\Gamma}=\left(\begin{array}{cc}
\frac{1}{\beta} & 0 \\
0 & \frac{\alpha^{2}}{\beta}
\end{array}\right) \Gamma, \\
\Delta \Gamma=\hat{\Gamma} \Delta \Rightarrow \hat{\Gamma}=\left(\begin{array}{cc}
\beta & 0 \\
0 & \frac{\beta}{\alpha^{2}}
\end{array}\right) \Gamma .
\end{gathered}
$$

(From now on, we regard the shears as two-dimensional vectors; the first equation, e.g., means that the number $c$ is multiplied by $\frac{1}{\beta}$ and that the number $Q$ is multiplied by $\frac{\alpha^{2}}{\beta}$.)

In the expression for $\left(U_{j}, T_{j}\right)$, we commute the shears $\Gamma_{j}$ to the center. According to the above observations, we obtain

where

$$
\left(U_{j}, T_{j}\right)=\Delta_{j} \ldots \Delta_{1} \tilde{\Gamma}_{j}\left(F_{j-1}, F_{j}\right) \tilde{\Gamma}_{\jmath}^{-1} \Delta_{1}^{-1} \ldots \Delta_{\jmath}^{-1}
$$

$$
\tilde{\Gamma}_{j}=\Gamma_{1}+\left(\begin{array}{cc}
\frac{1}{\beta_{1}} & 0 \\
0 & \frac{\alpha_{1}^{2}}{\beta_{1}}
\end{array}\right) \Gamma_{2}+\ldots+\left(\begin{array}{cc}
\frac{1}{\beta_{1} \ldots \beta_{j-1}} & 0 \\
0 & \frac{\alpha_{1}^{2} \ldots \alpha_{j-1}^{2}}{\beta_{1} \ldots \beta_{j-1}}
\end{array}\right) \Gamma_{j} .
$$


The maps $\tilde{\Gamma}_{j}$ converge, since the resulting series is dominated by a convergent geometric series. (Here, we are using the convergence of the series $\alpha_{j}$ and $\beta_{j}$, the above numerical facts, and the fact that the maps $\Gamma_{j}$ are bounded (they even converge).) Let $\tilde{\Gamma}$ denote the limit. We have

$$
\tilde{\Gamma}=\Gamma_{1}+\left(\begin{array}{cc}
\frac{1}{\beta_{1}} & 0 \\
0 & \frac{\alpha_{1}^{2}}{\beta_{1}}
\end{array}\right) \Gamma_{2}+\left(\begin{array}{cc}
\frac{1}{\beta_{1} \beta_{2}} & 0 \\
0 & \frac{\alpha_{1}^{2} \alpha_{2}^{2}}{\beta_{1} \beta_{2}}
\end{array}\right) \Gamma_{3}+\ldots
$$

Now, we replace $\tilde{\Gamma}_{j}$ by $\tilde{\Gamma}$ in the expression for $\left(U_{\jmath}, T_{\jmath}\right)$ and claim that the resulting pairs

$$
\Delta_{j} \ldots \Delta_{1} \tilde{\Gamma}\left(F_{\jmath-1}, F_{j}\right) \tilde{\Gamma}^{-1} \Delta_{1}^{-1} \ldots \Delta_{j}^{-1}
$$

still converge. In order to prove this, we commute the error term $\tilde{\Gamma}_{j}-\tilde{\Gamma}$ all the way through to the left, and its inverse to the right. Because of

$\tilde{\Gamma}_{\jmath}-\tilde{\Gamma}=-\left(\begin{array}{cc}\frac{1}{\beta_{1} \ldots \beta_{\jmath}} & 0 \\ 0 & \frac{\alpha_{1}^{2} \ldots \alpha_{j}^{2}}{\beta_{1} \ldots \beta_{j}}\end{array}\right) \Gamma_{\jmath+1}-\left(\begin{array}{cc}\frac{1}{\beta_{1} \ldots \beta_{\jmath+1}} & 0 \\ 0 & \frac{\alpha_{1}^{2} \ldots \alpha_{\jmath+1}^{2}}{\beta_{1} \ldots \beta_{j+1}}\end{array}\right) \Gamma_{\jmath+2}-\ldots$,

the error term commuted through becomes

$$
-\Gamma_{j+1}-\left(\begin{array}{cc}
\frac{1}{\beta_{j+1}} & 0 \\
0 & \frac{\alpha_{j+1}^{2}}{\beta_{j+1}}
\end{array}\right) \Gamma_{\jmath+2}-\ldots
$$

By dominated convergence, these maps converge as $j \rightarrow \infty$, and we have proved that the modified sequence $(*)$ converges. (Here, we are using the convergence of the sequences $\alpha_{j}$ and $\beta_{j}$, the above numerical facts, and the fact that the maps $\Gamma_{\jmath}$ converge. $)$ But this is the same as saying that the pair $\tilde{\Gamma}\left(U_{0}, T_{0}\right) \tilde{\Gamma}^{-1}$ is asymptotically self-similar. The scaling coordinates, therefore, are those given by the map $\tilde{\Gamma}$.

\section{Domain Pairs}

We consider an arbitrary fixed point of the renormalization operator for twist maps. The aim of the first three sections is to construct a compact connected invariant pair of sets for this fixed point. In the first section, we recursively define a sequence of pairs of sets and show that the limit pair is compact and connected. In the second section, we give an explicit representation of these pairs, which is used in the third section to show that the limit pair is invariant for the fixed point in a sense that will be defined there. The assumptions on the fixed point are stated as they are needed.

Let $D_{U}$ and $D_{T}$ be connected open bounded subsets of the plane, and let $(U, T)$ be a pair of homeomorphisms defined on $D_{U}$ and $D_{T}$ respectively.

We assume that the pair $(U, T)$ satisfies the fixed point equation

$$
U=B T B^{-1}, \quad T=B U T B^{-1},
$$


where $B$ is a linear-diagonal map. The only additional assumption on $B$ we shall need later on is the following:

A1. The diagonal elements of $B$ are of absolute value strictly greater than one.

(For the present purposes we need not, and do not, assume that $U$ and $T$ commute. Therefore, the composition order in the second equation is significant.)

The second basic assumption is that the maps on the right-hand sides of the fixed point equation are defined on domains that are strictly larger than the domains of $U$ and $T$ respectively. This property will be called domain extension property. Specifically, we require

A2.

$$
B^{-1} \overline{D_{U}} \subset D_{T}, \quad B^{-1} \overline{D_{T}} \subset D_{T}, \quad T B^{-1} \overline{D_{T}} \subset D_{U}
$$

In particular, it follows from this that $T D_{T}$ and $U D_{U}$ are bounded sets. Moreover, it follows that the equation $U=B T B^{-1}$ extends $U$ to a homeomorphism on $D_{U} \cup D_{T}$. From now on, we take $U$ to be this extension, i.e., we assume that $U$ is defined on $D_{T}$ as well.

Let us now define a sequence of pairs $\left(M_{j}, N_{\jmath}\right)$ of open sets that starts with the pair $\left(D_{U}, D_{T}\right)$; the terms of this sequence will be called domain pairs.

We put $M_{0}=D_{U}, N_{0}=D_{T}$ and

$$
M_{\jmath+1}=T B^{-1} N_{\jmath}, \quad N_{j+1}=B^{-1}\left(M_{j} \cup N_{j}\right)
$$

for $j \geq 0$, so long as $T B^{-1}$ is well defined on $N_{j}$.

Lemma 1.1. Under the assumption $\mathrm{A} 2$, the pairs $\left(M_{j}, N_{j}\right)$ are well defined for all non-negative integers $j$, and the following relations hold:

$$
\overline{M_{j+1}} \subset M_{\jmath}, \quad \overline{N_{\jmath+1}} \subset N_{j}
$$

for all non-negative integers $j$.

Proof. We prove the claims

$$
T B^{-1} \overline{N_{j}} \subset M_{j}, \quad B^{-1} \overline{M_{j}} \subset N_{j}, \quad B^{-1} \overline{N_{j}} \subset N_{\jmath}
$$

by induction. For $j=0$, they are identical to the domain extension property. We have $B^{-1} \overline{N_{0}}=B^{-1} \overline{D_{T}} \subset D_{T}$; therefore, $M_{1}$ is well defined. ( $N_{1}$ is well defined anyway.)

Let $j$ be greater than or equal to zero; we assume that the claims have been proved up to $j$ and that $M_{j+1}$ and $N_{\jmath+1}$ are well defined. Then we have

$$
T B^{-1} \overline{N_{j+1}}=T B^{-1} B^{-1} \overline{M_{j}} \cup T B^{-1} B^{-1} \overline{N_{j}} \subset T B^{-1} N_{\jmath}=M_{j+1}
$$

by the definitions of $N_{j+1}$ and of $M_{j+1}$ and by the inductive assumption. In the same way we obtain

$$
B^{-1} \overline{M_{\jmath+1}}=B^{-1} T B^{-1} \overline{N_{\jmath}} \subset B^{-1} M_{j} \subset N_{\jmath+1}
$$

and

$$
B^{-1} \overline{N_{\jmath+1}}=B^{-2} \overline{M_{j}} \cup B^{-2} \overline{N_{\jmath}} \subset B^{-1} N_{j} \subset N_{\jmath+1}
$$


In particular, it follows that we have

$$
B^{-1} \overline{N_{\jmath+1}} \subset B^{-1} N_{\jmath} \subset B^{-1} N_{0}=B^{-1} D_{T} \subset D_{T},
$$

which shows that $M_{j+2}$ is well defined.

[For this proof, the fixed point equation is not needed.]

We have shown that the sets $\bar{M}_{j}$ and $\overline{N_{j}}$ form decreasing sequences of non-empty compact sets. Next, we impose an additional assumption on the domains $D_{U}$ and $D_{T}$, namely

A3.

$$
D_{U} \cap D_{T} \neq \emptyset, \quad U D_{T} \cap D_{T} \neq \emptyset, \quad U B^{-1} D_{T} \cap B^{-1} D_{U} \neq \emptyset .
$$

(Remember that $U$ is defined on $D_{T}$ as well.) These assumptions will be called connectedness assumptions.

Lemma 1.2. Under the assumptions $\mathrm{A} 2$ and $\mathrm{A} 3$, the following relations hold:

$$
U N_{\jmath} \cap N_{\jmath} \neq \emptyset, \quad U B^{-1} N_{\jmath} \cap B^{-1} M_{j} \neq \emptyset
$$

for all non-negative integers $j$.

Proof. For $j=0$, these claims correspond to the second and third connectedness assumption. Proceeding by induction, we obtain

$$
\begin{aligned}
U N_{j+1} \cap N_{j+1} & =U\left(B^{-1} M_{j} \cup B^{-1} N_{j}\right) \cap\left(B^{-1} M_{\jmath} \cup B^{-1} N_{\jmath}\right) \\
& \supset U B^{-1} N_{\jmath} \cap B^{-1} M_{\jmath} \neq \emptyset .
\end{aligned}
$$

Making use of the fixed point equation, we obtain furthermore

$$
\begin{aligned}
U B^{-1} N_{\jmath+1} \cap B^{-1} M_{\jmath+1} & =U B^{-2}\left(M_{\jmath} \cup N_{\jmath}\right) \cap B^{-1} T B^{-1} N_{\jmath} \\
& =U B^{-2}\left(M_{j} \cup N_{\jmath}\right) \cap B^{-1} B U T B^{-2} N_{\jmath} \\
& \supset U\left(B^{-2} M_{\jmath} \cap T B^{-2} N_{\jmath}\right) \\
& =U\left(B^{-2} M_{\jmath} \cap B^{-1} B T B^{-1} B^{-1} N_{j}\right) \\
& =U B^{-1}\left(B^{-1} M_{\jmath} \cap U B^{-1} N_{j}\right) .
\end{aligned}
$$

The last set on the right-hand side is not empty by the inductive assumption.

Lemma 1.3. Under the assumptions $\mathrm{A} 2$ and $\mathrm{A} 3$, we have

$$
M_{j} \cap N_{j} \neq \emptyset
$$

for all non-negative integers $j$.

Proof. For $j=0$, the claim amounts to the relation $D_{U} \cap D_{T} \neq \emptyset$, which is true by the first connectedness assumption.

We have $M_{j+1}=T B^{-1} N_{j}=B^{-1} U N_{j}$ by the fixed point equation. (Remember that we assume $U$ to be defined on $D_{T}$ too, and that $N_{j} \subset D_{T}$ by Lemma 1.1.) Since $N_{j+1}=B^{-1} M_{j} \cup B^{-1} N_{j}$, we obtain

$$
N_{\jmath+1} \cap M_{j+1} \supset B^{-1} N_{j} \cap B^{-1} U N_{j}=B^{-1}\left(N_{j} \cap U N_{j}\right) \neq \emptyset
$$

by Lemma 1.2 . 
Lemma 1.4. Under the assumptions $\mathrm{A} 2$ and $\mathrm{A} 3$, the sets $M_{\jmath}$ and $N_{\jmath}$ are connected for all non-negative integers $j$.

Proof. $M_{0}=D_{U}$ and $N_{0}=D_{T}$ are connected by assumption. Assuming that $M_{j}$ and $N_{j}$ are connected, so is $M_{\jmath+1}$, because it is the continuous image of a connected set, and so is $N_{j+1}$, because it is the continuous image of an overlapping union of two connected sets.

Now, we define the sets $L_{j}$ by $L_{\jmath}=M_{j} \cup N_{\jmath}$.

Lemma 1.5. The sets $L_{\jmath}$ satisfy the following recursion relation:

$$
L_{j+2}=B^{-1} L_{j+1} \cup T B^{-2} L_{\jmath}
$$

for all non-negative integers $j$.

Proof. By definition, we have

$$
\begin{aligned}
L_{j+2} & =M_{j+2} \cup N_{\jmath+2} \\
& =T B^{-1} N_{j+1} \cup B^{-1}\left(M_{j+1} \cup N_{\jmath+1}\right) \\
& =T B^{-1} B^{-1}\left(N_{\jmath} \cup M_{j}\right) \cup B^{-1} L_{j+1} \\
& =T B^{-2} L_{\jmath} \cup B^{-1} L_{\jmath+1}
\end{aligned}
$$

for $j \geq 0$.

Finally, we take the limit pair of the $\left(M_{j}, N_{\jmath}\right)$ :

$$
(M, N)=\left(\bigcap_{j} M_{\jmath}, \bigcap_{\jmath} N_{j}\right) .
$$

The union of $M$ and $N$ will be denoted by $L$. We have

$$
L=M \cup N=\bigcap_{\jmath}\left(M_{\jmath} \cup N_{j}\right)=\bigcap_{j} L_{j} .
$$

(Remember that the sequences $\left(M_{\jmath}\right)$ and $\left(N_{j}\right)$ are decreasing.)

We call a pair of sets non-empty, respectively compact, respectively connected, if the union of its two sets is non-empty, respectively compact, respectively connected.

Lemma 1.6. Under the asumptions $\mathrm{A} 2$ and $\mathrm{A} 3$, the limit pair $(M, N)$ thus defined is non-empty, compact, and connected.

Proof. Since $\overline{L_{j+1}} \subset L_{\jmath} \subset \overline{L_{j}}$, it follows that we have

$$
\bigcap_{j} L_{j}=\bigcap_{\jmath} \overline{L_{j}}
$$

The assertions now follow from the fact that $L$ is the intersection of a decreasing family of non-empty compact connected sets. 


\section{Fibonacci Strings}

We are going to show that the sets $L$, that we constructed recursively in the previous section are in a certain sense approximately invariant for the pair $(U, T)$. The principal tool will be an explicit expression for these sets. This expression will involve the notion of Fibonacci strings, which we are going to define first.

Consider the fixed point equation

$$
U=B T B^{-1}, \quad T=B U T B^{-1} .
$$

Iterating it once yields

$$
U=B^{2} U T B^{-2}, \quad T=B^{2} T U T B^{-2},
$$

and once more

$$
U=B^{3} T U T B^{-3}, \quad T=B^{3} U T T U T B^{-3} .
$$

The sequences of the two symbols $U$ and $T$ arising like this on the right-hand sides are called Fibonacci strings; we denote them by $F_{j}$. Formally, they are defined as follows:

$$
\begin{gathered}
F_{-1}=U, \quad F_{0}=T, \\
F_{j+2}=F_{\jmath} F_{\jmath+1}, \quad j \geq-1 .
\end{gathered}
$$

We shall sometimes interpret these strings as mappings, namely as the corresponding compositions of the maps $U$ and $T$. Note that the composition order $F_{j} F_{j+1}$, rather than $F_{j+1} F_{j}$, implies that the strings grow to the left: for any two Fibonacci strings with $j \geq 0$, the shorter one is a tail of the longer one. (This property was the objective for using the composition $U T$ rather than $T U$ in the fixed point equation; it is crucial for the definition of the Fibonacci tails (see below).)

Lemma 2.1. The fixed point equation translates into

$$
F_{j}=B F_{j+1} B^{-1}, \quad j \geq-1 .
$$

Proof. For $j=-1$ and $j=0$, the assertions correspond to the fixed point equation. Proceeding by induction, we obtain

$$
F_{j+1}=F_{\jmath-1} F_{\jmath}=B F_{\jmath} B^{-1} B F_{\jmath+1} B^{-1}=B F_{\jmath} F_{j+1} B^{-1}=B F_{j+2} B^{-1}
$$

for $j \geq 0$.

Lemma 2.2.

$$
\begin{aligned}
& T=F_{0}=B^{j} F_{j} B^{-j}, \quad j \geq 0, \\
& U=F_{-1}=B^{j+1} F_{j} B^{-(\jmath+1)}, \quad j \geq 0 .
\end{aligned}
$$

Lemma 2.2 says that conjugating by $B^{\jmath}$ lowers the index of the strings by $j$.

Proof. The assertions follow by iterating Lemma 2.1.

Let $\Sigma$ act on symbol strings by means of the substitution $U \mapsto T$ and $T \mapsto U T$. The operator $\Sigma$ can be used to define the Fibonacci strings recursively:

\section{Lemma 2.3.}

$$
F_{\jmath+1}=\Sigma\left(F_{\jmath}\right), \quad j \geq-1 .
$$

Prof. For $j=-1$, we have $F_{0}=T=\Sigma(U)=\Sigma\left(F_{-1}\right)$. For $j=0$, we have $F_{1}=U T=\Sigma(T)=\Sigma\left(F_{0}\right)$ 
Proceeding by induction, we obtain

$$
F_{j+2}=F_{\jmath} F_{j+1}=\Sigma\left(F_{j-1}\right) \Sigma\left(F_{\jmath}\right)=\Sigma\left(F_{j-1} F_{j}\right)=\Sigma\left(F_{j+1}\right)
$$

for $j \geq 0$.

Lemma 2.4. For any string of the symbols $U$ and $T$, we have

$$
\Sigma(F)=B^{-1} F B \text {. }
$$

Lemma 2.4 says that $\Sigma$ acts formally by conjugation by $B^{-1}$.

Proof. The claim follows immediately form the identities

$$
B^{-1} U B=T=\Sigma(U)
$$

and

$$
B^{-1} T B=U T=\Sigma(T),
$$

which follow from the fixed point equation.

We now introduce what we call Fibonacci tails; these are tails of Fibonacci strings. We shall write $\left\{F_{j}\right\}$ for the set of tails of the $j^{\text {th }}$ Fibonacci string; this set is meant to include the empty string but not the $j^{\text {th }}$ Fibonacci string itself. Since the Fibonacci strings grow to the left, there is exactly one Fibonacci tail of each length.

Now, we are ready for the explicit expression for the sets $L_{j}$ :

\section{Lemma 2.5 .}

$$
L_{\jmath}=\left\{F_{j}\right\} B^{-\jmath} D_{T} \cup\left\{F_{j-1}\right\} B^{-j} D_{U}, \quad j \geq 0 .
$$

Lemma 2.5 say that $L_{j}$ is a union of two sets, each of which is a Fibonacci orbit, excluding the last iteration, of a small open set.

Proof. Denoting the empty string by $\diamond$, we have $\left\{F_{-1}\right\}=\left\{F_{0}\right\}=\{\diamond\}$ and $\left\{F_{1}\right\}=$ $\{\diamond, T\}$. Moreover, $L_{0}=D_{U} \cup D_{T}$ and $L_{1}=M_{1} \cup N_{1}=T B^{-1} D_{T} \cup B^{-1}\left(D_{U} \cup D_{T}\right)$.

For $j=0$, the assertion therefore is $M_{0} \cup N_{0}=D_{T} \cup D_{U}$, which is true. For $j=1$, the assertion is $M_{1} \cup N_{1}=B^{-1} D_{T} \cup T B^{-1} D_{T} \cup B^{-1} D_{U}$, which is true as well.

Using Lemma 1.5, we obtain inductively

$$
\begin{aligned}
L_{j+2}= & B^{-1} L_{j+1} \cup T B^{-2} L_{j} \\
= & B^{-1}\left\{F_{j+1}\right\} B^{-(j+1)} D_{T} \cup B^{-1}\left\{F_{j}\right\} B^{-(j+1)} D_{U} \\
& \cup T B^{-2}\left\{F_{j}\right\} B^{-j} D_{T} \cup T B^{-2}\left\{F_{j-1}\right\} B^{-j} D_{U} \\
= & B^{-1}\left\{F_{\jmath+1}\right\} B B^{-(j+2)} D_{T} \cup T B^{-2}\left\{F_{j}\right\} B^{2} B^{-(j+2)} D_{T} \\
& \cup B^{-1}\left\{F_{j}\right\} B B^{-(j+2)} D_{U} \cup T B^{-2}\left\{F_{j-1}\right\} B^{2} B^{-(j+2)} D_{U} \\
= & {\left[\Sigma\left(\left\{F_{j+1}\right\}\right) \cup T \Sigma^{2}\left(\left\{F_{\jmath}\right\}\right)\right] B^{-(j+2)} D_{T} } \\
& \cup\left[\Sigma\left(\left\{F_{j}\right\}\right) \cup T \Sigma^{2}\left(\left\{F_{\jmath-1}\right\}\right)\right] B^{-(j+2)} D_{U}
\end{aligned}
$$

for $j \geq 0$, (where we have used Lemma 2.4 in the last step), and the proof is reduced to the combinatorial identity

$$
\left\{F_{j+2}\right\}=\Sigma\left(\left\{F_{j+1}\right\}\right) \cup T \Sigma^{2}\left(\left\{F_{j}\right\}\right), \quad j \geq-1,
$$

which will be proved in Lemma 2.8 . 
Let us introduce a little bit more terminology. To each Fibonacci tail $F$, there exists a unique successor $G$. We define the symbol valued function $\nu(F)$ to be equal to $U$ or to $T$, according to whether $G$ starts with $U$ or $T$. The symbol $\nu(F)$ will be called the next symbol of the string $F$. Furthermore, let $\left\{F_{j}\right\}_{U}$ be the level set of the function $\nu$ on $\left\{F_{j}\right\}$ corresponding to the level $U$, and $\left\{F_{j}\right\}_{T}$ be the level set corresponding to the level $T$; this simply means that for $F \in\left\{F_{j}\right\}_{U}$, the next symbol is $U$, and for $F \in\left\{F_{\jmath}\right\}_{T}$, the next symbol is $T$.

Lemma 2.6. Let $F$ be any Fibonacci tail. Then we have the following equations:

$$
\nu(\Sigma(F))=T, \quad \nu\left(T \Sigma^{2}(F)\right)=U .
$$

Proof. Since $F$ is a Fibonacci tail, there exists a Fibonacci string $F_{j}$ and a string $\Delta$, different from the empty string, such that $F_{\jmath}=\Delta F$. This implies $F_{j+1}=\Sigma\left(F_{j}\right)=$ $\Sigma(\Delta) \Sigma(F)$. Because of $\Sigma(U)=T$ and $\Sigma(T)=U T$, it follows that each string (different from the empty string) in the image of $\Sigma$ ends with the symbol $T$. In particular, this holds for the string $\Sigma(\Delta)$, and we have shown that $\nu(\Sigma(F))=T$.

For the proof of the second assertion, we observe that

$$
F_{j+2}=\Sigma^{2}\left(F_{j}\right)=\Sigma^{2}(\Delta) \Sigma^{2}(F) .
$$

Because of $\Sigma^{2}(U)=U T$ and $\Sigma^{2}(T)=T U T$, it follows that each string in the image of $\Sigma^{2}$ ends with the symbols $U T$. In particular, this holds for the string $\Sigma^{2}(\Delta)$, and we have shown that $\nu\left(T \Sigma^{2}(F)\right)=U$.

Lemma 2.7. For $j \geq-1$, the following inclusions hold:

$$
\Sigma\left(\left\{F_{j+1}\right\}\right) \subset\left\{F_{j+2}\right\}, \quad T \Sigma^{2}\left(\left\{F_{j}\right\}\right) \subset\left\{F_{j+2}\right\} .
$$

Proof. In order to prove the first inclusion, we take $F \in\left\{F_{j+1}\right\}$ and let $\Delta$ be the difference string to the Fibonacci string $F_{j+1}$. Then we have $F_{\jmath+1}=\Delta F$, and it follows that $F_{\jmath+2}=\Sigma\left(F_{\jmath+1}\right)=\Sigma(\Delta) \Sigma(F)$; therefore, $\Sigma(F)$ is a tail of the Fibonacci string $F_{j+2}$.

For the proof of the second inclusion, we take $F \in\left\{F_{\jmath}\right\}$ and let $\Delta$ be the difference string to the Fibonacci string $F_{j}$. Then we have $F_{j}=\Delta F$, and it follows that $F_{j+2}=\Sigma^{2}\left(F_{j}\right)=\Sigma^{2}(\Delta) \Sigma^{2}(F)$. Therefore, $\Sigma^{2}(F)$ is a tail of the Fibonacci string $F_{j+2}$. Furthermore, by the definition of $\Sigma$, the length of the string $\Sigma^{2}(\Delta)$ is at least 2; it follows that either $U \Sigma^{2}(F)$ or $T \Sigma^{2}(F)$ is a tail of $F_{\jmath+2}$. Lemma 2.6 rules out the first case, and we have shown that $T \Sigma^{2}(F) \in\left\{F_{\jmath+2}\right\}$.

Lemma 2.8. For $j \geq-1$, the following identity holds:

$$
\left\{F_{j+2}\right\}=\Sigma\left(\left\{F_{\jmath+1}\right\}\right) \cup T \Sigma^{2}\left(\left\{F_{\jmath}\right\}\right) .
$$

Proof. By the preceding lemma, we know that the right-hand side is contained in the left-hand side. Since $\operatorname{card}\left\{F_{j+2}\right\}=\operatorname{card}\left\{F_{j+1}\right\}+\operatorname{card}\left\{F_{j}\right\}$ (observe that $\operatorname{card}\left\{F_{j}\right\}=$ length $\left.\left(F_{\jmath}\right)\right)$, it is sufficient to show that $\Sigma\left(\left\{F_{\jmath+1}\right\}\right) \cap T \Sigma^{2}\left(\left\{F_{\jmath}\right\}\right)=\emptyset$. Assume to the contrary that $F \in\left\{F_{j+1}\right\}$ and $G \in\left\{F_{j}\right\}$ and that

$$
\Sigma(F)=T \Sigma^{2}(G) .
$$

Lemma 2.6 implies that the next symbol of the left-hand side is $T$ and the next symbol of the right-hand side is $U$, which is a contradiction. 
Finally, we formulate a sharper version of Lemma 2.5.

Lemma 2.9. We have

$$
\begin{aligned}
& M_{\jmath}=\left\{F_{\jmath}\right\}_{U} B^{-\jmath} D_{T} \cup\left\{F_{j-1}\right\}_{U} B^{-j} D_{U}, \\
& N_{j}=\left\{F_{j}\right\}_{T} B^{-\jmath} D_{T} \cup\left\{F_{\jmath-1}\right\}_{T} B^{-j} D_{U},
\end{aligned}
$$

for $j \geq 1$.

Lemma 2.9. says that $M_{j}$ consists of the patches for which the next symbol is $U$, and $N$, of those for which it is $T$.

Proof. For $j=1$, we have $M_{1}=T B^{-1} D_{U}$ and $N_{1}=B^{-1} D_{T} \cup B^{-1} D_{U}$. Since the next symbol of the string $T$ is $U$ and the next symbol of the empty string is $T$, the claim is true.

Fix $j \geq 1$ and take any $z \in M_{j+1}$. We have

$$
z \in M_{j+1}=T B^{-1} N_{j}=T B^{-2}\left(M_{j-1} \cup N_{j-1}\right)=T B^{-2} L_{\jmath-1}
$$

by definition. It follows from Lemma 2.5 that

$$
z \in T B^{-2} F B^{-(j-1)} D=T B^{-2} F B^{2} B^{-(\jmath+1)} D,
$$

where $F$ is a Fibonacci tail, and where $D$ is one of the two sets $D_{U}$ and $D_{T}$. By Lemma 2.4, we have $T B^{-2} F B^{2}=T \Sigma^{2}(F)$. Lemma 2.6 tells us that the next symbol of $T \Sigma^{2}(F)$ is $U$.

Now, take any $z \in N_{\jmath+1}$. We have

$$
z \in N_{\jmath+1}=B^{-1}\left(M_{j} \cup N_{j}\right)=B^{-1} L_{\jmath}
$$

by definition. It follows from Lemma 2.5 that

$$
z \in B^{-1} F B^{-\jmath} D=B^{-1} F B B^{-(j+1)} D,
$$

where again $F$ is a Fibonacci tail and where $D$ is one of the two sets $D_{U}$ and $D_{T}$. By Lemma 2.4, we have $B^{-1} F B=\Sigma(F)$. Lemma 2.6 tells us that the next symbol of $\Sigma(F)$ is $T$.

\section{Approximate Invariant Sets}

It follows from the relation $B^{-1} \overline{D_{T}} \subset D_{T}$ that the sets $B^{-j} D_{T}$ with $j \geq 1$ are subsets of $B^{-1} \overline{D_{T}}$. Since, by assumption $\mathrm{A} 1$, the diagonal elements of $B$ are of absolute value greater than one, these sets converge to the point $(0,0)$, and it follows that this point is contained in $B^{-1} \overline{D_{T}} \subset D_{T}$. Let $\{F\}$ be the set of all Fibonacci tails. The point set $\{F\}(0,0)$ will be called the orbit of the point $(0,0)$ under the pair $(U, T)$. Since Lemma 2.2 gives $B^{j} F_{j} B^{-j}=F_{0}=T$, the image $F_{j}(0,0)=B^{-j} T(0,0)$ is well defined for all $j$, and we see that the full orbit of $(0,0)$ is well defined.

We should like to regard the orbit of $(0,0)$ as an invariant set for the pair $(U, T)$. But it is a priori not clear what invariant set means in this context. It turns out that it is more natural work with invariant pairs of sets.

Definition 3.1. A pair $(M, N)$ of sets is called invariant for a pair $(U, T)$ of maps if

$$
U M \subset M \cup N \text { and } T N \subset M \cup N \text {. }
$$


Of course, the pairs $\left(M_{\jmath}, N_{\jmath}\right)$ defined in the first section are not invariant for $(U, T)$. But they are approximately invariant in a sense given by the following definition:

Definition 3.2. A decreasing sequence $\left(\left(M_{\jmath}, N_{\jmath}\right)\right)$ of pairs of sets is called aproximately invariant for a pair $(U, T)$ of maps if for each $j$, there is a $k \geq j$ such that

$$
U M_{k} \subset M_{\jmath} \cup N_{\jmath} \text { and } T N_{k} \subset M_{j} \cup N_{j} .
$$

(By the term "decreasing sequence $\left(\left(M_{\jmath}, N_{\jmath}\right)\right)$ of pairs of sets," we mean that the sequences $\left(M_{j}\right)$ and $\left(N_{j}\right)$ decrease separately.)

Lemma 3.3. Under the assumptions $\mathrm{A} 1, \mathrm{~A} 2$, and $\mathrm{A} 3$, the sequence of the domain pairs is approximately invariant for the pair $(U, T)$.

Proof. Fix $j$ and choose $k \geq j$ such that

$$
B^{-k}\left(T D_{T} \cup U D_{U}\right) \subset B^{-j} D_{T} .
$$

Since $B^{-j} D_{T}$ is an open neighborhood of the origin, and since the set $T D_{T} \cup U D_{U}$ is bounded, this is possible.

Take any point $z \in M_{k}$. We are going to show that $U z \subset L_{\jmath}$.

By Lemma 2.5, we have either $z \in F B^{-k} D_{T}$ or $z \in G B^{-k} D_{U}$, where $F$ and $G$ are tails of the Fibonacci strings $F_{k}$ or $F_{k-1}$ respectively. Assume for instance that $z \in F B^{-k} D_{T}$. (For $z \in G B^{-k} D_{U}$, the reasoning is completely analogous.)

Since $z \in M_{k}$ by assumption, the next symbol of $F$ is $U$ by Lemma 2.9. If $F$ is not maximal, i.e., if its successor is not the Fibonacci string $F_{k}$, then the string $U F$ is a tail of the Fibonacci string $F_{k}$, and therefore $U z$ is contained in $L_{k}$ and a fortiori in $L_{j}$.

If, however, $F$ is maximal, then we have $U F=F_{k}$, and if follows from Lemma 2.2 that $U z \in F_{k} B^{-k} D_{T}=B^{-k} B^{k} F_{k} B^{-k} D_{T}=B^{-k} F_{0} D_{T}=B^{-k} T D_{T}$. In the case where $z \in G B^{-k} D_{U}$, it follows in the same way that $U z$ is contained in $B^{-k} U D_{U}$.

By Lemma 2.5, the set $B^{-j} D_{T}$ belongs to $L_{j}$. By the definition of $k$, we therefore have $U z \in L_{j}$.

We have shown: $U M_{k} \subset L_{\jmath}$. The proof of $T N_{k} \subset L_{\jmath}$ is completely analogous.

The justification of Definition 3.2 lies in the following result:

Lemma 3.4. The limit pair of an approximately invariant sequence is invariant.

Proof. Let $\left(\left(M_{j}, N_{j}\right)\right)$ be the approximately invariant sequence and let

$$
(M, N)=\left(\bigcap_{j} M_{\jmath}, \bigcap_{j} N_{j}\right)
$$

be its limit pair. Fix $z \in M$ and take an arbitrary $j$. We show $U z \in M_{j} \cup N_{j}$. There exists a $k \geq j$ such that if $w \in M_{k}$ then $U w \in M_{\jmath} \cup N_{\jmath}$. Since $z \in M$, it lies in $M_{k}$ as well, and we have $U z \in M_{\jmath} \cup N_{\jmath}$.

Since $j$ and $z$ were arbitrary, we have proved that $U M \subset M \cup N$. The proof of $T N \subset M \cup N$ is entirely analogous.

Theorem 3.5. Under the assumptions A1, A2, and A3, the self-similar pair $(U, T)$ has a connected compact invariant pair containing the orbit of the point $(0,0)$.

Proof. Because of Lemma 3.3 and Lemma 3.4, the limit pair $(M, N)$ is an invariant pair for $(U, T)$. 
In order to prove that the orbit of $(0,0)$ is contained in $L$, we fix $j$ and show that $\left\{F_{\jmath}\right\}(0,0) \subset L$. Since $(0,0) \in B^{-k} D_{T}$, we have $\left\{F_{\jmath}\right\}(0,0) \subset L_{k}$ for all $k \geq j$ by Lemma 2.5. We conclude that $\left\{F_{\jmath}\right\}(0,0) \subset L$, and since $j$ was arbitrary, the claim follows.

\section{Asymptotic Self-Similarity}

The aim of this section is to show that the conclusions of Theorem 3.5 hold under the weaker assumption of asymptotic self-similarity. The argumentation is exactly the same as in the first three sections, and the proofs stay almost verbatim the same. We include them nevertheless.

A pair $(U, T)$ is called self-similar if it is a fixed point of the renormalization operator. A pair $\left(U_{0}, T_{0}\right)$ is called asymptotically self-similar if its orbit under the renormalization operator converges to a fixed point. This orbit looks as follows:

$$
\left(U_{k+1}, T_{k+1}\right)=B_{k+1}\left(T_{k}, U_{k} T_{k}\right) B_{k+1}^{-1}, \quad k \geq 0 .
$$

The index $k$ denotes the iteration step number along the orbit; it will keep this meaning in the whole section.

The maps $B_{k+1}$ are assumed to be linear-diagonal and the maps $U_{k}$ and $T_{k}$ to be homeomorphisms on the domains $D_{U}$ and $D_{T}$ respectively. By convergence to a fixed point we mean the following: the sequences $\left(U_{k}\right)$ and $\left(T_{k}\right)$ converge uniformly on their domains, and the diagonal elements of the maps $B_{k+1}$ converge.

From these assumptions, it follows that the limit maps, denoted by $U, T$, and $B$, satisfy the fixed point equation from Sect. 1. Again, we shall assume that the diagonal elements of the map $B$ are of absolute value greater than one (A1) and that the renormalization operator extends the domains (A2):

$$
B^{-1} \overline{D_{U}} \subset D_{T}, \quad B^{-1} \overline{D_{T}} \subset D_{T}, \quad T B^{-1} \overline{D_{T}} \subset D_{U}
$$

From this and from the convergence to the fixed point, it follows that analogous assumptions hold for pairs $\left(U_{k}, T_{k}\right)$ with $k$ larger than a certain $k_{0}$; without loss of generality, we take $k_{0}$ equal to zero. Specifically, we shall make use of the following conditions:

$\mathrm{A} 1^{\prime}$. The diagonal elements of the maps $B_{k+1}$ are of abolute value greater than and bounded away from one for all non-negative integers $k$.

$\mathrm{A} 2^{\prime}$.

$$
B_{k+1}^{-1} \overline{D_{U}} \subset D_{T}, \quad B_{k+1}^{-1} \overline{D_{T}} \subset D_{T}, \quad T_{k} B_{k+1}^{-1} \overline{D_{T}} \subset D_{U}
$$

for all non-negative integers $k$.

In particular, it follows from this that the sets $T_{k+1} D_{T}$ and $U_{k+1} D_{U}$ are bounded uniformly for $k \geq 0$. Moreover, it follows that the equation $U_{k+1}=B_{k+1} T_{k} B_{k+1}^{-1}$ extends $U_{k+1}$ to a homeomorphism on $D_{U} \cup D_{T}$. From now on we take $U_{k+1}$ to be this extension, i.e., we assume that $U_{k+1}$ is defined on $D_{T}$ as well.

We now consider a doubly infinite sequence of domains pairs $\left(M_{j}^{k}, N_{\jmath}^{k}\right)$, defined by $M_{0}^{k}=D_{U}, N_{0}^{k}=D_{T}$ for all non-negative integers $k$ and

$$
M_{j+1}^{k}=T_{k} B_{k+1}^{-1} N_{\jmath}^{k+1}, \quad N_{j+1}^{k}=B_{k+1}^{-1}\left(M_{\jmath}^{k+1} \cup N_{j}^{k+1}\right)
$$

for $j \geq 0$, so long as $T_{k} B_{k+1}^{-1}$ is well defined on $N_{\jmath}^{k+1}$ for all non-negative integers $k$. 
Lemma 4.1. Under the assumption $\mathrm{A} 2^{\prime}$, the pairs $\left(M_{j}^{k}, N_{j}^{k}\right)$ are well defined for all non-negative integers $j$ and $k$, and the following relations hold:

$$
\overline{M_{j+1}^{k}} \subset M_{\jmath}^{k}, \quad \overline{N_{j+1}^{k}} \subset N_{\jmath}^{k}
$$

for all pairs $(j, k)$ of non-negative integers.

Proof. We prove the claims

$$
T_{k} B_{k+1}^{-1} \overline{N_{\jmath}^{k+1}} \subset M_{\jmath}^{k}, \quad B_{k+1}^{-1} \overline{M_{\jmath}^{k+1}} \subset N_{j}^{k}, \quad B_{k+1}^{-1} \overline{N_{j}^{k+1}} \subset N_{j}^{k}
$$

by induction on $j$. For $j=0$, they are identical to the domain extension property. We have $B_{k+1}^{-1} \overline{N_{0}^{k+1}}=B_{k+1}^{-1} \overline{D_{T}} \subset D_{T}$; therefore, $M_{1}^{k}$ is well defined for all $k .\left(N_{1}^{k}\right.$ is well defined anyway.)

Let $j$ be greater than or equal to zero; we assume that the claims have been proved up to $j$ and that $M_{j+1}^{k}$ and $N_{j+1}^{k}$ are well defined. Then we have

$$
\begin{aligned}
T_{k} B_{k+1}^{-1} \overline{N_{\jmath+1}^{k+1}}= & T_{k} B_{k+1}^{-1} B_{k+2}^{-1} \overline{M_{j}^{k+2}} \cup T_{k} B_{k+1}^{-1} B_{k+2}^{-1} \overline{N_{\jmath}^{k+2}} \\
& \subset T_{k} B_{k+1}^{-1} N_{j}^{k+1}=M_{j+1}^{k}
\end{aligned}
$$

by the definitions of $N_{j+1}^{k}$ and of $M_{\jmath+1}^{k}$ and by the inductive assumption. In the same way, we obtain

and

$$
B_{k+1}^{-1} \overline{M_{j+1}^{k+1}}=B_{k+1}^{-1} T_{k+1} B_{k+2}^{-1} \overline{N_{j}^{k+2}} \subset B_{k+1}^{-1} M_{j}^{k+1} \subset N_{j+1}^{k}
$$

$$
B_{k+1}^{-1} \overline{N_{j+1}^{k+1}}=B_{k+1}^{-1} B_{k+2}^{-1} \overline{M_{j}^{k+2}} \cup B_{k+1}^{-1} B_{k+2}^{-1} \overline{N_{j}^{k+2}} \subset B_{k+1}^{-1} N_{j}^{k+1} \subset N_{j+1}^{k} .
$$

In particular, it follows that

$$
B_{k+1}^{-1} \overline{N_{\jmath+1}^{k+1}} \subset B_{k+1}^{-1} N_{\jmath}^{k+1} \subset B_{k+1}^{-1} N_{0}^{k+1}=B_{k+1}^{-1} D_{T} \subset D_{T},
$$

which shows that $M_{j+2}^{k}$ is well defined for all $k$.

[For this proof, the definition of the pairs $\left(U_{k}, T_{k}\right)$ is not needed.]

We have shown that for each fixed $k$, the sets $\overline{M_{j}^{k}}$ and $\overline{N_{j}^{k}}$ form decreasing sequences of non-empty compact sets. As in Sect. 1, we now impose a connectedness condition on the fixed point $(U, T)$, namely (A3),

$$
D_{U} \cap D_{T} \neq \emptyset, \quad U D_{T} \cap D_{T} \neq \emptyset, \quad U B^{-1} D_{T} \cap B^{-1} D_{U} \neq \emptyset .
$$

Again, from convergence to the fixed point, it follows that analogous relations hold for pairs $\left(U_{k}, T_{k}\right)$ with $k$ larger than a certain fixed $k_{0}$, which is again taken to be zero. Specifically, we shall make use of the following conditions:

$\mathrm{A} 3^{\prime}$.

$$
D_{U} \cap D_{T} \neq \emptyset, \quad U_{k} D_{T} \cap D_{T} \neq \emptyset, \quad U_{k} B_{k+1}^{-1} D_{T} \cap B_{k+1}^{-1} D_{U} \neq \emptyset
$$

for all non-negative integers $k$. (Remember that $U_{k}$ is defined on $D_{T}$ as well for $k \geq 1$; without loss of generality, we assume that this is true for $k=0$ too.) These assumptions will be called connectedness assumptions.

Lemma 4.2. Under the assumptions $\mathrm{A} 2^{\prime}$ and $\mathrm{A} 3^{\prime}$, the following relations hold:

$$
U_{k} N_{j}^{k} \cap N_{j}^{k} \neq \emptyset, \quad U_{k} B_{k+1}^{-1} N_{j}^{k+1} \cap B_{k+1}^{-1} M_{\jmath}^{k+1} \neq \emptyset
$$

for all pairs $(j, k)$ of non-negative integers. 
Proof. For $j=0$, these claims correspond to the second and third connectedness assumption. Proceeding by induction on $j$, we obtain

$$
\begin{aligned}
U_{k} N_{j+1}^{k} \cap N_{\jmath+1}^{k} & =U_{k}\left(B_{k+1}^{-1} M_{j}^{k+1} \cup B_{k+1}^{-1} N_{j}^{k+1}\right) \cap\left(B_{k+1}^{-1} M_{j}^{k+1} \cup B_{k+1}^{-1} N_{j}^{k+1}\right) \\
& \supset U_{k} B_{k+1}^{-1} N_{\jmath}^{k+1} \cap B_{k+1}^{-1} M_{j}^{k+1} \neq \emptyset
\end{aligned}
$$

for arbitrary $k$. Making use of the defining equation $T_{k+1}=B_{k+1} U_{k} T_{k} B_{k+1}^{-1}$, we obtain furthermore

$$
\begin{aligned}
U_{k} & B_{k+1}^{-1} N_{j+1}^{k+1} \cap B_{k+1}^{-1} M_{j+1}^{k+1} \\
& =U_{k} B_{k+1}^{-1}\left(B_{k+2}^{-1} M_{j}^{k+2} \cup B_{k+2}^{-1} N_{j}^{k+2}\right) \cap B_{k+1}^{-1} T_{k+1} B_{k+2}^{-1} N_{j}^{k+2} \\
& =U_{k} B_{k+1}^{-1} B_{k+2}^{-1}\left(M_{j}^{k+2} \cup N_{\jmath}^{k+2}\right) \cap B_{k+1}^{-1} B_{k+1} U_{k} T_{k} B_{k+1}^{-1} B_{k+2}^{-1} N_{j}^{k+2} \\
& \supset U_{k}\left(B_{k+1}^{-1} B_{k+2}^{-1} M_{\jmath}^{k+2} \cap T_{k} B_{k+1}^{-1} B_{k+2}^{-1} N_{j}^{k+2}\right) \\
& =U_{k}\left(B_{k+1}^{-1} B_{k+2}^{-1} M_{j}^{k+2} \cap B_{k+1}^{-1} B_{k+1} T_{k} B_{k+1}^{-1} B_{k+2}^{-1} N_{j}^{k+2}\right) \\
& =U_{k} B_{k+1}^{-1}\left(B_{k+2}^{-1} M_{j}^{k+2} \cap U_{k+1} B_{k+2}^{-1} N_{\jmath}^{k+2}\right) .
\end{aligned}
$$

The last set on the right-hand side is not empty by the inductive assumption.

Lemma 4.3. Under the assumptions $\mathrm{A} 2^{\prime}$ and $\mathrm{A} 3^{\prime}$, we have

$$
M_{j}^{k} \cap N_{j}^{k} \neq \emptyset
$$

for all pairs $(j, k)$ of non-negative integers.

Proof. For $j=0$, the claim amounts to the relation $D_{U} \cap D_{T} \neq \emptyset$, which is true by the first connectedness assumption.

We have $M_{j+1}^{k}=T_{k} B_{k+1}^{-1} N_{j}^{k+1}=B_{k+1}^{-1} U_{k+1} N_{j}^{k+1}$ by the definition of $U_{k+1}$. (Remember that we assume $U_{k+1}$ to be defined on $D_{T}$ too, and that $N_{j}^{k+1} \subset D_{T}$ by Lemma 4.1.) Since $N_{j+1}^{k}=B_{k+1}^{-1} M_{j}^{k+1} \cup B_{k+1}^{-1} N_{j}^{k+1}$, we obtain

$$
N_{j+1}^{k} \cap M_{j+1}^{k} \supset B_{k+1}^{-1} N_{j}^{k+1} \cap B_{k+1}^{-1} U_{k+1} N_{j}^{k+1}=B_{k+1}^{-1}\left(N_{j}^{k+1} \cap U_{k+1} N_{j}^{k+1}\right) \neq \emptyset
$$

by Lemma 4.2 .

Lemma 4.4. Under the assumptions $\mathrm{A} 2^{\prime}$ and $\mathrm{A} 3^{\prime}$, the sets $M_{j}^{k}$ and $N_{k}^{k}$ are connected for all pairs $(j, k)$ of non-negative integers.

Proof. The proof is exactly the same as the one of Lemma 1.4.

Now, we define the sets $L_{j}^{k}$ by $L_{j}^{k}=M_{j}^{k} \cup N_{j}^{k}$.

Lemma 4.5. The sets $L_{j}^{k}$ satisfy the following recursion relation:

$$
L_{j+2}^{k}=B_{k+1}^{-1} L_{j+1}^{k+1} \cup T_{k} B_{k+1}^{-1} B_{k+2}^{-1} L_{j}^{k+2}
$$

for all pairs $(j, k)$ of non-negative integers.

Proof. By definition, we have

$$
\begin{aligned}
L_{j+2}^{k} & =M_{j+2}^{k} \cup N_{j+2}^{k} \\
& =T_{k} B_{k+1}^{-1} N_{j+1}^{k+1} \cup B_{k+1}^{-1}\left(M_{j+1}^{k+1} \cup N_{j+1}^{k+1}\right) \\
& =T_{k} B_{k+1}^{-1} B_{k+2}^{-1}\left(N_{j}^{k+2} \cup M_{j}^{k+2}\right) \cup B_{k+1}^{-1} L_{j+1}^{k+1} \\
& =T_{k} B_{k+1}^{-1} B_{k+2}^{-1} L_{j}^{k+2} \cup B_{k+1}^{-1} L_{j+1}^{k+1}
\end{aligned}
$$

for all pairs $(j, k)$ of non-negative integers. 
Finally, we take the limit pair of the $\left(M_{j}^{k}, N_{j}^{k}\right)$ :

$$
\left(M^{k}, N^{k}\right)=\left(\bigcap_{j} M_{j}^{k}, \bigcap_{j} N_{j}^{k}\right)
$$

The union of $M^{k}$ and $N^{k}$ will be denoted by $L^{k}$. We have

$$
L^{k}=M^{k} \cup N^{k}=\bigcap_{j}\left(M_{\jmath}^{k} \cup N_{j}^{k}\right)=\bigcap_{\jmath} L_{j}^{k} .
$$

(Remember that the sequences $\left(M_{j}^{k}\right)$ and $\left(N_{j}^{k}\right)$ are decreasing.)

Lemma 4.6. Under the assumptions $\mathrm{A} 2^{\prime}$ and $\mathrm{A} 3^{\prime}$, the limit pairs $\left(M^{k}, N^{k}\right)$ thus defined are non-empty, compact, and connected.

Proof. The proof is exactly the same as the one of Lemma 1.6.

The Fibonacci strings from Sect. 2 now acquire a second index $k$; formally, they are defined as follows:

$$
\begin{gathered}
F_{-1}^{k}=U_{k}, \quad F_{0}^{k}=T_{k}, \\
F_{j+2}^{k}=F_{j}^{k} F_{j+1}^{k}, \quad j \geq-1
\end{gathered}
$$

for all non-negative integers $k$.

Let us introduce the following shorthand notations:

$$
\begin{gathered}
B_{k+1}^{-j}=B_{k+1}^{-1} B_{k+2}^{-1} \ldots B_{k+j}^{-1}, \\
B_{k+1}^{j}=B_{k+j} B_{k+j-1} \ldots B_{k+1} .
\end{gathered}
$$

Lemma 4.7. The recursive definition of the pairs $\left(U_{k+1}, T_{k+1}\right)$ translates into

$$
F_{j}^{k+1}=B_{k+1} F_{j+1}^{k} B_{k+1}^{-1}, \quad j \geq-1
$$

for all non-negative integers $k$.

Proof. For $j=-1$ and $j=0$, the assertions correspond to the definition of the pairs $\left(U_{k+1}, T_{k+1}\right)$.

Proceeding by induction on $j$, we obtain

$$
\begin{aligned}
F_{j+1}^{k+1} & =F_{\jmath-1}^{k+1} F_{\jmath}^{k+1}=B_{k+1} F_{\jmath}^{k} B_{k+1}^{-1} B_{k+1} F_{\jmath+1}^{k} B_{k+1}^{-1} \\
& =B_{k+1} F_{j}^{k} F_{j+1}^{k} B_{k+1}^{-1}=B_{k+1} F_{\jmath+2}^{k} B_{k+1}^{-1}
\end{aligned}
$$

for $j \geq 0$.

\section{Lemma 4.8.}

$$
\begin{gathered}
T_{k+j}=F_{0}^{k+j}=B_{k+1}^{j} F_{j}^{k} B_{k+1}^{-\jmath}, \quad j \geq 0, \\
U_{k+j+1}=F_{-1}^{k+j+1}=B_{k+1}^{\jmath+1} F_{\jmath}^{k} B_{k+1}^{-(j+1)}, \quad j \geq 0 .
\end{gathered}
$$

Lemma 4.8 says that conjugating by $B_{k+1}^{j}$ lowers the first index by $j$, but only at the expense of raising the second index by the same amount.

Proof. The assertions follow by iterating Lemma 4.7 . 
Let $\Sigma$ act on symbol strings by means of the substitution $U_{k} \mapsto T_{k}$ and $T_{k} \mapsto U_{k} T_{k}$. Since $\Sigma$ does not affect the index $k$, Lemma 2.3 stays valid in the following form:

$$
F_{j+1}^{k}=\Sigma\left(F_{j}^{k}\right), \quad j \geq-1
$$

for all non-negative integers $k$.

Lemma 4.9. Let $F^{k}$ be any string of the symbols $U_{k}$ and $T_{k}$, and let $F^{k+1}$ be the same string of the symbols $U_{k+1}$ and $T_{k+1}$. Then we have

$$
\Sigma\left(F^{k}\right)=B_{k+1}^{-1} F^{k+1} B_{k+1}
$$

Lemma 4.9 says that $\Sigma$ acts formally by conjugation with $B_{k+1}^{-1}$ and by an index shift.

Proof. The claim immediately follows from the identities

$$
B_{k+1}^{-1} U_{k+1} B_{k+1}=T_{k}=\Sigma\left(U_{k}\right)
$$

and

$$
B_{k+1}^{-1} T_{k+1} B_{k+1}=U_{k} T_{k}=\Sigma\left(T_{k}\right)
$$

which follow from the definition of the pairs $\left(U_{k+1}, T_{k+1}\right)$.

Again, we introduce the Fibonacci tails as tails of Fibonacci strings. We shall write $\left\{F_{j}^{k}\right\}$ for the set of tails the $j^{\text {th }}$ Fibonacci string of the symbols $U_{k}$ and $T_{k}$; this set is meant to include the empty string but not the $j^{\text {th }}$ Fibonacci string itself.

Remember the shorthand notations introduced above:

$$
\begin{gathered}
B_{k+1}^{-\jmath}=B_{k+1}^{-1} B_{k+2}^{-1} \ldots B_{k+j}^{-1}, \\
B_{k+1}^{j}=B_{k+\jmath} B_{k+j-1} \ldots B_{k+1} .
\end{gathered}
$$

Now we are ready for the explicit expression for the sets $L_{j}^{k}$ :

\section{Lemma 4.10.}

$$
L_{j}^{k}=\left\{F_{j}^{k}\right\} B_{k+1}^{-j} D_{T} \cup\left\{F_{\jmath-1}^{k}\right\} B_{k+1}^{-j} D_{U}, \quad j \geq 0
$$

for all non-negative integers $k$.

Proof. Denoting the empty string by $\diamond$, we have $\left\{F_{-1}^{k}\right\}=\left\{F_{0}^{k}\right\}=\{\diamond\}$ and $\left\{F_{1}^{k}\right\}=\left\{\diamond, T_{k}\right\}$. Moreover, $L_{0}^{k}=D_{U} \cup D_{T}$ and $L_{1}^{k}=M_{1}^{k} \cup N_{1}^{k}=T_{k} B_{k+1}^{-1} D_{T} \cup$ $B_{k+1}^{-1}\left(D_{U} \cup D_{T}\right)$.

For $j=0$, the assertion therefore is $M_{0}^{k} \cup N_{0}^{k}=D_{T} \cup D_{U}$, which is true. For $j=1$, the assertion is $M_{1}^{k} \cup N_{1}^{k}=B_{k+1}^{-1} D_{T} \cup T_{k} B_{k+1}^{-1} D_{T} \cup B_{k+1}^{-1} D_{U}$, which is true as well. 
Using Lemma 4.5, we obtain inductively

$$
\begin{aligned}
L_{j+2}^{k}= & B_{k+1}^{-1} L_{j+1}^{k+1} \cup T_{k} B_{k+1}^{-1} B_{k+2}^{-1} L_{\jmath}^{k+2} \\
= & B_{k+1}^{-1}\left\{F_{j+1}^{k+1}\right\} B_{k+2}^{-(j+1)} D_{T} \cup B_{k+1}^{-1}\left\{F_{j}^{k+1}\right\} B_{k+2}^{-(\jmath+1)} D_{U} \\
& \cup T_{k} B_{k+1}^{-1} B_{k+2}^{-1}\left\{F_{j}^{k+2}\right\} B_{k+3}^{-j} D_{T} \cup T_{k} B_{k+1}^{-1} B_{k+2}^{-1}\left\{F_{j-1}^{k+2}\right\} B_{k+3}^{-j} D_{U} \\
= & B_{k+1}^{-1}\left\{F_{j+1}^{k+1}\right\} B_{k+1} B_{k+1}^{-(j+2)} D_{T} \cup T_{k} B_{k+1}^{-1} B_{k+2}^{-1}\left\{F_{j}^{k+2}\right\} B_{k+2} B_{k+1} B_{k+1}^{-(\jmath+2)} D_{T} \\
& \cup B_{k+1}^{-1}\left\{F_{\jmath}^{k+1}\right\} B_{k+1} B_{k+1}^{-(\jmath+2)} D_{U} \\
& \cup T_{k} B_{k+1}^{-1} B_{k+2}^{-1}\left\{F_{\jmath-1}^{k+2}\right\} B_{k+2} B_{k+1} B_{k+1}^{-(j+2)} D_{U} \\
= & {\left[\Sigma\left(\left\{F_{j+1}^{k}\right\}\right) \cup T_{k} \Sigma^{2}\left(\left\{F_{j}^{k}\right\}\right)\right] B_{k+1}^{-(j+2)} D_{T} } \\
& \cup\left[\Sigma\left(\left\{F_{\jmath}^{k}\right\}\right) \cup T_{k} \Sigma^{2}\left(\left\{F_{j-1}^{k}\right\}\right)\right] B_{k+1}^{-(j+2)} D_{U}
\end{aligned}
$$

for $j \geq 0$, (where we have used Lemma 4.9 in the last step), and the proof is reduced to the combinatorial identity

$$
\left\{F_{j+2}^{k}\right\}=\Sigma\left(\left\{F_{j+1}^{k}\right\}\right) \cup T_{k} \Sigma^{2}\left(\left\{F_{j}^{k}\right\}\right), \quad j \geq-1,
$$

for all non-negative integers $k$, which has been proved in Lemma 2.8 .

Recall from Sect. 2 the definition of the next symbol: the next symbol $\nu(F)$ of a Fibonacci tail $F$ is the first symbol of the Fibonacci tail of minimal length that strictly contains $F$. We denote by $\left\{F_{j}^{k}\right\}_{U}$ (respectively by $\left\{F_{j}^{k}\right\}_{T}$ ) the set of the Fibonacci tails in $\left\{F_{j}^{k}\right\}$ the next symbol of which is $U_{k}$ (respectively $T_{k}$ ).

Lemma 4.11. We have

$$
\begin{gathered}
M_{j}^{k}=\left\{F_{j}^{k}\right\}_{U} B_{k+1}^{-\jmath} D_{T} \cup\left\{F_{j-1}^{k}\right\}_{U} B_{k+1}^{-\jmath} D_{U}, \\
N_{j}^{k}=\left\{F_{j}^{k}\right\}_{T} B_{k+1}^{-j} D_{T} \cup\left\{F_{\jmath-1}^{k}\right\}_{T} B_{k+1}^{-j} D_{U}
\end{gathered}
$$

for $j \geq 1$ and $k \geq 0$.

Proof. Fix $k \geq 0$. For $j=1$, we have $M_{1}^{k}=T_{k} B_{k+1}^{-1} D_{U}$ and $N_{1}^{k}=B_{k+1}^{-1} D_{T} \cup$ $B_{k+1}^{-1} D_{U}$. Since the next symbol of the string $T_{k}$ and $U_{k}$ and the next symbol of the empty string is $T_{k}$, the claim is true.

Fix $j \geq 1$ and take any $z \in M_{j+1}$. We have

$$
\begin{aligned}
z \in M_{j+1}^{k} & =T_{k} B_{k+1}^{-1} N_{j}^{k+1} \\
& =T_{k} B_{k+1}^{-1} B_{k+2}^{-1}\left(M_{j-1}^{k+2} \cup N_{j-1}^{k+2}\right)=T_{k} B_{k+1}^{-1} B_{k+2}^{-1} L_{j-1}^{k+2}
\end{aligned}
$$

by definition. It follows from Lemma 4.10 that

$$
z \in T_{k} B_{k+1}^{-1} B_{k+2}^{-1} F^{k+2} B_{k+3}^{-(j-1)} D=T_{k} B_{k+1}^{-1} B_{k+2}^{-1} F^{k+2} B_{k+2} B_{k+1} B_{k+1}^{-(\jmath+1)} D,
$$

where $F^{k+2}$ is a Fibonacci tail in the symbols $U_{k+2}$ and $T_{k+2}$, and where $D$ is one of the two sets $D_{U}$ and $D_{T}$. We have

$$
T_{k} B_{k+1}^{-1} B_{k+2}^{-1} F^{k+2} B_{k+2} B_{k+1}=T_{k} \Sigma^{2}\left(F^{k}\right)
$$

by Lemma 4.9. Lemma 2.6 tells us that the next symbol of $T_{k} \Sigma^{2}\left(F^{k}\right)$ is $U_{k}$.

Now, take any $z \in N_{j+1}^{k}$. We have

$$
z \in N_{j+1}^{k}=B_{k+1}^{-1}\left(M_{j}^{k+1} \cup N_{j}^{k+1}\right)=B_{k+1}^{-1}\left(L_{j}^{k+1}\right)
$$


by definition. It follows from Lemma 4.9 that

$$
z \in B_{k+1}^{-1} F^{k+1} B_{k+2}^{-\jmath} D=B_{k+1}^{-1} F^{k+1} B_{k+1} B_{k+1}^{-(j+1)} D
$$

where $F^{k+1}$ is a Fibonacci tail in the symbols $U_{k+1}$ and $T_{k+1}$, and where $D$ is one of the two sets $D_{U}$ and $D_{T}$. We have

$$
B_{k+1}^{-1} F^{k+1} B_{k+1}=\Sigma\left(F^{k}\right)
$$

by Lemma 4.9. Lemma 2.6 tells us that the next symbol of $\Sigma\left(F^{k}\right)$ is $T_{k}$.

It follows from the relations $B_{k+1}^{-1} \overline{D_{T}} \subset D_{T}$ that the sets

$$
B_{1}^{-j} D_{T}=B_{1}^{-1} B_{2}^{-1} \ldots B_{\jmath}^{-1} D_{T}
$$

with $j \geq 1$ are subsets of $B_{1}^{-1} \overline{D_{T}}$. Since, by assumption $\mathrm{A} 1^{\prime}$, the diagonal elements of the maps $B_{k+1}$ are of absolute value greater than and bounded away from one, these sets converge to the point $(0,0)$, and it follows that this point is contained in $B_{1}^{-1} \overline{D_{T}} \subset D_{T}$. Let $\left\{F^{0}\right\}$ be the set of all Fibonacci tails of the symbols $U_{0}$ and $T_{0}$. The point set $\left\{F^{0}\right\}(0,0)$ will be called the orbit of the point $(0,0)$ under the pair $\left(U_{0}, T_{0}\right)$. Since Lemma 4.8 gives $B_{1}^{j} F_{\jmath}^{0} B_{1}^{-\jmath}=F_{0}^{\jmath}=T_{j}$, the image $F_{j}^{0}(0,0)=B_{1}^{-j} T_{j}(0,0)$ is well defined for all $j$, and we see that the full orbit of $(0,0)$ is well defined.

Lemma 4.12. Under the assumptions $\mathrm{A}^{\prime}, \mathrm{A} 2^{\prime}$, and $\mathrm{A} 3^{\prime}$, the sequence of pairs $\left(\left(M_{j}^{0}, N_{j}^{0}\right)\right)$ is approximately invariant for the pair $\left(U_{0}, T_{0}\right)$.

Proof. Fix $j$ and choose $k \geq j$ such that

$$
B_{1}^{-k}\left(T_{k} D_{T} \cup U_{k} D_{U}\right) \subset B_{1}^{-j} D_{T} .
$$

Since $B_{1}^{-j} D_{T}$ is an open neighborhood of the origin, and since the sets $T_{k} D_{T} \cup U_{k} D_{U}$ are bounded uniformly, this is possible.

Take any point $z \in M_{k}^{0}$. We are going to show that $U_{0} z \subset L_{\jmath}^{0}$.

By Lemma 4.10, we have either $z \in F B_{1}^{-k} D_{T}$ or $z \in G B_{1}^{-k} D_{U}$, where $F$ and $G$ are tails of the Fibonacci strings $F_{k}^{0}$ or $F_{k-1}^{0}$ respectively. Assume for instance that $z \in F B_{1}^{-k} D_{T}$. (For $z \in G B_{1}^{-k} D_{U}$, the reasoning is completely analogous.)

Since $z \in M_{k}^{0}$ by assumption, the next symbol of $F$ is $U_{0}$ by Lemma 4.11. If $F$ is not maximal, i.e., if its successor is not the Fibonacci string $F_{k}^{0}$, then the string $U_{0} F$ is a tail of the Fibonacci string $F_{k}^{0}$, and therefore $U_{0} z$ is contained in $L_{k}^{0}$ and a fortiori in $L_{\jmath}^{0}$.

If, however, $F$ is maximal, then we have $U_{0} F=F_{k}^{0}$, and it follows from Lemma 4.8 that

$$
U_{0} z \in F_{k}^{0} B_{1}^{-k} D_{T}=B_{1}^{-k} B_{1}^{k} F_{k}^{0} B_{1}^{-k} D_{T}=B_{1}^{-k} F_{0}^{k} D_{T}=B_{1}^{-k} T_{k} D_{T} .
$$

In the case where $z \in G B_{1}^{-k} D_{U}$, it follows in the same way that $U_{0} z$ is contained in $B_{1}^{-k} U_{k} D_{U}$.

By Lemma 4.10, the set $B_{1}^{-j} D_{T}$ belongs to $L_{j}^{0}$. By the definition of $k$, we therefore have $U_{0} z \in L_{j}^{0}$.

We have shown: $U_{0} M_{k}^{0} \subset L_{j}^{0}$. The proof of $T_{0} N_{k}^{0} \subset L_{j}^{0}$ is completely analogous. 
For convenience, we copy Lemma 3.4 from Sect. 3:

Lemma 3.4. The limit pair of an approximately invariant sequence is invariant.

Theorem 4.13. Under the assumptions $\mathrm{A} 1^{\prime}, \mathrm{A} 2^{\prime}$, and $\mathrm{A} 3^{\prime}$, the asymptotically selfsimilar pair $\left(U_{0}, T_{0}\right)$ has a connected compact invariant pair containing the orbit of the point $(0,0)$.

Proof. Because of Lemma 4.12 and Lemma 3.4, the limit pair

$$
\left(M^{0}, N^{0}\right)=\left(\bigcap_{\jmath} M_{\jmath}^{0}, \bigcap_{j} N_{\jmath}^{0}\right)
$$

is an invariant pair for $\left(U_{0}, T_{0}\right)$.

In order to prove that the orbit of $(0,0)$ is contained in $L^{0}$, we fix $j$ and show that $\left\{F_{j}^{0}\right\}(0,0) \subset L^{0}$. Since $(0,0) \in B_{1}^{-k} D_{T}$, we have $\left\{F_{j}^{0}\right\}(0,0) \subset L_{k}^{0}$ for all $k \geq j$ by Lemma 4.10. We conclude that $\left\{F_{j}^{0}\right\}(0,0) \subset L^{0}$, and since $j$ was arbitrary, the claim follows.

\section{The Necklace}

Let $F$ be a homeomorphism of the cylinder. Its lift, which will be denoted by the same letter, is a periodic map of the plane; we implement periodicity by requiring that $F$ commute with the backward rotation $R:(x, y) \mapsto(x-1, y)$. The direction of the $y$-axis will be called the vertical direction.

Assume now that the pair $(R, F)$ is asymptotically self-similar in the sense of Sect. 4 and that the corresponding fixed point satisfies the assumptions A1, A2, and A3. Then, according to Theorem 4.13 , there exists a compact connected invariant set for a certain iterated image of this pair under the renormalization operator. The question arises what this means for the homeomorphism $F$.

For a certain fixed $m \geq 1$, let $\left(U_{0}, T_{0}\right)$ denote the $m^{\text {th }}$ iterated image of the pair $(R, F)$ under the renormalization operator. We then have

$$
\left(U_{0}, T_{0}\right)=B\left(F_{m-1}, F_{m}\right) B^{-1},
$$

where $F_{m}$ denotes the $m^{\text {th }}$ Fibonacci string of the symbols $R$ and $F$, and where $B$ is a linear-diagonal coordinate change, namely an $m$-fold product of lineardiagonal single-step rescalings. Let $m$ be so large that the assumptions of Sect. 4 hold for the orbit of the pair $\left(U_{0}, T_{0}\right)$. Then, Theorem 4.13 gives us a compact connected pair $\left(M^{0}, N^{0}\right)$ that is invariant for the pair $\left(U_{0}, T_{0}\right)$. It follows that the pair $(M, N)=B^{-1}\left(M^{0}, N^{0}\right)$ is invariant for the pair $\left(F_{m-1}, F_{m}\right)$.

We put

$$
L=M \cup N
$$

The set $L$ has two disadvantages: first, for large values of $m$, it will by very tiny, since the inverse rescalings are contractions. Second, it is not invariant for the map $F$.

The aim of this section is to extend $L$ to a larger set that is invariant for $F$ and that, intuitively speaking, goes around the cylinder. This set will be called the necklace.

As a preparation, we derive two consequences of the results of Sect. 4: 
Lemma 5.1. Let $\left(U_{0}, T_{0}\right)$ be the $m^{\text {th }}$ iterated image of the pair $(R, F)$ under the renormalization operator, where $m$ is defined as before. Let $\left(M^{0}, N^{0}\right)$ be the invariant pair that Theorem 4.13 yields for $\left(U_{0}, T_{0}\right)$. Then the following relations hold:

$$
U_{0} N^{0} \cap N^{0} \neq \emptyset, \quad T_{0} U_{0}^{-1} N^{0} \cap M^{0} \neq \emptyset .
$$

Proof. By definition, the maps $U_{0}$ and $T_{0}$ are rescaled compositions of homeomorphisms of the plane, and it follows that $U_{0}$ and $T_{0}$ are homeomorphisms of the plane. Therefore, we no longer have to worry about domains of definition.

The first equation follows from the first equation of Lemma 4.2 by putting $k=0$ and going to the limit, observing that the sequence $\left(\overline{N_{j}^{o}}\right)$ is a decreasing sequence of compact sets.

The second one is obtained formally by putting $k=-1$ in the second equation of Lemma 4.2; for this to be valid, we have to restate the basic assumptions of Sect. 4 so that they hold for $k \geq-1$, which can be done without loss of generality. By going to the limit, we obtain the relation

$$
U_{-1} B_{0}^{-1} N^{0} \cap B_{0}^{-1} M^{0} \neq \emptyset \text {. }
$$

Since we have $U_{0}=B_{0} T_{-1} B_{0}^{-1}$ and $T_{0}=B_{0} U_{-1} T_{-1} B_{0}^{-1}$ by definition, we conclude that $B_{0} U_{-1} B_{0}^{-1}=T_{0} U_{0}^{-1}$. Since the relations $U_{-1} B_{0}^{-1} N^{0} \cap B_{0}^{-1} M^{0} \neq \emptyset$ and $B_{0} U_{-1} B_{0}^{-1} N^{0} \cap M^{0} \neq \emptyset$ are equivalent, this proves the assertion.

Now, we are ready to extend the invariant set. First, we make a slight change of notation: in order to get rid of the rescaling $B$, for the rest of this section we denote by $U$ the map $F_{m-1}$ and by $T$ the map $F_{m}$ (remember that $F_{m}$ is the $m^{\text {th }}$ Fibonacci string of the symbols $R$ and $F$ ). It has already been observed that the pair $(M, N)=B^{-1}\left(M_{0}, N_{0}\right)$ is invariant for $(U, T)$. By the above remark, the maps $U$ and $T$ are well defined on the whole plane. Lemma 5.1 translates into

\section{Lemma 5.2.}

$$
U N \cap N \neq \emptyset, \quad T U^{-1} N \cap M \neq \emptyset .
$$

We now define a finite sequence of pairs $\left(U_{\jmath}, T_{\jmath}\right)_{\jmath=0}^{m}$ by means of the following recursion: we put $U_{0}=U$ and $T_{0}=T$ and

$$
U_{\jmath+1}=T_{j} U_{j}^{-1}, \quad T_{\jmath+1}=U_{j}
$$

for $0 \leq j<m$.

Lemma 5.3. We have

$$
U_{j}=F_{m-j-1}, \quad T_{j}=F_{m-j}
$$

for $0 \leq j \leq m$.

Proof. For $j=0$, the assertion follows from the definition of $U$ and $T$.

Proceeding by induction, we obtain

$$
U_{j+1}=T_{j} U_{j}^{-1}=F_{m-\jmath} F_{m-j-1}^{-1}=F_{m-\jmath-2} F_{m-j-1} F_{m-\jmath-1}^{-1}=F_{m-\jmath-2}
$$

by the definition of the Fibonacci strings. Moreover, we have

$$
T_{j+1}=U_{j}=F_{m-\jmath-1} .
$$

The preceding lemma implies that

$$
U_{m}=R, \quad T_{m}=F ;
$$


therefore, at the end of our recursively defined sequence stands the original pair $(R, F)$.

In addition, we define a finite sequence of pairs $\left(M_{\jmath}, N_{\jmath}\right)_{\jmath=0}^{m}$ of sets by means of the following recursion: we put $M_{0}=M$ and $N_{0}=N$ and

$$
M_{j+1}=U_{\jmath} N_{\jmath}, \quad N_{j+1}=M_{j} \cup N_{\jmath}
$$

for $0 \leq j<m$.

Lemma 5.4. We have the following relations:

$$
U_{j} N_{j} \cap N_{j} \neq \emptyset, \quad T_{j} U_{j}^{-1} N_{\jmath} \cap M_{j} \neq \emptyset
$$

for $0 \leq j \leq m$.

Proof. For $j=0$, the claims are equivalent to the conclusions of Lemma 5.2.

Proceeding by induction, we obtain

$$
\begin{aligned}
U_{j+1} N_{\jmath+1} \cap N_{j+1} & =T_{\jmath} U_{j}^{-1}\left(M_{\jmath} \cup N_{j}\right) \cap\left(M_{\jmath} \cup N_{j}\right) \\
& \supset T_{\jmath} U_{j}^{-1} N_{j} \cap M_{j} \neq \emptyset
\end{aligned}
$$

and

$$
\begin{aligned}
T_{j+1} U_{\jmath+1}^{-1} N_{j+1} \cap M_{j+1} & =U_{\jmath} U_{\jmath} T_{j}^{-1}\left(M_{j} \cup N_{\jmath}\right) \cap U_{\jmath} N_{j} \\
& =U_{j}^{2} T_{j}^{-1}\left(\left(M_{\jmath} \cup N_{j}\right) \cap T_{\jmath} U_{j}^{-1} N_{j}\right) \\
& \supset U_{j}^{2} T_{j}^{-1}\left(M_{\jmath} \cap T_{j} U_{\jmath}^{-1} N_{j}\right) \neq \emptyset .
\end{aligned}
$$

Lemma 5.5. We have

$$
M_{\jmath} \cap N_{j} \neq \emptyset
$$

for $0 \leq j \leq m$.

Proof. For $j=0$, the claim follows from Lemma 4.3 by putting $k=0$ and passing to the limit. (The index $j$ there has a different meaning.)

Proceeding by induction, we obtain

$$
M_{j+1} \cap N_{\jmath+1}=U_{j} N_{j} \cap\left(M_{\jmath} \cup N_{j}\right) \supset U_{j} N_{j} \cap N_{\jmath} \neq \emptyset
$$

by Lemma 5.4 .

Lemma 5.6. The sets $M_{j}$ and $N_{j}$ are connected for $0 \leq j \leq m$.

Proof. For $j=0$, we have $M=B^{-1} M^{0}$ and $N=B^{-1} N^{0}$, where $B$ is a homeomorphism and $M^{0}$ and $N^{0}$ are connected. Assuming that $M_{j}$ and $N_{j}$ are connected, so is $M_{\jmath+1}$, because it is the continuous image of a connected set, and so is $N_{j+1}$, because it is an overlapping union of two connected sets.

Lemma 5.7. The sets $L_{j}=M_{j} \cup N_{j}$ are connected for $0 \leq j \leq m$.

Proof. The assertion follows from Lemma 5.5 and Lemma 5.6.

Lemma 5.8. The sequence $\left(L_{j}\right)$ is increasing.

Proof. The assertion follows directly from the definitions of $M_{\jmath+1}$ and $N_{j+1}$.

Lemma 5.9. The pairs $\left(M_{j}, N_{\jmath}\right)$ are invariant for $\left(U_{\jmath}, T_{\jmath}\right)$ for $0 \leq j \leq m$.

Proof. For $j=0$, the assertion is a consequence of the definition of $\left(M_{0}, N_{0}\right)$ and of Theorem 4.13. 
Proceeding by induction, we obtain

$$
U_{j+1} M_{j+1}=T_{j} U_{j}^{-1} U_{j} N_{j}=T_{j} N_{\jmath} \subset L_{j} \subset L_{\jmath+1}
$$

and

$$
T_{\jmath+1} N_{j+1}=U_{\jmath} M_{j} \cup U_{j} N_{\jmath} \subset L_{j} \cup M_{\jmath+1} \subset L_{j+1} .
$$

Of course, we are especailly interested in the pair $\left(M_{m}, N_{m}\right)$. We have shown that it is invariant for $(R, F)$ and that its union $L_{m}$ is connected. Moreover, being a finite union of compact sets, $L_{m}$ is compact. We have $R M_{m} \subset L_{m}$ by invariance; in particular, we have

$$
R L_{m} \cap L_{m} \neq \emptyset, \quad L_{m} \cap R^{-1} L_{m} \neq \emptyset,
$$

and it follows that the set

$$
\mathscr{B}=\bigcup_{n \in \mathbb{Z}} R^{n} L_{m}
$$

is connected. Since $L_{m}$ is compact, $\mathscr{L}$ is closed and bounded in the vertical direction from above and from below.

Lemma 5.10. We have

$$
F \mathscr{B} \subset \mathscr{L}
$$

Proof. Let $z$ be in $\mathscr{L}$. Then there exists a number $n \in \mathbb{Z}$ such that $w=R^{-n} z \in L_{m}$. If $w$ is in not in $N_{m}$, it is in $M_{m}$, and it follows that $R w \in L_{m}$. Since $L_{m}$ is compact, there is a natural number $l$ such that $R^{l} w \in L_{m}$ but $R^{l} w \notin M_{m}$. In any case, we have $R^{-n+l} z \in N_{m}$ with a natural number $l$. Applying $F$ to this point gives $F R^{-n+l} z \in L_{m}$. Now, since $F$ is a periodic map, it follows that $R^{-n+l} F z \in L_{m}$, and we have proved that $F z \in R^{n-l} L_{m} \subset \mathscr{L}$.

Definition 5.11. A set $\mathbb{L}$ is called invariant for a map $F$ if

$$
F \mathbb{L}=\mathbb{L} .
$$

Now we are ready to define the necklace set $\mathbb{L}$ :

$$
\mathbb{L}=\bigcap_{n \geq 0} F^{n} \mathscr{D}
$$

Because of $F \mathscr{L} \subset \mathscr{L}$, the necklace set thus defined is the limit set of a decreasing family of sets.

Lemma 5.12. The necklace set is invariant for $R$.

Proof. The set $\mathscr{L}$ is $R$-invariant by construction. For arbitrary non-negative integers $n$, we have

$$
R F^{n} \mathscr{L}=F^{n} R \mathscr{B}=F^{n} \mathscr{B},
$$

which shows that the sets $F^{n} \mathscr{L}$ are $R$-invariant. Therefore, the necklace set, being the intersection of $R$-invariant sets, is itself $R$-invariant.

Lemma 5.13. The necklace set is invariant for $F$.

Proof. Since $F \mathscr{B} \subset \mathscr{B}$, it follolws that $F \mathbb{L} \subset \mathbb{L}$. On the other hand we have

$$
F \mathbb{L}=\bigcap_{n \geq 0} F^{n+1} \mathscr{L}=\bigcap_{n \geq 1} F^{n} \mathscr{L} \supset \mathbb{L} .
$$


Lemma 5.14. The necklace set is non-empty, closed, connected, and vertically bounded.

Proof. Since the necklace set is $R$-invariant, it can be regarded as a set on the cylinder. Regarded as a set on the cylinder, it is the limit set of a decreasing family of non-empty compact sets. Therefore, the necklace set is non-empty.

Regarded as a set in the plane, the necklace set is the limit set of a decreasing family of closed, connected, and vertically bounded sets. Therefore it is itself closed, connected, and vertically bounded.

Definition 5.15. A subset of the plane is called separating if its complement has two vertically unbounded connected components. A subset of the cylinder is called separating if its complement has two unbounded connected components.

Lemma 5.16. Let $L$ be a subset of the plane that is closed, connected, vertically bounded, and $R$-invariant. Then $L$ is separating. Moreover, $L$ is the lift of a separating set on the cylinder.

Proof. Since $L$ is closed, the connected components of its complement are open.

Since $L$ is confined in a horizontal strip, its complement has at least one and at most two vertically unbounded components. Assume that there is just one vertically unbounded component. Choose a point $z_{1}$ below and a point $z_{2}$ above the strip confining $L$. By assumption, these points belong to the same open connected component of the complement of $L$. Therefore, there exists a path in the complement of $L$, joining $z_{1}$ to $z_{2}$. Now we add two unbounded vertical segments, one starting at the point $z_{2}$ and going upward, and the other one coming from below and ending at $z_{1}$. The resulting infinite path is contained in the complement of $L$, and, by Jordan's theorem, divides the plane into exactly two regions, namely into a left one and a right one. By $R$-invariance, the set $L$ intersects both regions. On the other hand, $L$ is contained in their union. This contradicts the connectedness of $L$.

The second claim follows from the first one.

We say that a homeomorphism of the cylinder is attracted by a fixed point if the pair $(R, F)$ is asymptotically self-similar in the sense of Sect.4. Summing up, we haved proved the following result:

Theorem 5.17. If a homeomorphism of the cylinder is attracted by a fixed piont that satisfies the assumptions $\mathrm{A} 1, \mathrm{~A} 2$, and $\mathrm{A} 3$, then it has a compact, connected, separating invariant set.

Proof. Theorem 4.13 yields a connected invariant pair for a certain iterated image of the pair $(R, F)$ under the renormalization operator. By means of the construction presented in this section, we build a necklace set out of this invariant pair. This necklace set, regarded as a subset of the cylinder, has the desired qualities.

\section{The Golden Curve}

In this section, we consider area-preserving, orientation-preserving, end-preserving $C^{1}$ twist diffeomorphisms of the cylinder that are attracted by the critical fixed point (or, for that matter, by any fixed point that satisfies the assumptions A1, A2, and A3.) 
For instance, the standard map, given by

$$
x^{\prime}=x+y^{\prime}, \quad y^{\prime}=y-\frac{\kappa}{2 \pi} \sin 2 \pi x
$$

with $\kappa$ being equal to the critical value $\kappa_{c} \approx 0.9716$ satisfies these assumptions.

Applying Theorem 5.17 yields a compact, connected, separating invariant set. We shall deduce from this the existence of an invariant golden circle, which is the graph of a Lipschitz function. The principal tool we are going to use is Birkhoff's theory on invariant curves for twist maps. Indeed, given Theorem 5.17, the existence of an invariant curve is an easy consequence of this theory. But rather than giving an abstract existence result, we shall show that the invariant curve is identical to the necklace set constructed in the last section.

In order to prove that this curve has the golden rotation number, we need some machinery that we are going to develop in Lemmas 6.3-6.8. These are not illuminating; I suggest that they be skipped on first reading.

Definition 6.1. The positive root of the equation

$$
x^{2}+x-1=0
$$

will be called the golden number and will be denoted by $\omega$.

Definition 6.2. Let $F$ be a periodic homeomorphism of the plane, i.e., a homeomorphism commuting with the backward rotation $R:(x, y) \mapsto(x-1, y)$. Let $\pi_{1}$ denote the projection onto the first coordinate direction. If for a point $z$ of the plane the limit

$$
\lim _{n \rightarrow \infty} \frac{\pi_{1} F^{n}(z)}{n}
$$

exists, it is called its rotation number.

In the following, we always assume that $F$ is an asymptotically self-similar homeomorphism in the sense of Sect. 4 and that the corresponding fixed point satisfies the assumptions A1, A2, and A3 stated in Sect. 1.

Of course, we should like the rotation number to be well defined on the set $\mathbb{L}$ and to be equal to the golden number $\omega$. But I was not able to prove this directly. Instead of that, we shall show that under the above assumptions, for each point $z \in \mathbb{L}$, there exist rational numbers $\frac{p}{q}$, arbitrarily close to the golden number $\omega$, such that

$$
\left|F^{q} R^{p} z\right| \leq c
$$

with a certain constant $c$. This implies that, if for a point $z \in \mathbb{L}$ the rotation number exists a priori, then it is equal to $\omega$; this will turn out to be enough for our purposes.

Lemma 6.3. Let $\Sigma$ act on pairs of natural numbers by

$$
\Sigma((p, q))=(p, q+p)
$$

and put $\left(p^{\prime}, q^{\prime}\right)=\Sigma((p, q))$. Then for arbitrary positive, $c$, the following implication holds:

$$
\left|\frac{p}{q}-\omega\right|<\frac{c}{q} \Rightarrow\left|\frac{p^{\prime}}{q^{\prime}}-\omega\right|<\frac{c}{q^{\prime}} .
$$


Proof. By a straightforward computation, we obtain

$$
\begin{aligned}
\left|\frac{q}{p+q}-\omega\right| & =\left|\frac{1}{1+\frac{p}{q}}-\frac{1}{1+\omega}\right| \\
& =\left|\omega-\frac{p}{q}\right| \frac{1}{1+\frac{p}{q}} \frac{1}{1+\omega}<\frac{c}{q} \frac{q}{p+q} \omega<\frac{c}{p+q} .
\end{aligned}
$$

Lemma 6.4. For any string $F$ of the symbols $U$ and $T$, we denote by $p$ the number of occurrences of the symbol $U$ within $F$, and by $q$ the number of occurrences of $T$; we then say that the string $F$ is characterized by the pair $(p, q)$.

a) Let $F$ be a Fibonacci tail (see Sect.2) different from the empty string. Then the following inequality holds:

$$
\left|\frac{p}{q}-\omega\right|<\frac{1}{q} .
$$

b) Let $F$ be any head of a Fibonacci string different from the empty string and from the string $U$. Then the same inequality holds.

Proof. We begin by proving the first statement. For the string $T$, we have $p=0$ and $q=1$; for the string $T U$, we have $p=1$ and $q=1$. Since $|0-\omega|<1$ and $|1-\omega|<1$ (observe that $\omega \approx 0.618$ ), the claim is true.

Assume that the claim has been proved for strings of length up to $l+1$ with $l \geq 0$. Let $F$ be the (unique) Fibonacci tail of length $l+2$. According to Lemma 2.8, we have either $F=\Sigma(G)$ or $F=T \Sigma^{2}(G)$, where $G$ is a Fibonacci tail shorter than $F$. Our assumptions rule out the case that $G$ is empty. Let $G$ be characterized by the pair $(p, q)$. If $F=\Sigma(G)$, then $F$ is characterized by the pair $\Sigma((p, q))$, and by Lemma 6.3 and the inductive assumption, we are done.

If $F=T \Sigma^{2}(G)$, then $F$ is characterized by the numbers $(p+q, p+2 q+1)$. Let us perform a preliminary computation:

$$
\frac{p+q}{p+2 q+1}=\frac{1}{1+\frac{q+1}{p+q}}=\frac{1}{1+\frac{1}{1+\frac{p-1}{q+1}}}
$$

Putting $\beta=\frac{p-1}{q+1}$, we obtain

$$
\begin{aligned}
\left|\frac{p+q}{p+2 q+1}-\omega\right| & =\left|\frac{1}{1+\frac{1}{1+\beta}}-\frac{1}{1+\frac{1}{1+\omega}}\right|=\left|\frac{1}{1+\omega}-\frac{1}{1+\beta}\right| \frac{p+q}{p+2 q+1} \omega \\
& =|\beta-\omega| \omega \frac{q+1}{p+q} \frac{p+q}{p+2 q+1} \omega=|\beta-\omega| \omega^{2} \frac{q+1}{p+2 q+1} .
\end{aligned}
$$

From the inductive assumption

$$
-1<q \omega-p<1
$$

it follows that

$$
\omega<(q+1) \omega-(p-1)<2+\omega,
$$


which implies that

$$
|\omega-\beta|<\frac{2+\omega}{q+1}
$$

Making use of the identity $(2+\omega) \omega^{2}=1$, we end up with

$$
\left|\frac{p+q}{p+2 q+1}-\omega\right|<\frac{1}{p+2 q+1},
$$

which terminates the proof of a).

The proof of the second statement is analogous. We give it only for the sake of completeness.

Instead of Lemma 2.8 we use a direct consequence thereof, namely the formula

$$
\left[F_{\jmath+2}\right]=\Sigma\left(\left[F_{j+1}\right]\right) \cup\left(\Sigma^{2}\left(\left[F_{\jmath}\right]\right)-T\right),
$$

where $\left[F_{j}\right]$ denotes the set of heads of the $j^{\text {th }}$ Fibonacci string, not including the empty one, and where the formal subtraction of $T$ means taking away the symbol $T$ from the end of the string. The heads of the Fibonacci strings will be called Fibonacci heads.

Let us first deduce this formula from Lemma 2.8. To this end, we take any $\Delta \in\left[F_{j+2}\right]$. By definition, we have $F_{j+2}=\Delta F$ with a string $F \in\left\{F_{j+2}\right\}$. According to Lemma 2.8, we have either $F=\Sigma(G)$ or $F=T \Sigma^{2}(G)$ with a string $G$ in $\left\{F_{j+1}\right\}$, respectively in $\left\{F_{j}\right\}$.

In the first case, we have

$$
F_{j+2}=\Sigma\left(F_{j+1}\right)=\Delta \Sigma(G)
$$

and from the formula $\Sigma\left(F_{j+1}-G\right)=\Sigma\left(F_{j+1}\right)-\Sigma(G)$, it follows that $\Delta=$ $\Sigma\left(F_{\jmath+1}-G\right)$. In this case, therefore, we have $\Delta \in \Sigma\left(\left[F_{j+1}\right]\right)$.

In the second case, we have

$$
F_{j+2}=\Sigma^{2}\left(F_{j}\right)=\Delta T \Sigma^{2}(G),
$$

and from the formula $\Sigma^{2}\left(F_{j}-G\right)=\Sigma^{2}\left(F_{j}\right)-\Sigma^{2}(G)$, it follows that $\Delta T=$ $\Sigma^{2}\left(F_{j}-G\right)$. In this case, therefore, we have $\Delta \in \Sigma^{2}\left(\left[F_{j}\right]\right)-T$.

Now, we come back to the second half of Lemma 6.4. For the strings $T, T U$, and $U T$, the claims are true. (Remember that the string $U$ is ruled out by assumption.)

Assume that the claim has been proved for strings of length up to $l+1$ with $l \geq 0$. Let $F$ be a Fibonacci head of length $l+2$. According to the above formula, we have either $F=\Sigma(G)$ or $F=\Sigma^{2}(G)-T$, where $G$ is a Fibonacci head shorter than $F$. Our assumptions rule out the case that $G$ is empty. Since $\Sigma(U)=T$ and $\Sigma^{2}(U)-T=U$, the case $G=U$ is ruled out as well.

Let $G$ be characterized by the pair $(p, q)$. If $F=\Sigma(G)$, then $F$ is characterized by the pair $\Sigma((p, q))$, and by Lemma 6.3 and the inductive assumption, we are done.

If $F=\Sigma^{2}(G)-T$, then $F$ is characterized by the numbers $(p+q, p+2 q-1)$.

Let us perform a preliminary computation:

$$
\frac{p+q}{p+2 q-1}=\frac{1}{1+\frac{q-1}{p+q}}=\frac{1}{1+\frac{1}{1+\frac{p+1}{q-1}}}
$$


The Fibonacci strings of length greater than 2 alternatingly start with the sequences TUT and UTT. Therefore, we have $q>1$, and the last equation makes sense.

Putting $\beta=\frac{p+1}{q-1}$, we obtain

$$
\begin{aligned}
\left|\frac{p+q}{p+2 q-1}-\omega\right| & =\left|\frac{1}{1+\frac{1}{1+\beta}}-\frac{1}{1+\frac{1}{1+\omega}}\right|=\left|\frac{1}{1+\omega}-\frac{1}{1+\beta}\right| \frac{p+q}{p+2 q-1} \omega \\
& =|\beta-\omega| \omega \frac{q-1}{p+q} \frac{p+q}{p+2 q-1} \omega=|\beta-\omega| \omega^{2} \frac{q-1}{p+2 q-1} .
\end{aligned}
$$

From the inductive assumption

$$
-1<q \omega-p<1
$$

it follows that

$$
-2-\omega<(q-1) \omega-(p+1)<-\omega,
$$

which implies that

$$
|\omega-\beta|<\frac{2+\omega}{q-1}
$$

Making use of the identity $(2+\omega) \omega^{2}=1$, we end up with

$$
\left|\frac{p+q}{p+2 q-1}-\omega\right|<\frac{1}{p+2 q-1},
$$

which terminates the proof of $b)$.

Lemma 6.5. Let $\left(p_{j}, q_{j}\right)$ and $(p, q)$ be two pairs of natural numbers. If

$$
\left|\frac{p_{j}}{q_{j}}-\omega\right|<\frac{1}{q_{j}} \quad \text { and either } \quad\left|\frac{p}{q}-\omega\right|<\frac{1}{q} \quad \text { or } \quad q=0, q=1,
$$

it follows that

$$
\left|\frac{p+p_{j}}{q+q_{j}}-\omega\right|<\frac{2}{q+q_{j}} .
$$

Proof. For $q \neq 0$, making use of the identity

$$
\frac{p+p_{j}}{q+q_{\jmath}}=\frac{p}{q} \frac{q}{q+q_{\jmath}}+\frac{p_{j}}{q_{\jmath}} \frac{q_{j}}{q+q_{j}},
$$

we obtain

$$
\left|\frac{p+p_{j}}{q+q_{j}}-\omega\right| \leq\left|\frac{p}{q}-\omega\right| \frac{q}{q+q_{j}}+\left|\frac{p_{\jmath}}{q_{\jmath}}-\omega\right| \frac{q_{j}}{q+q_{\jmath}} \leq \frac{2}{q+q_{j}} .
$$

For $q=0$ and $p=1$, we obtain

$$
\left|\frac{p_{j}+1}{q_{\jmath}}-\omega\right| \leq\left|\frac{p_{\jmath}}{q_{\jmath}}-\omega\right|+\frac{1}{q_{j}}<\frac{2}{q_{\jmath}} .
$$


Lemma 6.6. (We are using the notations of Sect.4.) Assume that the maps $U_{0}$ and $T_{0}$ are globally defined and that they commute. Then there exists a constant $\tilde{c}$ such that for each point $z \in L^{0}$, there exist arbitrarily high natural numbers $p$ and $q$ such that

$$
\left|\frac{p}{q}-\omega\right|<\frac{2}{q}, \quad\left|U_{0}^{p} T_{0}^{q} z\right| \leq \tilde{c} .
$$

Proof. For the notation, see the proof of Lemma 4.12. We define $\tilde{c}$ to be a bound for the sets $B_{1}^{-\jmath} T_{\jmath} D_{T}$. (Remember that the sets $T_{j} D_{T}$ are uniformly bounded and that inverse rescalings are contractions.) Fix $j$ and choose $k \geq j$ such that $B_{1}^{-k}\left(U_{k} D_{U} \cup T_{k} D_{T}\right) \subset B_{1}^{-j} D_{T}$.

Let $z$ be an arbitrary point of $L^{0}$. In particular, $z$ lies in $L_{k}^{0}$, and by Lemma 4.10, we have either $z \in F B_{1}^{-k} D_{T}$ or $z \in G B_{1}^{-k} D_{U}$, where $F$ and $G$ are tails of the Fibonacci strings $F_{k}^{0}$ or $F_{k-1}^{0}$ respectively. Assume for instance that $z \in F B_{1}^{-k} D_{T}$. (For $z \in G B_{1}^{-k} D_{U}$, the reasoning is completely analogous.)

Let $\Delta$ be the difference string to the Fibonacci string $F_{k}^{0}$. Then $\Delta z \in F_{k}^{0} B_{1}^{-k} D_{T}=$ $B_{1}^{-k} B_{1}^{k} F_{k}^{0} B_{1}^{-k} D_{T}=B_{1}^{-k} F_{0}^{k} D_{T}=B_{1}^{-k} T_{k} D_{T}$ by Lemma 4.8. By the definition of $k$, it follows that $\Delta z \in B_{1}^{-j} D_{T}$. Now, we apply $F_{j}^{0}$ to this point, and by the same reasoning as before, it follows that $F_{j}^{0} \Delta z \in B_{\jmath}^{-j} T_{j} D_{T}$. By the definition of $\tilde{c}$, it follows that

$$
\left|F_{j}^{0} \Delta z\right| \leq \tilde{c}
$$

By commutativity, the map $F_{j}^{0} \Delta$ can be written as $U_{0}^{p} T_{0}^{q}$, where the numbers $p$ and $q$ characterize the string $F_{j}^{0}$ (see Lemma 6.4). It follows that

$$
\left|U_{0}^{p} T_{0}^{q} z\right| \leq \tilde{c} .
$$

If $\Delta \neq U_{0}$, since Lemma 6.4 applies to both $F_{j}^{0}$ and $\Delta$, we use Lemma 6.5 to conclude that $\left|\frac{p}{q}-\omega\right|<\frac{2}{q}$. For $\Delta=U_{0}$, the second case of Lemma 6.5 gives the same answer.

Finally, by choosing $j$ high enough, we can make $q$ arbitrarily high.

Lemma 6.7. (Now we are using the notations of Sect.5.) Assume that the maps $U$ and $T$ are globally defined and that they commute. Then there exists a constant $c$ such that for each point $z \in L_{m}$, there exist arbitrarily high natural numbers $p$ and $q$ such that

$$
\left|\frac{p}{q}-\omega\right|<\frac{2}{q}, \quad\left|U^{p} T^{q} z\right| \leq c .
$$

Proof. Recall the notations of Sect. 5: we have

$$
L=B^{-1} L^{0}
$$

(for the definition of $L^{0}$, see Sect. 4), where $B$ is an $m$-fold product of linear-diagonal single-step rescalings. Furthermore, we have

$$
U=B^{-1} U_{0} B=F_{m-1}, \quad T=B^{-1} T_{0} B=F_{m},
$$

where $F_{j}$ is the $j^{\text {th }}$ Fibonacci string of the symbols $R$ and $F$.

Therefore, Lemma 6.6 stays valid with $L$ replacing $L^{0}$, with $(U, T)$ replacing $\left(U_{0}, T_{0}\right)$, and with $\left\|B^{-1}\right\| \tilde{c}$ replacing $\tilde{c}$. 
Looking again at the construction of the set $L_{m}$ in Sect. 5, we see that $L_{m}$ is a finite union of sets of the form $A M$ and $A L$, where $A$ is a homeomorphism of the plane that commutes both with $U$ and with $T$. We define $c$ to be a simultaneous bound for the (finitely many) maps $A$ on the disk of radius $\tilde{c}$, where $\tilde{c}$ is the constant from Lemma 6.6.

Let $z$ be an arbitrary point of $L_{m}$. Then we have $z=A w$, where $A$ is a homeomorphism of the plane and where $w$ is an element of $L$. For arbitrary natural numbers $p$ and $q$, we have

$$
U^{q} T^{p} z=U^{p} T^{q} A w=A U^{p} T^{q} w
$$

and the claim follows from Lemma 6.6 and the definition of $c$.

Lemma 6.8. There exists a constant $c$ such that for each point $z \in \mathbb{L}$ and for each positive $\varepsilon$, there exist natural numbers $p$ and $q$ such that

$$
\left|\frac{p}{q}-\omega\right|<\varepsilon, \quad\left|F^{q} R^{p} z\right| \leq c .
$$

Proof. We define $c$ to be the constant given by Lemma 6.7 .

Take any $z \in \mathbb{L}$. In particular, we have $z \in \mathscr{S}$. It follows that there exists an integer $n$ such that $R^{n} z \in L_{m}$. Making use of Lemma 6.7, we choose natural numbers $a$ and $b$ such that $\frac{|n|+2}{b}<\varepsilon,\left|\frac{a}{b}-\omega\right|<\frac{2}{b}$, and

$$
\left|U^{a} T^{b} R^{n} z\right| \leq c
$$

We put $\left(p^{\prime}, q^{\prime}\right)=\Sigma^{m}(a, b)$. Remember that $U=F_{m-1}$ and $T=F_{m}$; therefore, the above inequality can be rewritten as

$$
\left|R^{p} F^{q} z\right| \leq c
$$
where $p=p^{\prime}+n$ and $q=q^{\prime}$. Moreover, Lemma 6.5 yields $\left|\frac{p^{\prime}}{q^{\prime}}-\omega\right|<\frac{2}{q^{\prime}}$, and from
this we obtain

$$
\left|\frac{p}{q}-\omega\right| \leq\left|\frac{p^{\prime}}{q^{\prime}}-\omega\right|+\frac{|n|}{q^{\prime}}<\frac{2+|n|}{q^{\prime}} \leq \frac{2+|n|}{b}<\varepsilon .
$$

Theorem 6.9. Let $F$ be an area-preserving, orientation-preserving, end-preserving $C^{1}$ twist diffeomorphism of the cylinder. If $F$ is attracted by any fixed point that satisfies the assumptions $\mathrm{A} 1, \mathrm{~A} 2$, and $\mathrm{A} 3$, then $F$ has an invariant golden circle that is the graph of a Lipschitz function.

Proof. Theorem 5.17 yields a compact, connected, separating invariant set for $F$, which we again denote by $\mathbb{L}$.

We are going to apply Birkhoff's theorem (see [4, p.303]). Let $U$ denote the connected component of the complement of $\mathbb{L}$ that contains the lower end of the cylinder. Since $F$ is a homeomorphism that preserves the ends of the cylinder, and since $L$ is invariant for $F$, it follows that $U$ is invariant for $F$. Because $\mathbb{L}$ is separating and bounded from above and from below, the set $U$ satisfies the assumptions of Birkoff's theorem, and it follows that its boundary is the graph of a Lipschitz curve. This curve is, of course, invariant for $F$.

Moreover, this curve is contained in the set $\mathbb{L}$. Since on an invariant curve, the rotation number is well defined, it follows from Lemma 6.8 that the rotation number on the invariant curve is equal to the golden number. 
Now, we turn the cylinder upside down and repeat these arguments. This gives a second golden curve, corresponding to the boundary of the open connected component sitting above $\mathbb{L}$.

But there is at most one invariant circle with a given irrational rotation number (see [1, Proposition I.5.7.1.]), and it follows that the two curves coincide. Since $\mathbb{L}$ is sandwiched between the two, $\mathbb{L}$ itself must be an invariant circle.

Since, in particular, the critical fixed point satisfies the assumptions A1, A2, and A3, Theorem 6.9 guarantees the existence of an invariant golden circle for any (areapreserving, orientation-preserving, end-preserving $C^{1}$ ) twist diffeomorphism in its domain of attraction. This curve is called the critical golden curve.

\section{Ordering the Patches}

Recall from Lemma 2.5 the following representation of the sets $L_{j}$ :

$$
L_{\jmath}=\left\{F_{j}\right\} B^{-j} D_{T} \cup\left\{F_{j-1}\right\} B^{-j} D_{U}, \quad j \geq 0 .
$$

The open sets appearing on the right-hand side are called Fibonacci patches or simply patches. The patches with a certain fixed value of $j$ are said to belong to the $j^{\text {th }}$ generation.

Now, we impose an additional assumption on the fixed point:

A4. The map $T B^{-2}$ is a uniform contraction on $D_{U} \cup D_{T}$.

(Again, this assumption can be justified numerically for the critical fixed point. For the simple fixed point, it is easy to verify by a direct calculation.) Looking at Lemma 1.5, we see that the maximal diameter of the patches decreases exponentially with increasing generation. It is a simple property of the Fibonacci patches that each patch of the form $F B^{-j} D_{U}$ intersects one of the form $G B^{-j} D_{T}$. (We shall prove stronger statements later on.) Since all the patches of the latter form contain a point of the orbit of $(0,0)$, it immediately follows that the orbit of $(0,0)$ is dense in the limit set. Therefore, the invariant circle constructed in the previous section is transitive.

But we can do better: by delving deeper into the combinatorics of the Fibonacci patches, we shall define a strict order on the patches of the same generation such that - only neighboring patches overlap, and that

- it is refined by successive generations.

Using this order as a tool, we shall be able to dispense with the Birkhoff theory in the construction of an invariant curve. In addition, this approach yields a homeomorphism that conjugates the induced map on the curve to a rigid rotation. Moreover, the conjugator turns out to be Hölder continuous with an exponent that is determined by the rescaling $B$.

For clarity, we shall be working only with the fixed point. The difficulties in carrying the argumentation over to the case of asymptotic self-similarity are merely notational (see appendix). The presentation is largely independent from the first part of this paper; in fact, only Lemma 1.5, Lemma 2.2, and Lemma 2.6 will be used.

The first aim of this section is to define two special relationships among the Fibonacci patches: a descendence and a succession. (Figure 6 is a schematic picture of (part of) the network that will arise. The horizontal arrows visualize the succession, the vertical ones the descendence.) The idea is that descendence implies set inclusion, and that successive patches overlap. More concretely: each patch with $j \geq 1$ will be 


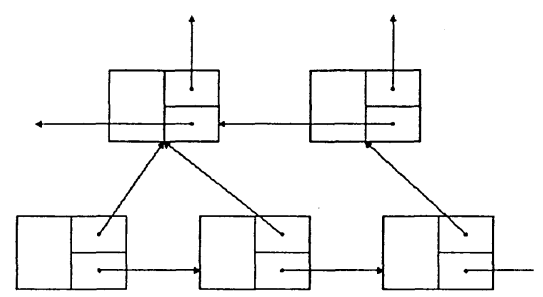

Fig. 6. Descendence and succession

shown to descend from exactly one parent, to which it belongs as a subset. Moreover, two patches of the same generation will be shown to overlap if and only if one is the successor of the other.

These two relationships will turn out to be compatible with a certain strict order on the patches of the same generation, that we are going to construct beforehand.

Let us start by defining an order on the Fibonacci tails. We do this by defining a injective map $\varrho$ from the space of Fibonacci tails into the real axis.

Definition 7.1. Let $\omega$ be the golden number. For any Fibonacci tail $F$, we denote by $q$ the number of occurrences of the symbol $T$ within $F$ and by $p$ the number of occurrences of $U$. Then the map $\varrho$ is defined by

$$
\varrho(F)=\omega q-p .
$$

This map is injective, $\omega$ being an irrational number.

Definition 7.2. A Fibonacci tail $F$ is said to be smaller than a Fibonacci tail $G$ if and only if $\varrho(F)<\varrho(G)$. Formally:

$$
F<G \Leftrightarrow \varrho(F)<\varrho(G) .
$$

Having defined an order on the Fibonacci tails, we go on to define an order on the patches of the same generation. A priori, this order will have nothing to do with the geometric shapes and positions of the patches; it will take into account only combinatorial properties of the patches. In order to emphasize this, let us introduce the patch descriptors:

Definition 7.3. A patch descriptor is an object consisting of a non-negative integer $j$, denoting what we call its generation, a Fibonacci tail $F$, and a tag that is either the symbol $U$ or the symbol $T . F$ is required to be a member of $\left\{F_{j-1}\right\}$ if the tag is equal to $U$, and a member of $\left\{F_{j}\right\}$ otherwise.

I am tempted to write down a $C$-style data structure for the patch descriptors:

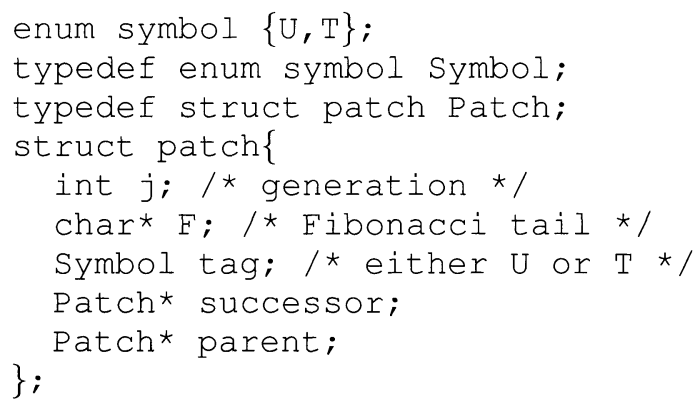


The variables successor and parent are reserved for later use, namely for defining the succession and the descendence.

The patch descriptors are in a one-to-one correspondence with the patches. For simplicity, we no longer distinguish in language between patches and patch descriptors. They will be denoted by $F B^{-j} D_{U}$ and $F B^{-j} D_{T}$ respectively.

Definition 7.4. Let $P$ and $Q$ be two different patches of the same generation. If their Fibonacci tails are different, $P$ is said to be smaller than $Q$ if and only if the Fibonacci tail of $P$ is smaller than the Fibonacci tail of $Q$. If their Fibonacci tails are identical, then we distinguish between even and odd values of the generation $j$ : for even $j$, the patch labeled by $T$ is smaller, for odd $j$, the patch labeled by $U$ is smaller.

Having ordered the patches of the same generation, we are going to define the succession among them. For this purpose, we need two elementary results on Fibonacci strings. Let us denote by $\hat{F}_{j}$ the string of maximal length within $\left\{F_{j}\right\}$.

Lemma 7.5. Let the formal subtraction $F-G$ denote the operation of removing the string $G$ from right end of the string $F$, provided of course that $G$ is contained in $F$. For $j \geq 2$, we have

$$
F_{j}-F_{j-2}= \begin{cases}T U F, & \text { for even } j \\ U T F, & \text { otherwise, }\end{cases}
$$

where $F$ is a Fibonacci teil in $\left\{F_{j-1}\right\}$ different from $\hat{F}_{j-1}$. (Indeed, it follows from a simple consideration of string lengths that the length of $F$ is one below the length of $\hat{F}_{j-1}$.)

Proof. For $j=2$, we have $F_{2}-F_{0}=T U T-T=T U=T U \diamond$, which is OK, since the empty string $\diamond$ is contained in $\left\{F_{1}\right\}$; for $j=3$, we have $F_{3}-F_{1}=$ $U T T U T-U T=U T T$, which is $\mathrm{OK}$, since the string $T$ is contained in $\left\{F_{2}\right\}$.

For any two strings $A$ and $B$, the equation $\Sigma(A B)=\Sigma(A) \Sigma(B)$ implies $\Sigma(A B)-\Sigma(B)=\Sigma(A)=\Sigma(A B-B)$. Making use of the latter formula, we obtain inductively

$$
\begin{aligned}
F_{j}-F_{j-2} & =\Sigma\left(F_{j-1}\right)-\Sigma\left(F_{j-3}\right) \\
& =\Sigma\left(F_{j-1}-F_{j-3}\right) \\
& = \begin{cases}\Sigma(U T F)=\operatorname{TUT} \Sigma(F), & \text { for even } j \\
\Sigma(T U F)=U T T \Sigma(F), & \text { otherwise }\end{cases}
\end{aligned}
$$

with a string $F \in\left\{F_{j-2}\right\}$ different from $\hat{F}_{j-2}$. Therefore, $\Sigma(F) \in\left\{F_{j-1}\right\}$ differs from $\hat{F}_{j-1}$. Lemma 2.6 tells us that the next symbol of $\Sigma(F)$ is $T$. It follows that $T \Sigma(F) \in\left\{F_{j-1}\right\}$; again, a simple consideration of string lengths shows that $T \Sigma(F)$ is different from $\hat{F}_{j-1}$.

Lemma 7.6. We have

$$
\varrho\left(F_{j}\right)=(-1)^{j} \omega^{j+1}
$$

for $j \geq 0$.

Proof. For $j=0$, we obtain $\varrho\left(F_{0}\right)=\varrho(T)=\omega$, which is OK.

The general claim follows inductively by making use of the identity

$$
\frac{\varrho\left(F_{j+1}\right)}{\varrho\left(F_{j}\right)}=-\omega
$$


which is verified by a direct calculation:

$$
\frac{\varrho\left(F_{j+1}\right)}{\varrho\left(F_{j}\right)}+\omega=\frac{\left(q_{j}+p_{j}\right) \omega-q_{j}}{q_{\jmath} \omega-p_{j}}+\omega=\frac{q_{j}\left(\omega^{2}+\omega-1\right)}{q_{\jmath} \omega-p_{\jmath}}=0 .
$$

Lemma 7.7. The sequence $\left(\varrho\left(\hat{F}_{2 j}\right)\right)$ decreases, and the sequence $\left(\varrho\left(\hat{F}_{2 \jmath+1}\right)\right)$ increases, and we have

$$
\lim _{j \rightarrow \infty} \varrho\left(\hat{F}_{2 j}\right)=-\omega \text { and } \lim _{j \rightarrow \infty} \varrho\left(\hat{F}_{2 \jmath+1}\right)=1 .
$$

Proof. Since by Lemma 7.5, the even Fibonacci strings start with $T$ and the odd ones with $U$, the claim follows from Lemma 7.6 and from Definition 7.1.

Lemma 7.5 implies: for any Fibonacci tail in $\left\{F_{j}\right\}$, except for the string of maximal length, removing $F_{j-2}$ from its right end yields a Fibonacci tail in $\left\{F_{j-1}\right\}$. Using this property, we now define the succession $\sigma$ among the patches of the same generation:

Definition 7.8. Each patch $P$ of the $j^{\text {th }}$ generation, except for the patch

$$
\hat{F}_{j} B^{-\jmath} D_{T},
$$

has a unique successor in the same generation, denoted by $\sigma(P)$, that is defined by

$$
\begin{aligned}
& \sigma\left(F B^{-j} D_{U}\right)=F B^{-j} D_{T} \\
& \sigma\left(F B^{-j} D_{T}\right)= \begin{cases}F F_{\jmath-1} B^{-j} D_{T}, & \text { if } F \in\left\{F_{j-2}\right\} \\
\left(F-F_{j-2}\right) B^{-j} D_{U}, & \text { if } F \in\left\{F_{\jmath}\right\} \backslash\left\{F_{\jmath-2}\right\} \text { and } F \neq \hat{F}_{\jmath} .\end{cases}
\end{aligned}
$$

(Do not confuse this succession with the succession given by increasing string length, which was used in Sect. 2.)

Because of Lemma 7.5, the successor is well defined. Moreover, it follows that $\hat{F}_{j-1} B^{-j} D_{U}$ is not the successor of any patch, because $F-F_{j-2}$ always differs from $\hat{F}_{j-1}$.

The significance of the succession lies in the following two results:

Lemma 7.9. With respect to the above defined order on the patches, the map $\sigma$ is decreasing for even $j$ and increasing for odd $j$.

Proof. By Definition 7.3, $F B^{-\jmath} D_{U}$ is smaller or greater than $F B^{-j} D_{T}$, according to whether $j$ is odd or even. Moreover, by Lemma 7.6, we have

$$
\varrho\left(F_{j-1}\right) \begin{cases}>0, & \text { for odd } j \\ <0, & \text { otherwise }\end{cases}
$$

Therefore, removing $F_{j-2}$ changes $\varrho$ into the same direction as adding $F_{j-1}$, namely into the positive one for odd values of $j$, and into the negative one for even values of $j$.

The succession makes the patches of the same generation a collection of singly linked lists. Since there is a numerical function, namely $\varrho$, that either increases or decreases along each list, there can be no loops, and it follows that there is just one list.

As noted above, the patch $\hat{F}_{j-1} B^{-j} D_{U}$ does not have a predecessor. Starting with this patch, the succession exhausts the whole generation. The last patch is $\hat{F}_{j} B^{-\jmath} D_{T}$, because it has no successor. The first and the last patch will be called boundary patches. 
By Lemma 7.9, the order defined by the succession either coincides with the order given by $\varrho$ or is opposite to it, depending on whether $j$ is odd or even.

Two patches of the same generation are called neighbors if and only if one of them is the successor of the other. The smaller neighbor will sometimes be called the left neighbor, and the larger one the right neighbor.

For the following lemma, we need our standard connectedness assumptions from Sect. 1.

Lemma 7.10 If two patches of the same generation are neighbors, then they overlap as sets.

Proof. According to Definition 7.8 there are three possible cases, each of which is reduced to one of the assumptions. Apart from Lemma 2.2, the reductions use the fact that the maps $U, T$, and $B$ are homeomorphisms.

First case:

$$
F B^{-j} D_{U} \cap F B^{-\jmath} D_{T} \neq \emptyset \Leftrightarrow D_{U} \cap D_{T} \neq \emptyset .
$$

Second case:

$$
\begin{aligned}
F B^{-j} D_{T} \cup F F_{j-1} B^{-j} D_{T} \neq \emptyset & \Leftrightarrow B^{-j} D_{T} \cap F_{j-1} B^{-j} D_{T} \neq \emptyset \\
& \Leftrightarrow D_{T} \cap U D_{T} \neq \emptyset .
\end{aligned}
$$

(Remember: $U$ has been continued to $D_{U} \cup D_{T}$, see Sect.1.)

Third case:

$$
\begin{aligned}
F F_{j-2} B^{-j} D_{T} \cap F B^{-\jmath} D_{U} \neq \emptyset & \Leftrightarrow F_{j-2} B^{-j} D_{T} \cap B^{-\jmath} D_{U} \neq \emptyset \\
& \Leftrightarrow U B^{-1} D_{T} \cap B^{-1} D_{U} \neq \emptyset .
\end{aligned}
$$

Now we define the descendence of the patches:

Definition 7.11. Each patch $P$ that does not belong to the zeroth generation has a unique parent, denoted by $\pi(P)$, that is defined by

$$
\begin{aligned}
& \pi\left(F B^{-(j+1)} D_{U}\right)=F B^{-j} D_{T}, \\
& \pi\left(F B^{-(j+1)} D_{T}\right)= \begin{cases}F B^{-j} D_{T}, & \text { for } F \in\left\{F_{j}\right\} \\
\left(F-F_{j}\right) B^{-j} D_{U}, & \text { for } F \in\left\{F_{j-1}\right\} F_{j} .\end{cases}
\end{aligned}
$$

The equation $F_{j+1}=F_{j-1} F_{j}$ implies $\left\{F_{j+1}\right\}=\left\{F_{j-1}\right\} F_{j} \cup\left\{F_{j}\right\}$. Therefore, the descendence is well defined.

Reading this definition backward, we see that each patch tagged by $T$ gives rise to exactly two children, whereas a patch tagged by $U$ has exactly one child. More precisely: the (direct) progeny of the patch $F B^{-j} D_{T}$ consists of the two patches $F B^{-(j+1)} D_{U}$ and $F B^{-(j+1)} D_{T}$. The unique child of the patch $F B^{-j} D_{U}$ is $F F_{j} B^{-(j+1)} D_{T}$ (note that $F \in\left\{F_{j-1}\right\}$ implies $F F_{j} \in\left\{F_{j+1}\right\}$ ).

The main significance of the descendence lies in the following result, the proof of which requires our standard assumptions on domain extension:

Lemma 7.12 The closure of each patch with $j \geq 1$ is contained in its parent.

Proof. According to Definition 7.11, there are three cases to distinguish, each of which is reduced to one of the assumptions. Apart from Lemma 2.2, the reductions use the fact that the maps $U, T$, and $B$ are homeomorphisms.

First case:

$$
F B^{-(\jmath+1)} \overline{D_{U}} \subset F B^{-\jmath} D_{T} \Leftrightarrow B^{-1} \overline{D_{U}} \subset D_{T} .
$$


Second case:

$$
F B^{-(j+1)} \overline{D_{T}} \subset F B^{-j} D_{T} \Leftrightarrow B^{-1} \overline{D_{T}} \subset D_{T} .
$$

Third case:

$$
F F_{j} B^{-(j+1)} \overline{D_{T}} \subset F B^{-j} D_{U} \Leftrightarrow T B^{-1} \overline{D_{T}} \subset D_{U} .
$$

Thus far, we have given an alternative proof for the facts that the sequence $\left(L_{\jmath}\right)$ is decreasing and that the sets $L_{\jmath}$ are connected.

Now we show that the descendence refines the order of the patches:

Lemma 7.13. Let $P$ and $Q$ be two patches of the same generation. If $P$ is smaller than $Q$, then each descendent of $P$ is smaller than each descendent of $Q$ of the same generation.

Proof. It is enough to prove the claim in the case that $P$ and $Q$ are neighbors and that the descendents are children. Assume that the generation of $P$ and $Q$ is odd. Then the succession ties in with the order. (The proof for even values of $j$ is entirely analogous.) According to Definition 7.8, there are three cases to distinguish:

First case: $P=F B^{-j} D_{U}<Q=F B^{-j} D_{T}$. Here, $F$ is in $\left\{F_{j-1}\right\}$. The unique child of $P$ is $F F_{j} B^{-(j+1)} D_{T}$. The progeny of $Q$ consists of two neighboring patches that are ordered as follows:

$$
F B^{-(j+1)} D_{T}<F B^{-(j+1)} D_{U} .
$$

(In the generation $j+1$, the succession is opposite to the order.) It is enough to show that $F F_{j} B^{-(j+1)} D_{T}<F B^{-(j+1)} D_{T}$. This in turn follows from the facts that the successor of $F B^{-(j+1)} D_{T}$ is $F F_{j} B^{-(\jmath+1)} D_{T}$ (remember that $F \in\left\{F_{\jmath-1}\right\}$ ) and that the generation $j+1$ is even.

Second case: $P=F F_{j-2} B^{-j} D_{T}<Q=F B^{-j} D_{U}$. (This case implies $j>1$.) Here, $F$ is in $\left\{F_{j-1}\right\}$ and $F F_{j-2}$ is different from $\hat{F}_{j}$. The progeny of $P$ consists of two neighboring patches that are ordered as follows:

$$
F F_{j-2} B^{-(j+1)} D_{T}<F F_{j-2} B^{-(j+1)} D_{U}
$$

The unique child of $Q$ is $F F_{\jmath} B^{-(j+1)} D_{T}$. It is enough to show that $F F_{j-2} B^{-(j+1)} D_{U}<F F_{j} B^{-(j+1)} D_{T}$. Since $F_{j}=F_{j-2} F_{j-1}$, it follows that the successor of $F F_{j} B^{-(j+1)} D_{T}$ is $F F_{j-2} B^{-(\jmath+1)} D_{U}$. Since in the generation $j+1$, the succession is opposite to the order, we are done. (Notice that $F F_{j-2} F_{j-1}$ is not of maximal length in $\left\{F_{j+1}\right\}$, because otherwise $F F_{j-2}$ would be of maximal length in $\left\{F_{j}\right\}$.)

Third case: $P=F B^{-j} D_{T}<Q=F F_{j-1} B^{-j} D_{T}$. (This case implies $j>0$.) Here, $F$ is in $\left\{F_{j-2}\right\}$. These two patches give rise to four children with the siblings being neighbors. The progeny of $P$ consists of two patches that are ordered as follows:

$$
F B^{-(j+1)} D_{T}<F B^{-(j+1)} D_{U} \text {. }
$$

The progeny of $Q$ consists of two patches that are ordered as follows:

$$
F F_{j-1} B^{-(j+1)} D_{T}<F F_{j-1} B^{-(j+1)} D_{U} \text {. }
$$

It is enough to show that $F B^{-(j+1)} D_{U}<F F_{j-1} B^{-(j+1)} D_{T}$. This in turn follows from the facts that the successor of $F F_{\jmath-1} B^{-(j+1)} D_{T}$ is $F B^{-(j+1)} D_{U}$ and that the 
generation $j+1$ is even. (Notice that $F F_{j-1}$ is not of maximal length in $\left\{F_{j+1}\right\}$, because it is in $\left\{F_{j}\right\}$.)

Lemma 7.14. Let $C, P, Q$, and $R$ be Fibonacci patches; denote their strings by $F_{C}$, $F_{P}, F_{a}$, and $F_{R}$ respectively; assume that $Q$ and $R$ are tagged by $T$, and that $C$ descends from $P$. Then the following implications hold:

$$
Q<P \Rightarrow F_{Q} \leq F_{C}, \quad P<R \Rightarrow F_{C} \leq F_{R} .
$$

Proof. If $Q$ is tagged by $T$, then the string $F_{Q}$ belongs to the family-heritage; more precisely: in each generation, there is a descendent of $Q$ that carries the string $F_{Q}$. The first claim now follows from Lemma 7.13 by noting that for any two patches $A$ and $B$ the relation $A<B$ implies $F_{A} \leq F_{B}$. The second claim is proved in the same way.

Now we are going to show that only neighboring patches overlap. For this purpose, we have to impose four additional assumptions on the fixed point, namely:

A5.

$$
\begin{aligned}
U \overline{D_{T}} \cap \overline{D_{U}} & =\emptyset . \\
B^{-1} \overline{D_{T}} \cap U B^{-1} \overline{D_{T}} & =\emptyset, \\
B^{-1} \overline{D_{T}} \cap U B^{-1} \overline{D_{U}} & =\emptyset, \\
B^{-1} \overline{D_{U}} \cap U B^{-1} \overline{D_{U}} & =\emptyset .
\end{aligned}
$$

(Remember: $U$ has been continued to $D_{U} \cup D_{T}$, see Sect. 1. In fact, it is continuous up to the closure of this set, by the domain extension property.)

It is possible to find domains such that these hold in addition to our old assumptions, both for an approximate critical fixed point and for the simple fixed point. We call them disjointness assumptions.

Lemma 7.15. Fibonacci patches of the same generation overlap if and only if they are neighbors. If they are not neighbors, then even their closures are disjoint.

Proof. Ther "if" part of the first assertion has already been proved in Lemma 7.10. The "only if" part will follow from the second assertion.

For the zeroth generation, the claim is vacuously true, since $D_{U}$ and $D_{T}$ are neighbors. Let $j$ be greater than or equal to zero and assume that the claim has been proved up to $j$. Assume that the closures of two patches of the generation $j+1$ overlap without the patches being neighbors. Since siblings are neighbors, it follows that the parents of the patches are distinct. Since, by Lemma 7.12, children (including their closure) are contained in their parents, it follows that the parents overlap too. By the inductive assumption, it follows that the parents are neighbors. Let us denote the parents by $P$ and $Q$. We distinguish the same cases as in the proof of Lemma 7.13:

First case: $P=F B^{-j} D_{U}$ and $Q=F B^{-j} D_{T}$. The unique child of $P$ is

$$
F F_{j} B^{-(\jmath+1)} D_{T} \text {. }
$$

The progeny of $Q$ consists of two neighboring patches, namely

$$
F B^{-(j+1)} D_{T} \text { and } F B^{-(j+1)} D_{U} \text {. }
$$

Since $F F_{j} B^{-(j+1)} D_{T}$ and $F B^{-(j+1)} D_{T}$ are neighbors, our assumption implies that

$$
F F_{j} B^{-(j+1)} \overline{D_{T}} \cap F B^{-(j+1)} \overline{D_{U}} \neq \emptyset .
$$


Applying $F^{-1}$ and making use of Lemma 2.2, we end up with the relation

$$
U \overline{D_{T}} \cap \overline{D_{U}} \neq \emptyset \text {, }
$$

which contradicts the first disjointness assumption.

Second case: $P=F F_{j-2} B^{-j} D_{T}$ and $Q=F B^{-j} D_{U}$. The progeny of $P$ consists of two neighboring patches, namely

$$
F F_{j-2} B^{-(j+1)} D_{T} \text { and } F F_{\jmath-2} B^{-(j+1)} D_{U} .
$$

The unique child of $Q$ is $F F_{j} B^{-(\jmath+1)} D_{T}$. Since $F F_{j-2} B^{-(j+1)} D_{U}$ and $F F_{\jmath} B^{-(j+1)} D_{T}$ are neighbors, our assumption implies that

$$
F F_{j-2} B^{-(j+1)} \overline{D_{T}} \cap F F_{j} B^{-(j+1)} \overline{D_{T}} \neq \emptyset .
$$

We have $F_{j}=F_{j-2} F_{j-1}$. Applying $\left(F F_{j-2}\right)^{-1}$ and making use of Lemma 2.2, we end up with the relation

$$
B^{-1} \overline{D_{T}} \cap U B^{-1} \overline{D_{T}} \neq \emptyset
$$

which contradicts the second disjointness assumption.

Third case: $P=F B^{-j} D_{T}$ and $Q=F F_{j-1} B^{-j} D_{T}$. These two patches give rise to four children with the siblings being neighbors. The progeny of $P$ consists of two patches, namely

$$
F B^{-(j+1)} D_{T} \text { and } F B^{-(\jmath+1)} D_{U} \text {. }
$$

The progeny of $Q$ consists of two patches, namely:

$$
F F_{j-1} B^{-(j+1)} D_{T} \text { and } F F_{j-1} B^{-(j+1)} D_{U} .
$$

Since $F B^{-(j+1)} D_{U}$ and $F F_{j-1} B^{-(j+1)} D_{T}$ are neighbors, our assumption leads to three subcases:

First subcase:

$$
F B^{-(\jmath+1)} \overline{D_{T}} \cap F F_{\jmath-1} B^{-(j+1)} \overline{D_{T}} \neq \emptyset .
$$

This leads to the relation

$$
B^{-1} \overline{D_{T}} \cap U B^{-1} \overline{D_{T}} \neq \emptyset,
$$

which contradicts the second disjointness assumption.

Second subcase:

$$
F B^{-(j+1)} \overline{D_{T}} \cap F F_{\jmath-1} B^{-(\jmath+1)} \overline{D_{U}} \neq \emptyset .
$$

This leads to the relation

$$
B^{-1} \overline{D_{T}} \cap U B^{-1} \overline{D_{U}} \neq \emptyset,
$$

which contradicts the third disjointness assumption.

Third subcase:

$$
F B^{-(j+1)} \overline{D_{U}} \cap F F_{j-1} B^{-(\jmath+1)} \overline{D_{U}} \neq \emptyset .
$$

This leads to the relation

$$
B^{-1} \overline{D_{U}} \cap U B^{-1} \overline{D_{U}} \neq \emptyset,
$$

which contradicts the fourth disjointness assumption. 
As in Sect. 1, let $L$ be the limit set of the $L_{j}$. We know that $L$ is non-empty, compact, and connected. We are going to show that $L$ is homeomorphic to an interval. For this purpose, we define a sequence of numerical functions $\psi_{j}$ as follows:

Definition 7.16. Let $z$ be a point of $L_{j}$ with $j \geq 0$. Then, $z$ is contained in the closure of at least one Fibonacci patch of the $j^{\text {th }}$ generation. (In fact, it is contained in the interior of at least one patch.) Let $F$ be the corresponding Fibonacci tail. In case of a tie, i.e., if $z$ lies in the closure of exactly two Fibonacci patches with different strings, we choose the left string, more precisely: the one for which $\varrho(z)$ is minimal. Then, we put

$$
\psi_{j}(z)=\varrho(F) .
$$

(Notice that by Lemma 7.15, a point cannot be contained in the closure of more than two patches.)

Lemma 7.17. $\lim _{\jmath \rightarrow \infty} \psi_{\jmath}(z)$ exists for each $z \in L$.

Proof. For two neighboring patches $P$ and $Q$ of the same generation $j \geq 1$, with corresponding Fibonacci tails $F_{P}$ and $F_{Q}$ respectively, we have $\left|\varrho\left(F_{P}\right)-\varrho\left(F_{Q}\right)\right| \leq$ $\left|\varrho\left(F_{j-2}\right)\right|$ by Definition 7.8. By Lemma 7.6, the latter number is equal to $\omega^{j-1}$.

Take any $z \in L$ and fix $j \geq 1$. In particular, we have $z \in L_{\jmath}$. Denote by $P$ the smallest (leftmost) patch of the $j^{\text {th }}$ generation the closure of which contains $z$. If there is a patch larger than $P$, tagged by $T$, and such that its closure does not contain $z$, we denote the smallest one of these by $R$; otherwise let $R$ be the right boundary patch. In the same way: if there is a patch smaller than $P$, tagged by $T$, and such that its closure does not contain $z$, we denote the largest of these by $Q$; otherwise let $Q$ be the left boundary patch. Then we have

$$
Q \leq P \leq R
$$

We call the patches $Q$ and $R$ bracketing patches for the point $z$. (For later use, we note that $Q$ and $R$ are bracketing patches for all points in a small open neighborhood of $z$ as well.) Because a point cannot belong to the closure of more than two patches (see Lemma 7.15), and because patches tagged by $U$ cannot be neighbors (see Definition 7.8), it follows that there are not more than four patches of the $j^{\text {th }}$ generation strictly between $Q$ and $R$. By the above remark, this gives the estimate

$$
\left|\varrho\left(F_{Q}\right)-\varrho\left(F_{R}\right)\right| \leq 6 \omega^{j-1} .
$$

For each $k>j$, denote by $P_{k}$ the smallest patch of the $k^{\text {th }}$ generation the closure of which contains $z$.

Assume first that neither $Q$ nor $R$ are boundary patches. Then, by definition, $z$ is contained neither in the closure of $Q$ nor in the closure of $R$. Moreover, it follows from Lemma 7.15 that $z$ is not contained in the closure of any patch (of generation $j$ ) that is either smaller than $Q$ or greater than $R$. Since by Lemma 7.12, patches are contained in their ancestors, none of the $P_{k}$ can descend from a patch (of the $j^{\text {th }}$ generation) less than or equal to $Q$, or from a patch (of the $j^{\text {th }}$ generation) greater than or equal to $R$. It follows that each $P_{k}$ descends from a patch strictly between $Q$ and $R$. Since $Q$ and $R$ are tagged by $T$, Lemma 7.14 yields

$$
F_{Q} \leq F_{P_{k}} \leq F_{R}, \quad \text { for } k>j
$$


and it follows that

$$
\varrho\left(F_{Q}\right) \leq \psi_{k}(z) \leq \varrho\left(F_{R}\right), \quad \text { for } \quad k>j .
$$

Assume now that $R$ is the right boundary patch. In this case, we have the inequality

$$
\varrho\left(F_{P_{k}}\right) \leq 1, \quad \text { for } k>j,
$$

since the $\varrho$-values of the right boundary strings converge to 1 from below by Lemma 7.7 .

In the same way, if $Q$ is the left boundary patch, then we have the inequality

$$
-\omega \leq \varrho\left(F_{P_{k}}\right), \text { for } k>j,
$$

since the $\varrho$-values of the left boundary strings converge to $-\omega$ from above.

In any case, we end up with an interval $[a, b]$ such that $a \leq \psi_{k}(z) \leq b$ for $k>j$. This interval is called a bracketing interval for the point $z$. (For later use, note that the interval $[a, b]$ is a bracketing interval for the points in a small open neighborhood of $z$ as well.) It follows that the numbers $\psi^{-}(z)=\liminf _{k \rightarrow \infty} \psi_{k}(z)$ and $\psi^{+}(z)=\limsup _{k \rightarrow \infty} \psi_{k}(z)$ are both contained in the bracketing interval. By the above estimate, the size of the bracketing interval is bounded by $6 \omega^{j-1}$. Since $j$ was arbitrary, the numbers $\psi^{-}(z)$ and $\psi^{+}(z)$ coincide, and the claim follows.

Using the preceding lemma, we define

$$
\psi(z)=\lim _{\jmath \rightarrow \infty} \psi_{\jmath}(z)
$$

for $z \in L$.

Lemma 7.18. The function $\psi$ is continuous.

Proof. Fix $z$ in $L$. For an arbitrary $j$, we construct the bracketing patches and the bracketing interval as in the proof of Lemma 7.17.

If has already been observed that there is a small open neighborhood of $z$, such that for each point of this neighborhood, the same bracketing patches are valid. Therefore, the range of $\psi$ on this small open set is contained in the bracketing interval; this one can be made arbitrarily small by choosing $j$ large enough.

Lemma 7.19.

$$
\psi(L)=[-\omega, 1] .
$$

Proof. Lemma 7.18 implies that $\psi(L)$ is a compact connected subset of the real axis, i.e., a closed bounded interval. It follows from Lemma 7.7 that this interval is equal to $[-\omega, 1]$.

Definition 7.20. Fix a non-negative integer $j$. Let $z$ and $w$ be elements of the set $L_{j}$. Let $P$ and $Q$ be the smallest patches of the $j^{\text {th }}$ generation the closure of which contains $z$ and $w$ respectively. Without loss of generality, we assume that $P \leq Q$. We say that $z$ and $w$ are separated by $m$ patches of the $j^{\text {th }}$ generation if there are $m$ patches $R_{1}, R_{2}, \ldots, R_{m}$ of the $j^{\text {th }}$ generation such that

$$
P<R_{1}<R_{2}<\ldots<R_{m}<Q .
$$


Lemma 7.21. Let $z$ and $w$ be contained in the set $L$ and assume that there exists a non-negative integer $j$ such that

$$
|\psi(z)-\psi(w)|<\omega^{j}
$$

Then $z$ and $w$ are not separated by more than seven patches of the $j^{\text {th }}$ generation.

Proof. We prove that $z$ and $w$ cannot be separated by more than three patches of the $j^{\text {th }}$ generation that are tagged by $T$. The claim then follows by observing that patches tagged by $U$ cannot be neighbors (see Definition 7.11).

Assume to the contrary that $z$ and $w$ are separated by more than three patches of the $j^{\text {th }}$ generation that are tagged by $T$. Then there exist patches $R_{1}, \ldots, R_{4}$ of the $j^{\text {th }}$ generation, tagged by $T$, such that

$$
P<R_{1}<R_{2}<R_{3}<R_{4}<Q,
$$

and (without loss of generality) $z \in \bar{P}$ and $w \in \bar{Q}$. For all $k>j$, let $P_{k}$ and $Q_{k}$ be the smallest patches of the $k^{\text {th }}$ generation the closure of which contains $z$ and $w$ respectively. By Lemma 7.15, $z$ does not belong to the closure of $R_{2}$, nor to the closure of any patch (of generation $j$ ) that is greater than $R_{2}$. In the same way, $w$ does not belong to the closure of $R_{3}$, nor to the closure of any patch (of generation $j$ ) that is smaller than $R_{3}$. Since patches are contained in their ancestors (see Lemma 7.15), it follows that the $P_{k}$ descend from patches (of the $j^{\text {th }}$ generation) that are strictly smaller than $R_{2}$, and the $Q_{k}$ from patches (of the $j^{\text {th }}$ generation) that are strictly greater than $R_{3}$.

Let $F$ and $G$ denote the strings corresponding to $R_{2}$ and to $R_{3}$ respectively. Since $R_{2}$ and $R_{3}$ have the same tag, $F$ is strictly smaller than $G$. Let $F_{k}$ and $G_{k}$ denote the strings corresponding to $P_{k}$ and $Q_{k}$ respectively. Since $R_{2}$ and $R_{3}$ are tagged by $T$, it follows from Lemma 7.14 that we have

$$
F_{k} \leq F<G \leq G_{k}, \text { for } k>j .
$$

This implies

$$
\psi_{k}(z) \leq \varrho(F)<\varrho(G) \leq \psi_{k}(w), \quad \text { for } \quad k>j .
$$

By passing to the limit, we obtain

$$
\psi(z) \leq \varrho(F)<\varrho(G) \leq \psi(w) .
$$

Since $R_{2}$ and $R_{3}$ are tagged by $T$, it follows from Definition 7.8 (and from the monotonicity of $\varrho$ ) that $|\varrho(F)-\varrho(G)| \geq\left|\varrho\left(F_{j-1}\right)\right|$, and by Lemma 7.6, the latter number is equal to $\omega^{\jmath}$. It follows that $|\psi(z)-\psi(w)| \geq \omega^{j}$, and we have reached the desired contradiction.

Lemma 7.22. The function $\psi$ is one-to-one.

Proof. The claim follows from Lemma 7.21 by observing that the maximal diameter of the patches of the $j^{\text {th }}$ generation converges to zero with increasing $j$.

Lemma 7.23. The function $\psi$ is a homeomorphism. The set $L$ is a continuous curve.

Proof. The first claim follows from Lemma 7.18 and Lemma 7.22 by general topology, by observing that $\mathbb{R}$ is a Hausdorff space.

It now follows from Lemma 7.19 that the set $L$ is homeomorphic to a closed bounded interval, i.e., that it is a continuous curve. 
Recall from Sect. 2 the definition of the next symbol: the next symbol $\nu(F)$ of a Fibonacci tail $F$ is the first symbol of the Fibonacci tail of minimal length that strictly contains $F$. Let $P$ be a Fibonacci patch and denote its string by $F$. Now, we define the next symbol of the patch $P$ by $\nu(P)=\nu(F)$. Guided by Lemma 2.9, we define the sets $M_{j}$ and $N_{\jmath}$ as follows:

Definition 7.24. For any $j \geq 1$, the set $M_{j}$ (respectively $N_{j}$ ) is defined to be the union of the patches of generation $j$ the next symbol of which is equal to $U$ (respectively $T)$.

(We have to exclude the case $j=0$, since $M_{0}=D_{U}$, but $\nu\left(D_{U}\right)=T$ according to our definition. Alternatively, we could simply define the next symbol of the patch $D_{U}$ to be equal to $U$, arguing that for the empty string in $\left\{F_{-1}\right\}$, the next symbol is $U$.)

Lemma 7.25. Assume that $F \in\left\{F_{j-1}\right\}$ with $j \geq 1$. Then we have

$$
\nu\left(F F_{j}\right)=\nu(F) .
$$

Proof. According to the assumption, there exists a string $\Delta$ different from the empty string such that $\Delta F=F_{\jmath_{-1}}$; in particular, $\Delta F$ is a Fibonacci tail.

Because of the equation $F_{j+1}=F_{j-1} F_{\jmath}$, we have $\Delta F F_{j}=F_{\jmath+1}$; in particular, $\triangle F F_{j}$ is a Fibonacci tail.

We have shown that both $\Delta F$ and $\Delta F F_{j}$ are Fibonacci tails. The claim now follows by observing that $\Delta$ is not empty.

Lemma 7.26. Let $P$ be any patch with generation $j \geq 2$. Then we have

$$
\nu(P)=\nu(\pi(P)) .
$$

Lemma 7.26 says that the next symbol is inherited.

Proof. Looking at Definition 7.11, we see that we only have to prove that

$$
\nu(F)=\nu\left(F-F_{j}\right) \quad \text { for } \quad F \in\left\{F_{j-1}\right\} F_{j} \quad \text { and } \quad j \geq 1 .
$$

But this is just Lemma 7.25 in a different disguise.

Lemma 7.27. Let $P$ be any patch with generation $j \geq 2$ that has a successor, and let $F$ be its string. Then we have $\nu(P)=\nu(\sigma(P))$, unless $P$ is tagged by $T$ and

$$
F_{j}= \begin{cases}T U F, & \text { for even } j \\ U T F, & \text { otherwise. }\end{cases}
$$

Lemma 7.27 says that the next symbol jumps exactly once in a fixed generation. The above patch, at which the jump occurs, will be called the pivotal patch. (Incidentally, the string of the pivotal patch is palindromical. It is easy to prove this by induction.)

Proof. According to Definition 7.8, the next symbol can change only in two cases, namely

- between patches of the form $F B^{-j} D_{T}$ and $F F_{\jmath-1} B^{-\jmath} D_{T}$, and

- between patches of the form $F B^{-\jmath} D_{T}$ and $\left(F-F_{j-2}\right) B^{-j} D_{U}$.

Lemma 7.25 excludes a change in the first case. (We have $F \in\left\{F_{\jmath_{-2}}\right\}$.) It remains to discuss the second one.

Assume, therefore, that $F \in\left\{F_{j}\right\} \backslash\left\{F_{j-2}\right\}$ and that $F \neq \hat{F}_{j}$. 
We only discuss the case of $j$ being even. (For odd values of $j$, the argument is completely analogous.) Since for even values of $j$ (with $j \geq 2$ ), the Fibonacci string $F_{\text {j }}$ starts with $T U$ (see Lemma 7.5), we can write

$$
F_{j}=T U \Delta F,
$$

with a string $\Delta$ that is possibly empty. By definition, $\Delta F$ is a Fibonacci tail.

By assumption, the string $F$ is strictly longer than $F_{j-2}$. Therefore, we have

$$
F_{j}=T U \Delta G F_{j-2},
$$

and by Lemma 7.5 , the string $\Delta G$ is a Fibonacci tail. (Indeed, $\Delta G \in\left\{F_{j-1}\right\}$.)

Therefore, both $\Delta F$ and $\Delta G$ are Fibonacci tails. If $\Delta$ is not the empty string, it follows that $\nu(F)=\nu(G)=\nu\left(F-F_{j-2}\right)$.

The only case, therefore, where a jump is not excluded, is the case of $\Delta$ being the empty string. Since the next symbols of $\hat{F}_{\jmath}$ and $\hat{F}_{\jmath-1}$ are always different (see Lemma 7.5), it follows that a jump must occur. We conclude that it occurs exactly for $\Delta$ being the empty string, and this was to be shown.

Let us collect a few consequences of what we have proved so far. It follows from the preceding lemma and from Lemma 7.10 that the sets $M_{\jmath}$ and $N_{j}$ are connected and that they overlap. Moreover, the sequences $\left(M_{j}\right)$ and $\left(N_{j}\right)$ decrease - this follows from Lemma 7.12, by observing that the next symbol is inherited (see Lemma 7.26). As in Sect. 1, we conclude that the limit sets $M$ and $N$ are non-empty, compact, and connected, and that we have $L=M \cup N$. Furthermore, the sets $M$ and $N$ intersect. Since the maximal diameter of the patches converges to zero with increasing generation, $M$ and $N$ intersect in exactly one point.

Again, since $\psi$ is continuous (see Lemma 7.18), it follows that $\psi(M)$ and $\psi(N)$ are closed bounded intervals. Since $\psi$ is injective (see Lemma 7.22) and since $M$ and $N$ intersect in exactly one point, the same is true for these intervals.

By the preceding lemma, the string $F_{P}$ of the pivotal patch is obtained by removing the symbols $U T$ or $T U$ from the head of the string $F_{j}$. Since $\lim _{j \rightarrow \infty} \varrho\left(F_{j}\right)=0$ by Lemma 7.6, it follows that $\varrho\left(F_{P}\right)$ converges to $1-\omega$ with increasing generation. We conclude that both $\psi(M)$ and $\psi(N)$ contain the point $1-\omega$. Since for even values of $j$, the next symbol of $\hat{F}_{\jmath}$ is equal to $T$ (see Lemma 7.5), it follows from Lemma 7.7 that $-\omega$ is contained in $\psi(N)$. Summing up, we have proved the following result:

Lemma 7.28.

$$
\psi(N)=[-\omega, 1-\omega], \quad \psi(M)=[1-\omega, 1] .
$$

We have $\psi(0,0)=0$, since $(0,0)$ is contained in all the patches of the form $B^{-j} D_{T}$.

\section{Lemma 7.29.}

$$
\begin{aligned}
& \psi(U z)=\psi(z)-1 \quad \text { for } \quad z \in M \\
& \psi(T z)=\psi(z)+\omega \quad \text { for } \quad z \in N .
\end{aligned}
$$

Proof. Take any $z \in L$ that is not a boundary point. It follows that for sufficiently high values of $j$, the point $z$ is not contained in the closure of a boundary patch of generation $j$.

Assume that $z \in M$. In particular, we have $z \in M_{j}$. Since $z$ is not contained in the closure of a boundary patch, we have $U z \in M_{\jmath}$, and it follows that

$$
\psi_{j}(U z)=\psi_{j}(z)-1,
$$

since adding a symbol $U$ to a string changes its $\varrho$-value by -1 . 
Since $j$ was arbitrary, it follows that $\psi(U z)=\psi(z)-1$. If $z$ is a boundary point, the claim follows by continuity.

The second claim is proved in the same way.

We denote the inverse of $\psi$ by $\phi$.

Lemma 7.30. We have

$$
\begin{gathered}
\phi(t-1)=U \phi(t) \quad \text { for } \quad 1-\omega \leq t \leq 1, \\
\phi(t+\omega)=T \phi(t) \quad \text { for } \quad-\omega \leq t \leq 1-\omega,
\end{gathered}
$$

which means that $\phi$ conjugates the induced pair to a rigid rotation.

Proof. The claim immediately follows from Lemma 7.28 and Lemma 7.29.

The pair $([1-\omega, 1],[-\omega, 1-\omega])$ is invariant, in the sense of Definition 3.1, for the pair of maps $(u, t)$ defined by

$$
u(s)=s-1, \quad t(s)=s+\omega .
$$

Since $\phi$ is a homeomorphism, we conclude that $(M, N)$ is invariant for the pair $(U, T)$.

Lemma 7.31. The curve $t \rightarrow \phi(t)$ is Hölder continuous.

Proof. By assumption A4, there is a number $c$ with $0<c<1$ such that

$$
\left|T B^{-2} z-T B^{-2} w\right| \leq c|z-w|
$$

for arbitrary $z$ and $w$ in $D_{U} \cup D_{T}$.

We put

$$
\kappa=\max \left\{\frac{1}{|\alpha|}, \frac{1}{|\beta|}, c\right\},
$$

where $\alpha$ and $\beta$ are the diagonal elements of the rescaling $B$. By assumption, we have $0<\kappa<1$. It follows from Lemma 1.5 that the maximal diameter of the patches of the $j^{\text {th }}$ generation is bounded by $d \kappa^{j}$ with a positive constant $d$.

Finally, we put

$$
\mu=\frac{\log \kappa}{\log \omega} .
$$

The positive number $\mu$ is going to be the Hölder exponent.

We fix $j \geq 1$ and choose any two different points $s$ and $t$ in the interval $\psi(L)$ with $\omega^{j+1} \leq|s-t|<\omega^{j}$.

It follows from Lemma 7.21 that the points $\phi(s)$ and $\phi(t)$ cannot be separated by more than seven patches of the $j^{\text {th }}$ generation, and we obtain the estimate

$$
|\phi(s)-\phi(t)| \leq 9 d \kappa^{\jmath} .
$$

It follows that

$$
\frac{|\phi(s)-\phi(t)|}{|s-t|^{\mu}} \leq \frac{9 d}{\omega^{\mu}}\left(\frac{\kappa}{\omega^{\mu}}\right)^{j}=\frac{9 d}{\omega^{\mu}} .
$$

Since $j$ was arbitrary, we are done.

In the case of the simple fixed point, where $B=\operatorname{diag}\left(-1 / \omega,-1 / \omega^{2}\right)$, and where $c=\omega^{2}$, the number $\mu$ becomes equal to one, and it follows that the curve $t \mapsto \phi(t)$ is Lipschitz continuous. In the case of the critical fixed point, the number $\mu$ is strictly smaller than one. (We have $0.618 \approx \omega<1 /|\alpha| \approx 0.71$ and $1 /|\alpha| \leq \kappa<1$.) 
Lemma 7.32. Under the conditions A1-A5, the number

$$
\mu=\frac{\log |\alpha|}{\log \frac{1}{\omega}}
$$

is an upper bound for the Hölder exponent of the conjugator at the origin. Here, $\alpha$ is the smaller (in absolute value) of the diagonal elements of the rescaling $B$.

Proof. For arbitrary $j$, let the $j^{\text {th }}$ Fibonacci string $F_{j}$ be characterized by the pair $\left(p_{\jmath}, q_{j}\right)$. It follows from Lemma 7.30 that

$$
\phi\left(t+q_{j} \omega-p_{j}\right)=F_{\jmath} \phi(t) .
$$

In particular, this holds for $t=0$, and by making use of Lemma 2.2, we obtain

$$
\phi\left(q_{j} \omega-p_{j}\right)=F_{j}(0,0)=B^{-j} T(0,0)=\left(\begin{array}{c}
\frac{c_{1}}{\alpha^{j}} \\
\frac{c_{2}}{\beta^{\jmath}}
\end{array}\right),
$$

where $c_{1}$ and $c_{2}$ are the coordinates of the point $T(0,0)$ and where $\alpha$ and $\beta$ are the diagonal elements of the rescaling $B$. We still assume that $\alpha$ is the smaller one in absolute value.

For the next step, we need $c_{1}$ to be different from zero. Of course, we could simply assume this and justify the assumption by numerics. But there is a simple a priori argument proving it: we put $z=(0,0)$ and $w=T z$ and assume that the first coordinate of $w$ is equal to zero. Then we have $S z=z$ and $S w=w$; the equation $z=T^{-1} w$ now implies $S z=T^{-1} S w$, and by symmetry, it follows that $z=T w$. Combining this equation with $z=T^{-1} w$, it follows that $z=T^{-2} z$, i.e., that $z$ is a fixed point of the map $T^{2}$, and this contradicts the fact that the rotation number of $z$ is well defined and equal to $\omega$. (The latter claim is a consequence of Lemma 7.30.)

Assume that the curve is Hölder continuous at the origin with an exponent $0<\nu \leq 1$. There exists a positive constant $c$ such that

$$
\left|\phi\left(q_{j} \omega-p_{j}\right)\right| \leq c\left|q_{\jmath} \omega-p_{\jmath}\right|^{\nu} \leq c \omega \omega^{\jmath \nu},
$$

where we have used Lemma 7.6. On the other hand, we obtain

$$
\left|\phi\left(q_{j} \omega-p_{\jmath}\right)\right|=\left|\left(\begin{array}{c}
\frac{c_{1}}{\alpha^{\jmath}} \\
\frac{c_{2}}{\beta^{\jmath}}
\end{array}\right)\right| \geq\left|c_{1}\right||\alpha|^{-j} .
$$

Combining the last two inequalities gives

$$
\left|c_{1}\right||\alpha|^{-j} \leq c \omega \omega^{\jmath \nu}
$$

Since $j$ was arbitrary, it follows that $\omega^{\nu} \geq|\alpha|^{-1}$. (Remember that $c_{1}$ is different from zero.) This, in turn, implies

$$
\nu \leq-\frac{\log |\alpha|}{\log \omega}=\mu
$$

and we are down.

For maps attracted by the critical fixed point, the numerical value of $\mu$ is 0.721 . In particular, the conjugator of the critical golden curve is not differentiable at the origin. (It follows that it is not differentiable on the orbit of the origin, which is dense.) 
We are going to show that the Hölder continuity holds with the above number $\mu$ as exponent; it will follow that $\mu$ is the optimal Hölder exponent. For this purpose, let us impose an additional assumption on the fixed point, namely:

A6. The map $T B^{-1}$ is a uniform contraction on $D_{U} \cup D_{T}$.

(A direct calculation shows that this condition is satisfied for the simple fixed point. In the case of the critical fixed point, it looks as if it was satisfied too, but the numerical evidence is not conclusive.)

Lemma 7.33. Under the conditions A1-A6, the Hölder continuity holds with the number $\mu$ from Lemma 7.32 as exponent.

Proof. Since $T B^{-1}$ is a contraction, the number $c$ in the proof of Lemma 7.31 becomes smaller than $1 /|\alpha|$, and it follows that we have $\kappa=1 /|\alpha|$. The claim now follows from the proof of Lemma 7.31.

By Lemma 7.32, the Hölder exponent given by Lemma 7.33 is optimal.

In the appendix, we prove that all these results hold for asymptotic self-similarity as well. Applying the construction of Sect. 5 to the parametrization $t \mapsto \phi(t)$ yields a Hölder continuous curve going around the cylinder; the induced mapping on the curve is conjugate to a rigid rotation. Thus we obtain the following result:

Theorem 7.34. If a homeomorphism of the cylinder is attracted by a fixed point that satisfies the assumptions A1-A5, then it has an invariant golden circle, and the induced mapping on the curve is continuously conjugate to a rigid rotation. In particular, the invariant curve is topologically transitive. Moreover, the conjugator is Hölder continuous. For maps attracted by the critical fixed point, the conjugator is not differentiable on a dense set of points.

\section{Appendix}

For the sake of completeness, we give the proofs showing that the theory of Sect. 7 carries over to the case of asymptotic self-similarity. Moreover, we repeat the procedure of Sect. 5 in order to obtain a parametrized necklace set.

The combinatorial part of Sect. 7 does not require any changes. We only have to worry about the results that involve topological properties of the patches, namely Lemma 7.10, Lemma 7.12, and Lemma 7.15.

(The proof of Lemma 7.31 requires a small change that we leave out. Furthermore, in the proof of Lemma 7.33, the powers $\alpha^{-j}$ and $\beta^{-j}$ must be replaced by $\alpha_{1}^{-1} \alpha_{2}^{-1} \ldots \alpha_{j}^{-1}$ and by $\beta_{1}^{-1} \beta_{2}^{-1} \ldots \beta_{j}^{-1}$ respectively; apart from this modification, the proof stays valid.)

Let us recall the terminology from Sect. 4. The asymptotically self-similar pair is denoted by $\left(U_{0}, T_{0}\right)$. We consider the converging sequence

$$
\left(U_{k+1}, T_{k+1}\right)=B_{k+1}\left(T_{k}, U_{k} T_{k}\right) B_{k+1}^{-1}, \quad k \geq 0
$$

of iterated images of $\left(U_{0}, T_{0}\right)$ under the renormalization operator. The index $k$ denotes the iteration step number along the orbit; it will keep this meaning in the whole section.

The following conditions are assumed to hold for all non-negative integers $k$ :

$$
B_{k+1}^{-1} \overline{D_{U}} \subset D_{T}, \quad B_{k+1}^{-1} \overline{D_{T}} \subset D_{T}, \quad T_{k} B_{k+1}^{-1} \overline{D_{T}} \subset D_{U} .
$$


(These follow from the domain extension property and from convergence to the fixed point.)

$$
D_{U} \cap D_{T} \neq \emptyset, \quad U_{k} D_{T} \cap D_{U} \neq \emptyset, \quad U_{k} B_{k+1}^{-1} D_{T} \cap B_{k+1}^{-1} D_{U} \neq \emptyset .
$$

(These follow from the connectedness assumptions and from convergence to the fixed point.)

Recall from Lemma 4.10 the following representation of the sets $L_{j}^{k}$ :

$$
L_{j}^{k}=\left\{F_{j}^{k}\right\} B_{k+1}^{-\jmath} D_{T} \cup\left\{F_{j-1}^{k}\right\} B_{k+1}^{-j} D_{U}
$$

for pairs $(j, k)$ of non-negative integers.

Now, the patches obtain a second index $k$ :

Definition 8.1. A patch descriptor is an object consisting of a non-negative integer $j$, denoting what we call its generation, a non-negative integer $k$, selecting the pair $\left(U_{k}, T_{k}\right)$ to be used, a Fibonacci tail $F$, and a tag that is either the symbol $U$ or the symbol T. $F$ is required to be a member of $\left\{F_{j-1}\right\}$ if the tag is equal to $U$, and a member of $\left\{F_{j}\right\}$ otherwise.

Lemma 4.10 shows that the patch descriptors are in a one-to-one correspondence with the patches.

Descendence and succession are now defined to relate only patches with the same value of $k$. Apart from this, they are defined exactly as before. At last, we shall only need the patches with $k=0$, the set $L^{0}$ being invariant for the initial pair $\left(U_{0}, T_{0}\right)$. But the intermediate steps involve all values of $k$. In particular, the domain extension property and the connectedness assumptions will be used with higher and higher values of $k$ as we descend in the hierarchy of the patches.

Lemma 8.2. If two patches of the same generation are neighbors, then they overlap as sets.

Proof. The condition of being neighbors implies that the patches have the same index $k$.

According to Definition 7.8, there are three possible cases, each of which is reduced to one of the connectedness assumptions. Apart from Lemma 4.8, the reductions use the fact that the maps $U_{l}, T_{l}$, and $B_{l+1}$ are homeomorphisms.

Recall Lemma 4.8:

$$
\begin{gathered}
T_{k+j}=F_{0}^{k+\jmath}=B_{k+1}^{\jmath} F_{\jmath}^{k} B_{k+1}^{-j}, \quad j \geq 0, \\
U_{k+j+1}=F_{-1}^{k+\jmath+1}=B_{k+1}^{\jmath+1} F_{\jmath}^{k} B_{k+1}^{-(j+1)}, \quad j \geq 0,
\end{gathered}
$$

where we again use the shorthand notations

$$
B_{k+1}^{-j}=B_{k+1}^{-1} B_{k+2}^{-1} \ldots B_{k+j}^{-1}, \quad B_{k+1}^{j}=B_{k+j} B_{k+j-1} \ldots B_{k+1} .
$$

In the following, any string with a raised index $l \geq 0$ denotes a string involving the symbols $U_{l}$ and $T_{l}$.

First case:

$$
F^{k} B_{k+1}^{-j} D_{U} \cap F^{k} B_{k+1}^{-j} D_{T} \neq \emptyset \Leftrightarrow D_{U} \cap D_{T} \neq \emptyset .
$$

Second case:

$$
\begin{aligned}
F^{k} B_{k+1}^{-j} D_{T} \cap F^{k} F_{j-1}^{k} B_{k+1}^{-j} D_{T} \neq \emptyset & \Leftrightarrow B_{k+1}^{-j} D_{T} \cap F_{j-1}^{k} B_{k+1}^{-j} D_{T} \neq \emptyset \\
& \Leftrightarrow D_{T} \cap U_{k+j} D_{T} \neq \emptyset .
\end{aligned}
$$


(Remember: the maps $U_{l}$ have been continued to $D_{U} \cup D_{T}$, see Sect.4.)

Third case:

$$
\begin{aligned}
F^{k} F_{j-2}^{k} B_{k+1}^{-j} D_{T} \cap F^{k} B_{k+1}^{-j} D_{U} \neq \emptyset & \Leftrightarrow F_{j-2}^{k} B_{k+1}^{-\jmath} D_{T} \cap B_{k+1}^{-j} D_{U} \neq \emptyset \\
& \Leftrightarrow U_{k+j-1} B_{k+j}^{-1} D_{T} \cap B_{k+\jmath}^{-1} D_{U} \neq \emptyset .
\end{aligned}
$$

Lemma 8.3. The closure of each patch with $j \geq 1$ is contained in its parent.

Proof. By definition, a child has the same index $k$ as its parent.

According to Definition 7.11, there are three cases to distinguish, each of which is reduced to the domain extension property. Apart from Lemma 4.8, the reductions use the fact that the maps $U_{l}, T_{l}$, and $B_{l+1}$ are homeomorphisms.

As above, any string with a raised index $l \geq 0$ denotes a string involving the symbols $U_{l}$ and $T_{l}$.

First case:

$$
F^{k} B_{k+1}^{-(\jmath+1)} \overline{D_{U}} \subset F^{k} B_{k+1}^{-\jmath} D_{T} \Leftrightarrow B_{k+j+1}^{-1} \overline{D_{U}} \subset D_{T} .
$$

Second case:

$$
F^{k} B_{k+1}^{-(\jmath+1)} \overline{D_{T}} \subset F^{k} B_{k+1}^{-j} D_{T} \Leftrightarrow B_{k+j+1}^{-1} \overline{D_{T}} \subset D_{T} .
$$

Third case:

$$
F^{k} F_{\jmath}^{k} B_{k+1}^{-(\jmath+1)} \overline{D_{T}} \subset F^{k} B_{k+1}^{-j} D_{U} \Leftrightarrow T_{k+j} B_{k+j+1}^{-1} \overline{D_{T}} \subset D_{U} .
$$

Now, we are going to show that only neighboring patches overlap. From the disjointness assumptions (see Sect.7) and from convergence to the fixed point, it follows that the following relations hold for all $k$ greater than a certain $k_{0}$, which we take to be equal to zero without loss of generality.

$$
\begin{aligned}
U_{k} \overline{D_{T}} \cap \overline{D_{U}} & =\emptyset, \\
B_{k+1}^{-1} \overline{D_{T}} \cap U_{k} B_{k+1}^{-1} \overline{D_{T}} & =\emptyset, \\
B_{k+1}^{-1} \overline{D_{T}} \cap U_{k} B_{k+1}^{-1} \overline{D_{U}} & =\emptyset, \\
B_{k+1}^{-1} \overline{D_{U}} \cap U_{k} B_{k+1}^{-1} \overline{D_{U}} & =\emptyset .
\end{aligned}
$$

(Remember: the maps $U_{l}$ have been continued to $D_{U} \cup D_{T}$, see Sect. 4. In fact, they are continuous up to the closure of this set.)

Lemma 8.4. Fibonacci patches of the same generation overlap if and only if they are neighbors. If they are not neighbors, then even their closures are disjoint.

Proof. The "if" part of the first assertion has already been proved in Lemma 8.2. The "only if" part will follow from the second assertion.

For the zeroth generation, the claim is vacuously true, since $D_{U}$ and $D_{T}$ are neighbors. Let $j$ be greater than or equal to zero and assume that the claim has been proved up to $j$. Assume that the closures of two patches of the generation $j+1$ overlap without the patches being neighbors. Since siblings are neighbors, it follows that the parents of the patches are distinct. Since, by Lemma 8.3, children (including their closure) are contained in their parents, it follows that the parents overlap too. By the inductive assumption, it follows that the parents are neighbors. Let us denote the parents by $P$ and $Q$.

As above, any string with a raised index $l \geq 0$ denotes a string involving the symbols $U_{l}$ and $T_{l}$. 
We distinguish the same cases as in the proof of Lemma 7.13:

First case: $P=F^{k} B_{k+1}^{-j} D_{U}$ and $Q=F^{k} B_{k+1}^{-j} D_{T}$. The unique child of $P$ is

$$
F^{k} F_{j}^{k} B_{k+1}^{-(j+1)} D_{T}
$$

The progeny of $Q$ consists of two neighboring patches, namely

$$
F^{k} B_{k+1}^{-(j+1)} D_{T} \quad \text { and } \quad F^{k} B_{k+1}^{-(j+1)} D_{U}
$$

Since $F^{k} F_{j}^{k} B_{k+1}^{-(j+1)} D_{T}$ and $F^{k} B_{k+1}^{-(j+1)} D_{T}$ are neighbors, our assumption implies that

$$
F^{k} F_{j}^{k} B_{k+1}^{-(j+1)} \overline{D_{T}} \cap F^{k} B_{k+1}^{-(j+1)} \overline{D_{U}} \neq \emptyset .
$$

Applying $\left(F^{k}\right)^{-1}$ and making use of Lemma 4.8, we end up with the relation

$$
U_{k+j+1} \overline{D_{T}} \cap \overline{D_{U}} \neq \emptyset,
$$

which contradicts the first disjointness assumption.

Second case: $P=F^{k} F_{j-2}^{k} B_{k+1}^{-\jmath} D_{T}$ and $Q=F^{k} B_{k+1}^{-j} D_{U}$. The progeny of $P$ consists of two neighboring patches, namely

$$
F^{k} F_{j-2}^{k} B_{k+1}^{-(j+1)} D_{T} \text { and } F^{k} F_{\jmath-2}^{k} B_{k+1}^{-(j+1)} D_{U}
$$

The unique child of $Q$ is $F^{k} F_{j}^{k} B_{k+1}^{-(j+1)} D_{T}$. Since the patches $F^{k} F_{j-2}^{k} B_{k+1}^{-(j+1)} D_{U}$ and $F^{k} F_{j}^{k} B_{k+1}^{-(j+1)} D_{T}$ are neighbors, our assumption implies that

$$
F^{k} F_{j-2}^{k} B_{k+1}^{-(j+1)} \overline{D_{T}} \cap F^{k} F_{j}^{k} B_{k+1}^{-(\jmath+1)} \overline{D_{T}} \neq \emptyset .
$$

We have $F_{j}^{k}=F_{j-2}^{k} F_{j-1}^{k}$. Applying $\left(F^{k} F_{j-2}^{k}\right)^{-1}$ and making use of Lemma 4.8, we end up with the relation

$$
B_{k+\jmath+1}^{-1} \overline{D_{T}} \cap U_{k+j} B_{k+\jmath+1}^{-1} \overline{D_{T}} \neq \emptyset,
$$

which contradicts the second disjointness assumption.

Third case: $P=F^{k} B_{k+1}^{-j} D_{T}$ and $Q=F^{k} F_{j-1}^{k} B_{k+1}^{-j} D_{T}$. These two patches give rise to four children with the siblings being neighbors. The progeny of $P$ consists of two patches, namely

$$
F^{k} B_{k+1}^{-(j+1)} D_{T} \quad \text { and } \quad F^{k} B_{k+1}^{-(j+1)} D_{U}
$$

The progeny of $Q$ consists of two patches, namely:

$$
F^{k} F_{j-1}^{k} B_{k+1}^{-(j+1)} D_{T} \text { and } F^{k} F_{j-1}^{k} B_{k+1}^{-(j+1)} D_{U} .
$$

Since $F^{k} B_{k+1}^{-(\jmath+1)} D_{U}$ and $F^{k} F_{j-1}^{k} B_{k+1}^{-(\jmath+1)} D_{T}$ are neighbors, our assumption leads to three subcases:

First subcase:

$$
F^{k} B_{k+1}^{-(\jmath+1)} \overline{D_{T}} \cap F^{k} F_{j-1}^{k} B_{k+1}^{-(j+1)} \overline{D_{T}} \neq \emptyset .
$$

This leads to the relation

$$
B_{k+j+1}^{-1} \overline{D_{T}} \cap U_{k+j} B_{k+j+1}^{-1} \overline{D_{T}} \neq \emptyset,
$$

which contradicts the second disjointness assumption. 
Second subcase:

$$
F^{k} B_{k+1}^{-(j+1)} \overline{D_{T}} \cap F^{k} F_{j-1}^{k} B_{k+1}^{-(j+1)} \overline{D_{U}} \neq \emptyset .
$$

This leads to the relation

$$
B_{k+j+1}^{-1} \overline{D_{T}} \cap U_{k+j} B_{k+\jmath+1}^{-1} \overline{D_{U}} \neq \emptyset,
$$

which contradicts the third disjointness assumption.

Third subcase:

$$
F^{k} B_{k+1}^{-(j+1)} \overline{D_{U}} \cap F^{k} F_{j-1}^{k} B_{k+1}^{-(\jmath+1)} \overline{D_{U}} \neq \emptyset .
$$

This leads to the relation

$$
B_{k+j+1}^{-1} \overline{D_{U}} \cap U_{k+\jmath} B_{k+\jmath+1}^{-1} \overline{D_{U}} \neq \emptyset,
$$

which contradicts the fourth disjointness assumption.

Apart from the above modifications, all the proofs of Sect. 7 are valid in the case of asymptotic self-similarity.

Now we repeat the construction of the necklace set, using the natural parametrization $t \mapsto \phi(t)$ instead of just working with point sets. The notation is the same as in Sect. 5: we have

$$
\left(U_{0}, T_{0}\right)=B\left(F_{m-1}, F_{m}\right) B^{-1},
$$

where $F_{\jmath}$ denotes the $j^{\text {th }}$ Fibonacci string of the symbols $(R, F)$, and where $B$ is an $m$-fold product of linear-diagonal single-step rescalings. Let $\phi^{0}$ denote the natural parametrization of the invariant pair for $\left(U_{0}, T_{0}\right)$ :

$$
\begin{gathered}
U_{0} \phi^{0}(t)=\phi^{0}(t-1) \quad \text { for } \quad 1-\omega \leq t \leq 1, \\
T_{0} \phi^{0}(t)=\phi^{0}(t+\omega) \text { for } \quad-\omega \leq t \leq 1-\omega .
\end{gathered}
$$

Again, we put

$$
(U, T)=B^{-1}\left(U_{0}, T_{0}\right) B=\left(F_{m-1}, F_{m}\right) .
$$

The maps $U$ and $T$ are commuting homeomorphisms of the whole plane, which means in particular that we do not have to worry about their domains of definition.

Putting $\phi=B^{-1} \phi_{0}$, we obtain

$$
\begin{gathered}
U \phi(t)=\phi(t-1) \quad \text { for } \quad 1-\omega \leq t \leq 1, \\
T \phi(t)=\phi(t+\omega) \quad \text { for } \quad-\omega \leq t \leq 1-\omega .
\end{gathered}
$$

With the definitions

$$
N=\phi([-\omega, 1-\omega]), \quad M=\phi([1-\omega, 1]), \quad L=\phi([-\omega, 1]),
$$

the pair $(M, N)$ is invariant for $(U, T)$, and $\phi$ is a homeomorphism from $[-\omega, 1]$ onto $L=M \cup N$.

Recall from Sect. 5 the recursive definition of the pairs $\left(U_{\jmath}, T_{\jmath}\right)_{\jmath=0}^{m}$ : we put $U_{0}=U$ and $T_{0}=T$ and

$$
U_{\jmath+1}=T_{\jmath} U_{\jmath}^{-1}, \quad T_{j+1}=U_{\jmath}
$$

for $0 \leq j<m$. It follows that $\left(U_{\jmath}, T_{j}\right)=\left(F_{m-\jmath-1}, F_{m-j}\right)$ for $0 \leq j \leq m$ (see Lemma 5.3). ( $U_{j}$ and $T_{j}$ have a different meaning that in the first part of this section.) 
In addition to this, we consider the following recursion: we put $\phi_{0}=\phi$ and define the sequence $\left(\phi_{j}\right)_{j=0}^{m}$ by

$$
\phi_{j+1}(t)= \begin{cases}\phi_{\jmath}\left(-\frac{t}{\omega}\right), & \text { for }-\omega \leq t \leq 1-\omega \\ U_{j} \phi_{j}\left(-\frac{t}{\omega}+1\right) & \text { for } 1-\omega<t \leq 1 .\end{cases}
$$

The following implications hold

$$
\begin{gathered}
-\omega \leq t \leq 1-\omega \Rightarrow-\omega \leq-\frac{t}{\omega} \leq 1, \\
1-\omega<t \leq 1 \Rightarrow-\omega \leq-\frac{t}{\omega}+1<1-\omega .
\end{gathered}
$$

(We have $1-\omega=\omega^{2}$ and $-\frac{1}{\omega}+1=-\omega$ by Definition 6.1.). Therefore, $\phi_{j+1}$ is well defined.

Lemma 8.5. The functions $\phi_{j}$ are continuous, and we have

$$
\begin{gathered}
U_{j} \phi_{j}(t)=\phi_{j}(t-1) \quad \text { for } \quad 1-\omega \leq t \leq 1, \\
T_{j} \phi_{j}(t)=\phi_{j}(t+\omega) \text { for } \quad-\omega \leq t \leq 1-\omega .
\end{gathered}
$$

Proof. For $j=0$, the claim is true by definition. Let $j$ be greater than or equal to zero and assume that the claims have been proved up to $j$.

We first prove that $\phi_{j+1}$ is continuous. By the inductive assumption, it is enough to show that $\phi_{j+1}$ is continuous at $t=1-\omega$. For the limit from below, we obtain

$$
\lim _{t \uparrow 1-\omega} \phi_{j+1}(t)=\phi_{j}\left(-\frac{1-\omega}{\omega}\right)=\phi_{j}(-\omega) .
$$

For the limit from above, we obtain

$$
\lim _{t \downarrow 1-\omega} \phi_{\jmath+1}(t)=U_{\jmath} \phi_{j}\left(-\frac{1-\omega}{\omega}+1\right)=U_{\jmath} \phi_{\jmath}(-\omega+1)=\phi_{\jmath}(-\omega),
$$

where we have used the inductive assumption in the last step, and we see that these limits are equal.

Now we are going to prove the relation $U_{j+1} \phi_{j+1}(t)=\phi_{j+1}(t-1)$. Assume that $1-\omega<t \leq 1$. (Notice the strict inequality.) By definition, we have

$$
U_{j+1} \phi_{j+1}(t)=T_{j} U_{j}^{-1} U_{j} \phi_{j}\left(-\frac{t}{\omega}+1\right)=T_{j} \phi_{\jmath}\left(-\frac{t}{\omega}+1\right) .
$$

The assumption implies the following relations:

$$
-\omega \leq-\frac{t}{\omega}+1<1-\omega, \quad-\omega<t-1 \leq 0<1-\omega .
$$

Using these and the inductive assumption, we obtain

$$
\begin{aligned}
T_{j} \phi_{j}\left(-\frac{t}{\omega}+1\right) & =\phi_{j}\left(-\frac{t}{\omega}+1+\omega\right)=\phi_{j}\left(-\frac{t}{\omega}+\frac{1}{\omega}\right) \\
& =\phi_{j}\left(-\frac{t-1}{\omega}\right)=\phi_{j+1}(t-1)
\end{aligned}
$$


which proves $U_{j+1} \phi_{j+1}(t)=\phi_{j+1}(t-1)$ for $1-\omega<t \leq 1$. By continuity, this holds for $t=1-\omega$ as well.

For the proof of $T_{j+1} \phi_{j+1}(t)=\phi_{j+1}(t+\omega)$, assume that $-\omega \leq t \leq 1-\omega$. By definition, we have

$$
T_{j+1} \phi_{j+1}(t)=U_{j} \phi_{j}\left(-\frac{t}{\omega}\right)
$$

Here, we distinguish two cases:

First case: $1-\omega \leq-\frac{t}{\omega} \leq 1$. In this case, we have the following relations:

$$
\begin{gathered}
\omega^{2}=1-\omega \leq-\frac{t}{\omega} \leq 1, \quad-\omega \leq t \leq-\omega^{3}, \\
-\omega<0 \leq t+\omega \leq-\omega^{3}+\omega=\omega\left(-\omega^{2}+1\right)=\omega^{2}=1-\omega .
\end{gathered}
$$

Making use of the first one, we obtain

$$
U_{\jmath} \phi_{\jmath}\left(-\frac{t}{\omega}\right)=\phi_{j}\left(-\frac{t}{\omega}-1\right)=\phi_{j}\left(-\frac{1}{\omega}(t+\omega)\right) .
$$

Making use of the last one, we obtain

$$
\phi_{j}\left(-\frac{1}{\omega}(t+\omega)\right)=\phi_{j+1}(t+\omega)
$$

by the definition of $\phi_{j+1}$.

Second case: $-\omega \leq-\frac{t}{\omega}<1-\omega$. Let us first rewrite $U_{\jmath} \phi_{j}\left(-\frac{t}{\omega}\right)$ :

$$
U_{\jmath} \phi_{\jmath}\left(-\frac{t}{\omega}\right)=U_{\jmath} \phi_{\jmath}\left(\left(-\frac{t}{\omega}-1\right)+1\right)=U_{j} \phi_{j}\left(-\frac{t+\omega}{\omega}+1\right) .
$$

In this case, we have the following relations:

$$
\begin{gathered}
-\omega \leq-\frac{t}{\omega}<1-\omega=\omega^{2}, \quad-\omega^{3}<t \leq \omega^{2}=1-\omega, \\
-\omega^{3}+\omega=1-\omega<t+\omega \leq 1 .
\end{gathered}
$$

Making use of the last one, we obtain

$$
U_{j} \phi_{j}\left(-\frac{t+\omega}{\omega}+1\right)=\phi_{j+1}(t+\omega)
$$

by the definition of $\phi_{j+1}$

For $j=m$, the preceding lemma yields a continuous function $\phi_{m}$ with

$$
\begin{gathered}
R \phi_{m}(t)=\phi_{m}(t-1), \quad \text { for } \quad 1-\omega \leq t \leq 1, \\
F \phi_{m}(t)=\phi_{m}(t+\omega), \quad \text { for } \quad-\omega \leq t \leq 1-\omega .
\end{gathered}
$$

Now, we put

$$
\phi(t)=R^{-[t+\omega]} \phi_{m}(t-[t+\omega]),
$$

where the Gauss brackets are defined by $[s]=\max \{n \in \mathbb{Z} \mid n \leq s\}$. 
Lemma 8.6. The function $\phi$ is continuous, and we have

$$
\phi(t-n)=R^{n} \phi(t)
$$

for all $t \in \mathbb{R}$ and $n \in \mathbb{Z}$.

Proof. It is enough to show that $\phi$ is continuous at the point $-\omega$. For the limit from below, we obtain

$$
\lim _{t \uparrow-\omega} \phi(t)=R \lim _{t \uparrow-\omega} \phi_{m}(t+1)=R \phi_{m}(1-\omega) .
$$

For the limit from above, we obtain

$$
\lim _{t \downarrow-\omega} \phi(t)=\lim _{t \downarrow-\omega} \phi_{m}(t)=\phi_{m}(-\omega) .
$$

Putting $t=1-\omega$ in the equation $R \phi_{m}(t)=\phi_{m}(t-1)$, we see that these limits are equal.

For the proof of the second claim, observe that we have

$$
\begin{aligned}
\phi(t-1) & =R^{-[t-1+\omega]} \phi_{m}(t-1-[t-1+\omega]) \\
& =R R^{-[t+\omega]} \phi_{m}(t-[t+\omega])=R \phi(t)
\end{aligned}
$$

and

$$
\begin{aligned}
\phi(t+1) & =R^{-[t+1+\omega]} \phi_{m}(t+1-[t+1+\omega]) \\
& =R^{-1} R^{-[t+\omega]} \phi_{m}(t-[t+\omega])=R^{-1} \phi(t)
\end{aligned}
$$

by construction.

\section{Lemma 8.7.}

$$
F \phi(t)=\phi(t+\omega), \quad \text { for all } t \in \mathbb{R}
$$

Proof. Take any $t \in \mathbb{R}$ and put $n=[\omega+t]$, which implies $-\omega \leq t-n<1-\omega$. We then obtain

$$
\begin{aligned}
F \phi(t) & =R^{-n} F R^{n} \phi(t)=R^{-n} F \phi(t-n) \\
& =R^{-n} F \phi_{m}(t-n)=R^{-n} \phi_{m}(t+\omega-n) .
\end{aligned}
$$

Here, we have to distinguish two cases:

First case: $0 \leq t+\omega-n<1-\omega$. In this case, we have $[t+2 \omega]=n$. Continuing the above equation, we obtain

$$
R^{-n} \phi_{m}(t+\omega-n)=R^{-[t+2 \omega]} \phi_{m}(t+\omega-[t+2 \omega])=\phi(t+\omega)
$$

by definition.

Second case: $1-\omega \leq t+\omega-n<1$. In this case, we have $[t+2 \omega]=n+1$. Continuing the above equation, we obtain

$$
\begin{aligned}
R^{-n} \phi_{m}(t+\omega-n) & =R^{-(n+1)} R \phi_{m}(t+\omega-(n+1)+1) \\
& =R^{-(n+1)} \phi_{m}(t+\omega-(n+1)) \\
& =R^{-[t+2 \omega]} \phi_{m}(t+\omega-[t+2 \omega])=\phi(t+\omega)
\end{aligned}
$$

by definition.

Now we are going to show that $\phi$ is actually a homeomorphism. As a preparation, we prove the following two results: 
Lemma 8.8. Let $a$ and $s$ be arbitrary real numbers, and assume that

$$
\phi(s+a)=\phi(s) .
$$

Then we have

$$
\phi(t+a) \equiv \phi(t)
$$

Proof. The assumption implies $R^{p} \phi(s+a)=R^{p} \phi(s)$ for all integers $p$, which is equivalent to

$$
\phi(s-p+a)=\phi(s-p)
$$

by Lemma 8.6. In the same way, the assumption implies $F^{q} \phi(s+a)=F^{q} \phi(s)$ for all integers $q$, which is equivalent to

$$
\phi(s+q \omega+a)=\phi(s+q \omega)
$$

by Lemma 8.7. Combining these two equations, we have

$$
\phi(s+q \omega-p+a)=\phi(s+q \omega-p)
$$

for all pairs $(p, q)$ in $\mathbb{Z}^{2}$. Since the numbers of the form $q \omega-p$ are dense in $\mathbb{R}$, the claim follows by observing that $\phi$ is continuous (see Lemma 8.6).

Lemma 8.9. There exists a positive constant $M$ such that the following implication holds:

$$
|s-t|>M \Rightarrow|\phi(s)-\phi(t)|>1 \text {. }
$$

Proof. The claim follows from the definition of $\phi$, by observing that $\phi_{m}$ is bounded on $[-\omega, 1-\omega]$.

Lemma 8.10. The function $\phi$ is a homeomorphism.

Proof. Since $\phi$ is continuous by Lemma 8.6, it suffices to show that $\phi$ is injective. Assume to the contrary that there exists a real number a different from zero and an arbitrary real number $s$ such that

$$
\phi(s+a)=\phi(s) .
$$

By Lemma 8.8 , we have $\phi(t+a) \equiv \phi(t)$, which implies that

$$
\phi(s+n a)=\phi(s)
$$

for all integers $n$. Let $M$ be the number defined in Lemma 8.9. We choose $n$ such that $|n a|>M$. Using Lemma 8.9, we obtain the contradiction

$$
0=|\phi(s+n a)-\phi(s)|>1 \text {. }
$$

We conclude by observing that $\phi$ is piecewise Hölder continuous by construction, and therefore globally Hölder continuous.

Note added in proof. The proof of Lemma 8.10 does not imply that the map $\phi$, regarded as a map of $\mathbb{R} / \mathbb{Z}$ into the cylinder, is a homeomorphism into its image. The proof of this fact is left to the reader. 


\section{References}

1. Herman, M.R.: Sur les courbes invariantes par les difféomorphismes de l'anneau. Astérisque 1, 103-104 (1983)

2. MacKay, R.S.: Renormalisation in area-preserving maps. Ph.D thesis, Princeton (Univ Microfilms Int, Ann Arbor, Michigan 1982

3. MacKay, R.S.: A renormalisation approach to the breakup of invariant circles in area preserving maps. Physica D 7, 283-300 (1983)

4. Mather, J.N.: Non-existence of invariant circles. Ergod. Th. \& Dynam. Sys. 4, 301-309 (1984)

5. Shenker, S.J., Kadanoff, L.P.: Critical behavior of a KAM surface: empirical results. J. Stat. Phys. 27, 631-656 (1982)

6. Stirnemann, A.: Renormalization for golden circles. Diss. ETH 9843 (1992)

Communicated by J.-P. Eckmann 
